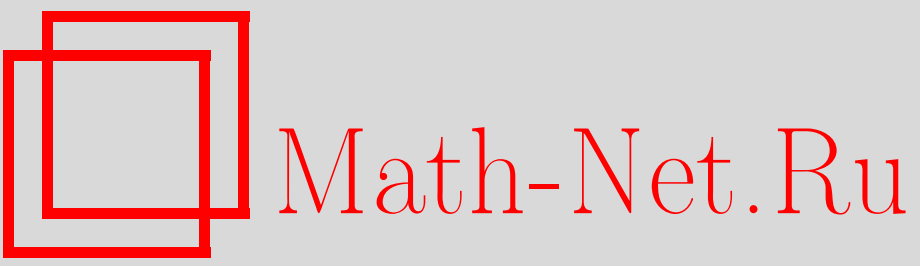

Л. О. Чехов, Матричные модели: Геометрия пространств модулей и точные решения, ТМФ, 2001, том 127, номер 2, 179-252

DOI: https://doi.org/10.4213/tmf455

Использование Общероссийского математического портала Math-Net.Ru подразумевает, что вы прочитали и согласны с пользовательским соглашением

http://www . mathnet.ru/rus/agreement

Параметры загрузки:

IP : 3.85 .5 .30

26 апреля 2023 г., 13:27:59 


\section{МАТРИЧНЫЕ МОДЕЛИ: ГЕОМЕТРИЯ ПРОСТРАНСТВ МОДУЛЕЙ И ТОЧНЫЕ РЕШЕНИЯ ${ }^{1)}$}

Прослежена связь между характеристиками пространств модулей римановых поверхностей с отмеченными точками и матричных моделей. Матричная модель Концевича задает индексы пересечений на непрерывных пространствах модулей, а матричная модель Концевича-Пеннера - на дискретизованных пространствах модулей. На основе анализа алгебр связей, которым удовлетворяют различные матричные модели типа обобщенных моделей Концевича, выведены преобразования времен, устанавливающие точные соотношения между различными моделями, находящими применение в математической физике. Приведено решение эрмитовой одноматричной модели в разложении по родам в технике моментов, и предложена рекуррентная процедура решения этой модели в двойном скейлинговом пределе.

\section{СОДЕРЖАНИЕ}

1. Введение . . . . . . . . . . . . . . . . . . . . . . . . . . . . . . 180

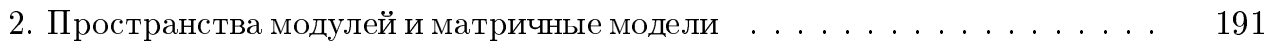

2.1. Непрерывное пространство модулей $\mathcal{M}_{g, n}$. . . . . . . . . . . . . . . . 191

2.2. Дискретизованное пространство модулей $\overline{\mathcal{M}}_{g, n}^{\text {disc }} \ldots \ldots$. . . . . . . . . . . . . . . 198

3. Уравнения связей в матричных моделях . . . . . . . . . . . . . . . . . . . . . 204

3.1. Алгебра Вирасоро в 1ММ ... . . . . . . . . . . . . . . . 204

3.2. Уравнения Швингера-Дайсона и алгебра Вирасоро в матричной модели Концевича . . . . . . . . . . . . . . . . . . . . . . . . . . 206

3.3. Уравнения Швингера-Дайсона для ММКП . . . . . . . . . . . . . . . 209

3.4. Эквивалентность ММКП и 1ММ . . . . . . . . . . . . . . . . . . . . 210

4. Вычисления в матричных моделях в разложении по родам . . . . . . . . . 214

4.1. Петлевые средние в 1 ММ . . . . . . . . . . . . . . . . . . . . . 214

4.2. Процедура итераций . . . . . . . . . . . . . . . . . . . . . . . 217

4.3. Двойной скейлинговый предел . . . . . . . . . . . . . . . . . . . 222

1) Статья написана по заказу Редколлегии.

* Математический институт им. В. А. Стеклова РАН, Москва, Россия. E-mail: chekhov@mi.ras.ru 
5. Обобшенные модели Концевича с неполиномиальными потенциалами . . 231

5.1. Матричная модель суперструны IIВ . . . . . . . . . . . . . . . . . . . . 231

5.2. Двухлогарифмическая матричная модель с внешним полем . . . . . 236

6. Точные соотношения между матричными интегралами Концевича-Пеннера и Концевича . . . . . . . . . . . . . . . . . . . . . . . . . . . . . . . . 243

6.1. Соотношение между моментами и переменными ДПМ ...... 243

6.2. Алгебра времен $t_{2 n}^{ \pm} \ldots \ldots \ldots \ldots \ldots$. . . . . . . . . . . . . . . . . . . .

6.3. Уравнения Швингера-Дайсона в терминах времен $t_{2 n}^{ \pm} \ldots \ldots . . . .245$

6.4. Алгебра связей $L_{2 s+1}^{ \pm}$. . . . . . . . . . . . . . . . . . . . . . . 247

\section{1. ВВЕДЕНИЕ}

Основной мотив, побуждаюший к исследованию матричных моделей, - это развитие непертурбативных методов в теории струн. Струнная теория допускает целую серию пертурбативных вакуумов, описываемых двумерными конформными теориями поля (КТП). Для того чтобы описывать переходы между этими вакуумами и конечные флуктуации струнной теории, необходимо отказаться от описания теории на уравнениях движения и рассмотреть континуальньй интеграл по всем двумерным метрикам. Наиболее успешный подход к непертурбативной струнной теории - это так называемая теория струн в некритических размерностях, задаваемая тремя эквивалентными описаниями в терминах двумерной гравитации, двумерных топологических теорий и матричных моделей, которые в тех случаях, когда можно сравнить результаты, полученные различными методами, оказывались в полном согласии друг с другом. При этом именно с помошью матричных моделей оказалось возможным провести вычисления, которые слишком сложно или даже невозможно проделать в других подходах.

Начало теории некритических струн было положено в работах Полякова [1], в которых интеграл, зависящий от нелинейного действия Намбу-Гото, был линеаризован с помощью введения дополнительного континуального интегрирования по пространству двумерных метрик. Два описания совпадают на уравнениях движения; принципиальное отличие состоит в том, что в подходе Полякова струнные переменные (координаты пространства-времени) трактуются как (классические либо квантовые) поля на двумерной поверхности. Калибровочная инвариантность приводит к тому, что поляковское действие на классическом уровне не зависит от метрики. Квантовые поправки вводят конформную (вейлевскую) аномалию, и гравитационные моды приобретают нетривиальную динамику, задаваемую двумерным действием Лиувилля. В критической размерности ( $D=26$ для бозонной и $D=10$ для фермионной струны) конформная аномалия исчезает и поля остаются свободными. Сначала считалось, что именно по этой причине теория некритических струн непригодна для согласованного описания какой-либо струнной теории. Но именно в теории некритических струн оказалось возможным за- 
дать механизм динамического возникновения пространства-времени Минковского, в котором поле Лиувилля можно интерпретировать как переменную времени [2].

Тем не менее нахождение решений в теории некритических струн именно из-за возникающего на квантовом уровне взаимодействия представляет собой исключительно сложную проблему, первым шагом к решению которой были найденные в работах [3]-[5] критические индексы теории. В этих работах авторы рассматривали двумерные КТП, присоединенные к гравитации. Существенный параметр КТП - это центральный заряд $c$ (равный количеству струнных полей поляковской струны). В подходе Полякова размерность пространства-времени равна $c+1$. Если эта размерность лежит между 2 и 26, то действие двумерной гравитации становится комплексным, т.е. теория содержит комплексные аномальные размерности и вакуум теории становится нестабильнымструна переходит в полимерную фазу [6].

Основной проблемой квантовой теории поляковской струны остается вопрос о правильном определении меры интегрирования в интеграле по метрикам двумерной гравитации. В решении этой проблемы как раз и помогают матричные модели. Первичная мотивация использования матричных моделей сводится к нахождению теорий, проявляющих свойства физической гравитации. Важнейшее среди этих свойств - это топологическая природа струнного действия [7]. В случае, когда струнная теория не содержит выделенной метрики, корреляционные функции теории суть числа, зависящие лишь от (дискретного) набора полей и топологических характеристик (род, число вставок полей либо выколотых точек) двумерной поверхности, в то время как ввиду общей ковариантности зависимости от координат оставаться не должно.

Описание струнной теории с помошью двумерных топологических теорий было предложено Виттеном [7], [8], рассмотревшим теорию, коллективные переменные которой суть координаты пространства модулей римановых поверхностей (РП), а корреляторы задаются интегрированием по пространствам модулей от геометрически локально-инвариантных объектов - произведений замкнутых 2-форм. Эта конструкция использовала изошренную процедуру двойного скейлингового предела (ДСП) эрмитовой одноматричной модели (соответствующей изначально чистой гравитации без полей материи) и потому не годилась для описания присоединенной материи. Тем не менее подход Виттена оказался весьма плодотворным - на его основе возникло множество других матричных теорий (матричная модель Концевича [9] и ее обобщения), оказавшихся полезными как в физике, так и в математике. Матричные модели не только воспроизводят все известные результаты, полученные в рамках топологической или непосредственно физической гравитации, они позволяют решить модель точно, т.е. найти произвольную коррелящионную функцию на РП произвольного рода.

В настояшей работе исследуются матричные модели, описываюшие топологические двумерные теории. Основной задачей будет построение техники вычислений, позволяющей получать явные ответы в разложении по родам (соответствующем $(1 / N)$-разложению 'т Хоофта [10]). В связи с этим, с одной стороны, затрагиваются вопросы связи матричных моделей с КТП и с точно интегрируемыми системами с помощью механизма $\tau$-функции и процедуры ДСП, а с другой стороны, описано, как матричные модели спо- 
собствовали использованию геометрических идей в вычислениях физических величин корреляторов в теориях двумерной гравитации.

Обзоры по теории матричных моделей можно найти в работах [11], [12] и в классической книге Мехты [13]. Необходимо также отметить книгу [14], в которой обсуждаются точные решения петлевых уравнений и техника моментов ${ }^{2}$.

Первоначальный интерес к матричным моделям был связан с тем, что эти модели рассматривались как "хорошая" дискретизация двумерных поверхностей, различающая поверхности различного рода и позволяющая, например, вычислять число таких различных триангулированных поверхностей при фиксированном числе плакеток (треугольников) и для фиксированного рода. Если считать, что такое разбиение в пределе большого числа плакеток стремится к правильной мере в струнном поляковском интеграле по пространству модулей [1], то этот интеграл оказывается хорошо определенным: в то время как число всех графов с заданным числом плакеток растет факториально и не имеет предела ни при какой константе связи, это же число в каждом отдельном роде имеет экспоненциальньй рост, который может быть скомпенсирован перерастяжкой константы связи (простейший пример - процедура ДСП). Заметим, что при этом рассматривались и решались, как правило, одноматричные модели эрмитовых матрищ с полиномиальными потенциалами не слишком высокого (третьего или четвертого) порядка; считалось, что рассматривать потенциалы более высоких порядков хотя и возможно, но технически сложно, а главное, не нужно, так как для воспроизведения эффектов струнной меры достаточно классических решений [15].

Дальнейшее развитие КТП, теории некритических струн и интегрируемых моделей привело к идее использования матричных моделей более сложного вида (для начала одноматричных моделей с более сложными потенциалами) для описания критических явлений в теориях двумерной гравитации с материей. Основной объект исследования матричных моделей - многопетлевой коррелятор вида

$$
W\left(p_{1}, \ldots, p_{n}\right)=\left\langle\operatorname{tr} \frac{1}{p_{1}-Y} \ldots \operatorname{tr} \frac{1}{p_{n}-Y}\right\rangle
$$

где степени величин $p_{i}$ отвечают вставкам “петель" определенной длины в терминах матрицы интегрирования $Y$, но ни взаимное расположение петель, ни даже их возможные пересечения не фиксированы; единственная дополнительная зависимость связана с родом поверхности $g$. При этом, поскольку все результаты, получаемые в рамках матричных моделей, оказываются не зависящими от координат, то речь идет о типичных топологических теориях. Было обнаружено, что выбирая матричные модели с потенциалами старших порядков и применяя процедуру ДСП [16]-[18], при которой “ведущие” вклады, характерные для чистой гравитации, сокрашаются, можно получить корреляторы со старшими критическими индексами (аномальными размерностями).

\footnotetext{
2) Авторы этой книги не упоминают (намеренно) термин матричные модели, однако основные объекты исследования - петлевые уравнения - исторически возникли именно из матричных интегралов.
} 
Одновременно с этим возникло понимание того, что интегралы матричных моделей, выраженные через соответствующие времена, подчиняются уравнениям интегрируемых иерархий (более точно, эти интегралы суть $\tau$-функции иерархий Кадомцева-Петвиашвили для интегралов с внешним полем, иерархий полубесконечной цепочки Тоды для одноматричных моделей и т.д., см. [19], [20] и ссылки в этих работах). Виттен [21] выдвинул гипотезу, что ДСП одноматричной модели, задающий производящую функцию для корреляторов двумерной топологической гравитации с материей, подчиняется уравнениям Кортевега-де Фриза (Кд $\Phi)$. В развитие этой гипотезы был открыт новый тип матричных моделей, непосредственно описываюший эту предельную теорию, заданную на пространстве модулей комплексных кривых.

Поскольку в подходе триангуляций комплексная структура РП не играет роли, этот подход можно использовать и для описания других теорий, не обязательно связанных со струнами. В самом деле, имеются матричные реализации редуцированных теорий Янга-Миллса через матричные модели в произвольной размерности (см. [22] и приведенные там ссылки), и даже предлагается представить гипотетическую одиннадцатимерную М-теорию, которая, возможно, объединяет все струнные теории, через матричный интеграл [23], [24].

Помимо интегрируемости есть еше одно свойство, характерное для всех матричных моделей. Во всех этих моделях возникают алгебры связей, которым удовлетворяет статистическая сумма матричной модели. В данной работе рассматриваются матричные модели, удовлетворяюшие условиям связи, подчиненным алгебрам Вирасоро. Сами эти условия могут рассматриваться как определяющие, в то время как матричный интеграл оказывается всего лишь некоторым (иногда вполне неоднозначным) представлением решений соответствуюших уравнений. Возникновение алгебр Вирасоро отражает топологическую природу матричных моделей, а важное свойство интегрируемости кажется более скрытым.

Интегрируемые деформации КТП известны достаточно давно [25]. Кроме того, в струнном подходе ожидалось, что и статистическая сумма струны может задаваться (классической) интегрируемой системой [26], [27]. В струне интегрируемые системы возникают, если проинтегрировать некоторые $\tau$-функции [28], [29] по универсальному пространству модулей [30]. Возникающие квантовые системы разделяются на два класса в зависимости от того, задаются ли классические интегрируемые системы временами (константами связи) или переменными Мивы, построенными из собственных значений внешней бесконечной (в пределе $N \rightarrow \infty$ ) матрицы. Оба варианта реализуются в матричных моделях: статистическая сумма дискретной матричной модели - это $\tau$-функция, времена которой - константы связи, в то время как статистическая сумма непрерывной матричной модели - это $\tau$-функция, зависяшая от внешней матрищы, задающей переменные Мивы. Как будет показано ниже, оба эти случая взаимосвязаны и являются частными случаями непрерывных матричных моделей с не обязательно полиномиальными потенциалами.

$\tau$-Функция допускает множество решений. Инвариантное условие, выделяюшеенужное решение, - это струнное уравнение. После наложения этого единственного допол- 
нительного условия матричный интеграл становится однозначно определенным и возникает полубесконечная алгебра связей типа Вирасоро или $W$, накладываемых на этот интеграл [31]. Эта алгебра связей на самом деле есть набор тождеств Уорда для данной модели, отвечающих вариациям констант связи. В то время как в теории поля тождества Уорда не задают динамику полностью, в матричных моделях они эквивалентны уравнениям движения (снова ввиду топологичности теории), т.е. тождества Уорда эквивалентны уравнениям Швингера-Дайсона.

Для описания двумерных топологических теорий были разработаны два матричномодельных подхода.

Первый подход [16]-[18] связан с использованием обычной эрмитовой одноматричной модели с произвольным потенциалом. В технике ориентированных ("ленточных") графов по любому графу можно построить дуальный граф, отвечающий РП с особенностями кривизны, сосредоточенными в вершинах дуального графа. Грани (двумерные симплексы) этого графа отвечают вершинам матрично-модельного графа, и наоборот. Если, например, исходный потенциал содержал только трехвалентные вершины, то дуальньй граф будет состоять из треугольных граней с вершинами произвольного порядка, т.е. получается триангулированная РП. Хотя в дальнейшем будут встречаться потенциалы с вершинами произвольного порядка, разбиения РП, задаваемые такими потенциалами, будут также называться триангуляциями. Матричная модель с произвольным потенциалом была точно решена в работах [16]-[18] в ДСП, в котором преобладающий вклад дают разбиения с числом граней, стремящимся к бесконечности, а сингулярные метрики аппроксимируют “случайную метрику” на РП. Эта модель (в дальнейшем мы обозначаем ее $1 \mathrm{MM}$ ) описывалась эрмитовым одноматричным интегралом по матрицам $Y$ размера $M \times M$ :

$$
\int_{M \times M} \exp (\operatorname{tr} V(Y)) D Y
$$

где

$$
V(Y)=\sum_{n} \xi_{n} \operatorname{tr} Y^{n}
$$

а $\xi_{n}$ называются временами одноматричной модели. Системы такого типа подчиняются уравнениям иерархии дискретной цепочки Тоды с наложенными дополнительными условиями Вирасоро (условие конформной точки) [32]-[37]. В ДСП параметр $M$ стремится к бесконечности, а $V(X)$ преобразуется таким образом, чтобы поверхности, даюшие главный вклад, отвечали разбиениям с неограниченно растушим числом граней. В этом пределе появляются уравнения иерархии Кд $\Phi$, а статистическая сумма двумерной гравитации задается степенным разложением по счетному числу переменных и совпадает с логарифмом некоторой $\tau$-функции иерархии КдФ [37].

Во втором (когомологическом) подходе используется тот факт, что фазовое пространство двумерной гравитации есть пространство модулей комплексных кривых. Описание топологии пространств модулей $\mathcal{M}_{g, n}$ алгебраических кривых рода $g$ с $n$ отмеченными точками (вставками полей материи) с помощью матричных моделей восходит к работам Концевича [9], в которых была построена матричная модель, задаюшая производящую 
функцию индексов пересечений на пространствах модулей $\mathcal{M}_{g, n}$. Эти индексы-геометрические инварианты, которые с физической точки зрения суть не что иное, как корреляционные функции топологической гравитации. Они выражаются через интегралы от первых классов Черна (замкнутых 2-форм) по модулярным (орби)пространствам $\overline{\mathcal{M}}_{g, n}$ :

$$
\left\langle\tau_{d_{1}} \ldots \tau_{d_{n}}\right\rangle_{g}=\int_{\overline{\mathcal{M}}_{g, n}} \prod_{i=1}^{n} \omega_{i}^{d_{i}}
$$

где $\omega_{i}$ - первый класс Черна, ассоциированный с $i$-й отмеченной (проколотой) точкой. Как и должно быть в топологической теории, формула (1.2) не зависит от выбора координат: величины, входящие в нее, представляют собой “инварианты зацеплений" на данных (орби)пространствах. Если бы рассматривались обычные многообразия, допускаюшие всюду гладкую координатизацию, то эти величины были бы неотришательными иельми числами. Орбифолдная природа модулярных пространств означает, что орбипространство $\mathcal{M}_{g, n}$ (или, более точно, его подходящее замыкание $\overline{\mathcal{M}}_{g, n}$ ) может быть получено из накрывающего многообразия $\mathcal{T}_{g, n}$ факторизацией по группе дискретных симметрий конечного порядка $\Gamma_{g, n}: \mathcal{M}_{g, n}=\mathcal{T}_{g, n} / \Gamma_{g, n}$. Стационарные точки этой группы суть особые точки метрики на $\mathcal{M}_{g, n}$. Ввиду такой факторизации соответствующие индексы становятся неотрицательными рациональны.ми числами.

Техника матричных моделей позволяет объединить преимушества этих двух подходов и установить явные соответствия между моделями (1.1) и (1.2).

По гипотезе Виттена [7] индексы пересечений - интегралы от первых классов Черна на соответствующих пространствах модулей - имеют смысл корреляционных функций двумерной гравитации с присоединенной материей. Таким образом, в непрерывном случае должно выполняться соотношение (1.2), при этом интегралы должны браться по правильным образом компактифицированным пространствам модулей, а замкнутые 2-формы $\omega_{i}$ суть представители первых классов Черна линейных расслоений на $\overline{\mathcal{M}}_{g, n}$.

Введение координат на пространствах модулей в униформизации Штребеля, описываемое в разделе 2 , осушествляется в терминах ленточных графов; при этом всем ребрам графа присваиваются положительные числа $l_{i}$, а полное число проколотых точек $n$ есть число граней ориентированного графа. Появление ленточных графов характерно для матричных моделей. Концевич показал, что индексы пересечений в непрерывном случае выражаются через следующий матричный интеграл (матричная модель Концевича $(\mathrm{MMK}))$ :

$$
\int D X \exp \left\{\operatorname{tr} \frac{1}{2} \Lambda X^{2}+\frac{1}{6} X^{3}\right\}
$$

в которьй в качестве параметра входит внешняя (эрмитова) матрица $\Lambda$. Этот интеграл удовлетворяет уравнениям иерархии $\mathrm{K} д \Phi$ по отношению к временам $t_{n}=$ $(2 n-1) ! ! \operatorname{tr} \Lambda^{-2 n-1}[27]$, [38] и задает асимптотическое разложение для струнной статистической суммы

$$
\tau(t)=\exp \sum_{g=0}^{\infty}\left\langle\exp \sum_{n} t_{n} \tau_{n}\right\rangle_{g}
$$


являющейся одновременно $\tau$-функцией иерархии КдФ, взятой в такой точке грассманниана, в которой она инвариантна относительно действия дополнительных связей Виpacopo: $\mathcal{L}_{n} \tau(t)=0, n \geqslant-1[31],[8],[39]-[41]$.

Обобщенная модель Кониевича (ОМK) [42]-[44] связана с иерархией уравнений двумерной решетки Тоды. Эта модель задается интегралом с внешним полем

$$
Z[\Lambda ; N]=\int D X \exp \left\{N \operatorname{tr}\left(\Lambda X-V_{0}(X)\right)\right\}
$$

в котором

$$
V_{0}(X)=\sum_{n=1}^{n_{\max }} t_{n} \operatorname{tr} X^{n}
$$

- потенциал, $t_{n}$ - времена иерархии, интегрирование идет по эрмитовым $(N \times N)$-матрицам. При $V_{0}(X) \sim \operatorname{tr} X^{3}$ получим MМK (1.3). Для вычисления интеграла (1.5) полезно использовать технику уравнений Швингера-Дайсона (ШД) [45], [46], записанных через собственные значения матрицы $\Lambda$.

В терминах времен, или, что равносильно, распределения собственных значений матриц $\Lambda$, уравнения ШД приводят к интегро-дифференциальным уравнениям порядка $n_{\max }-1$ (для полиномиальных потенциалов). В свою очередь, это означает, что алгебра связей, возникающая из уравнений ШД, связана с алгеброй $W_{n_{\max }-1}\left(W_{2}\right.$ для MMK, $W_{3}$ для потенциала четвертого порядка и т.д.). Алгебра $W_{2}$ есть алгебра Вирасоро, она допускает линеаризованные коммутационные соотношения и может быть разрешена в порядках разложения по родам. Модель Концевича была решена в работах [40], [47], [48] для рода ноль (планарные графы) и в работе [38] для старших родов. Связи Вирасоро модели Концевича, $\mathcal{L}_{n} \mathcal{Z}_{\mathrm{K}}\left(\left\{t_{n}\right\}, N\right)$, зависят лишь от нечетных времен $t_{n}=\left(\operatorname{tr} \Lambda^{-2 n-1}\right) / N$. Произвольная ОМК (1.5) удовлетворяет уравнениям иерархии Кадомцева-Петвиашвили. В то же время ОМК с полиномиальными потенциалами $р$-го порядка удовлетворяет $(p-1)$-редуцированным уравнениям, т.е. исчезает зависимость от времен с номерами $n(p-1)$. Так, ММК не зависит от четных времен, при этом иерархия уравнений Кадомцева-Петвиашвили редуцируется до иерархии КдФ.

Следующее направление исследований в матричных моделях было инициировано вопросом: если полученные классические интегралы отвечают корреляционным функциям квантовой теории на непрерывном пространстве-времени, то можно ли каким-либо образом дискретизовать (проквантовать) само пространство-время? В этой связи представляется интересным найти какие-либо деформации структур пространств модулей, которые бы сохраняли свойства интегрируемости и в той или иной мере свойство топологичности. Возможный вид такой деформации подсказывается конструкцией Воеводского и Шабата [49]-[51]. В этой конструкции функции Белого (важные с точки зрения арифметических униформизаций РП) строятся с помошью ленточных графов в униформизации Штребеля, но с дополнительным разбиением ребер путем введения на них конечного числа дополнительных 2-вершин. При этом линейное пространство функций на таких коротких ребрах имеет максимальные симметрии, если положить все длины получившихся (коротких) ребер равными одному и тому же числу (не ограничивая общности, единице). В работах [52], [53] был предложен подход, позволяюший дискретизовать 
пространство модулей произвольной алгебраической кривой. При этом собственно дискретизация формально совпадает с дискретизацией по Воеводскому и Шабату. В разделе 2 устанавливается соответствие между такими дискретизованными пространствами модулей (ДПМ) и матричными моделями и показано, каким образом в пределе малого параметра дискретизации соответствующая матричная модель - так называемая матричная модель Концевича-Пеннера (ММКП), введенная в [54], - переходит в ММК [9]. Термин ММКП обязан своим происхождением тому, что эта модель включает в себя как предельные случаи (совершенно, впрочем, разной природы) две известные матричные модели: ММК и так называемую матричную модель Пеннера (1MМ), задаваемую интегралом

$$
\int D M \exp \{\alpha N \operatorname{tr}(\ln (1+M)-M)\}
$$

Асимптотическое разложение этой модели, исследованной в работах [55]-[57], генерирует “виртуальные эйлеровы характеристики" $\kappa_{g, s}$ пространств модулей проколотых $\mathrm{P \Pi ,}$

$$
\kappa_{g, s}=\sum_{\substack{\text { клетки } \\ \text { (графы) }}} \frac{(-1)^{n_{G}}}{\# \operatorname{Aut} G},
$$

где суммирование ведется по всем клеткам пространства модулей, а симметрийньй фактор \# Aut $G$, совпадающий с объемом группы автоморфизмов соответствующего графа (клетки), показывает, сколько раз данная клетка накрывает соответствующую область пространства модулей. Числа $\kappa_{g, s}$ - положительные рациональные числа, не обязательно целые ввиду орбиффолдной природы пространства модулей. Формула (1.7) замечательна тем, что, во-первых, интеграл (1.6) легко вычисляется явно и, во-вторых, она допускает обобшения на пространства модулей неориентируемых поверхностей (т.е. этот интеграл явно вычисляется в случаях, когда матрица $M$ принадлежит алгебре $O(N)$ [58] или $S p(2 N)$ (см. приложение 2 в работе [59]).

Аналогом дифференциальных структур на ДПМ служат конечно-разностные структуры, а вместо $U(1)$-расслоений, порождающих первые классы Черна в непрерывном случае, в дискретном случае рассматриваются " $\mathbb{Z}_{p}$-расслоения", а интегралы заменяются на поточечные суммирования. Когомологические классы для ДПМ задаются формулой

$$
\left\langle\left\langle\tau_{d_{1}} \ldots \tau_{d_{n}}\right\rangle\right\rangle_{g}=\int_{\tilde{\pi}^{-1}\left(p_{*}\right)} \prod_{i=1}^{n} \widetilde{\omega}_{i}^{d_{i}}
$$

и могут быть отличными от нуля и при $\sum_{i} d_{i}<3 g-3+n$. Связь между пространствами модулей $\overline{\mathcal{M}}_{g, n}$ и ДПМ была найдена с помощью техники матричного интегрирования в работе [52], где было установлено соотношение между ДПМ и ММКП, задаваемой интегралом по эрмитовым $(N \times N)$-матрицам

$$
\begin{gathered}
\mathcal{Z}_{\mathrm{KP}}[\Lambda]=\int D X \exp \left\{\alpha N \operatorname{tr}\left[-\frac{1}{2} \tilde{\Lambda} X \tilde{\Lambda} X+[\ln (1+X)-X]\right]\right\}, \\
\tilde{\Lambda}=\operatorname{diag}\left(e^{\lambda_{1}}, \ldots, e^{\lambda_{N}}\right) .
\end{gathered}
$$


Важную роль при этом играют эффекты редукции пространств модулей, т.е. модули сингулярных поверхностей, лежаших в замыкании $\partial \mathcal{M}_{g, n}=\overline{\mathcal{M}}_{g, n}-\mathcal{M}_{g, n}$ пространства модулей. Два набора времен, соответствующих ДПМ, задаются формулами

$$
T_{k}^{ \pm}=\frac{1}{(k+1) !} \sum_{i=1}^{N} \frac{\partial^{k}}{\partial \lambda_{i}^{k}} \frac{1}{e^{\lambda_{i}} \pm 1}, \quad k=0,1, \ldots
$$

и статистическая сумма модели (1.9) зависит только от четных времен $T_{2 k}^{ \pm}$.

Примечательно, что интеграл (1.9) явно сводится к модели (1.5) с неполиномиальным.м потенциалом $V_{0}(X)=-X^{2} / 2+\alpha N \ln X$ и внешним полем $\Xi=\tilde{\Lambda}+\tilde{\Lambda}^{-1}$. Уравнения ШД этой модели снова имеют вид квадратичных интегро-дифференциальных уравнений, решаемых в рамках разложения по родам [54], [60] (см. раздел 3).

Разработана техника вычислений, позволяющая найти все необходимые характеристики пространств модулей, в разложении по родам, эквивалентном $(1 / N)$-разложению по обратному размеру матрищ в интеграле (разложения такого рода широко известны в теоретической физике начиная с работы 'т Хоофта [10]), в некотором смысле “наиболее простым" способом. Такое представление весьма полезно и для исследования критических свойств соответствуюшей теории. Известно [45], [46], что наиболее явный ответ для разложения по родам матричных моделей достигается в технике моментов. Будем считать матричную модель “точно решаемой”, если ее статистическая сумма может быть представлена в каждом роде (порядке по $1 / N^{2}$ ) как рациональная функция от некоторых новых переменных (моментов), которые содержат одновременно явную линейную зависимость от исходных переменных (времен) и неявную зависимость от тех же времен через конечное (зависящее только от исходной задачи) число вспомогательных переменных, определяемых из нелинейных алгебраических соотношений.

По-видимому, список матричных моделей с внешним полем, допускающих рациональные решения в терминах моментов, исчерпывается моделями, для которых уравнения ШД приводятся к уравнениям второго порядка и в конечном итоге к условиям Вирасоро. Для всех таких моделей были найдены соответствующие пертурбативные разложения. Исторически первой (и наиболее простой из решенных) моделью была матричная модель Брезана-Гросса-Виттена [45], [61]

$$
\int D U \exp \left(J^{\dagger} U+U^{\dagger} J\right)
$$

с унитарными матрицами интегрирования $U, U^{\dagger} \in U(N)$ и внешними матрицами $J^{\dagger}, J$. После замены переменных интегрирования $U=e^{X}$ модель (1.11) приводится к эрмитовой матричной модели

$$
\mathcal{Z}_{\mathrm{BGW}}=\frac{1}{V_{N}} \int D X \exp \left\{\operatorname{tr}\left(J^{\dagger} X-N \ln X+X^{-1} J\right)\right\}
$$

с неполиномиальным потенциалом, зависящим от внешнего эрмитова матричного поля $M=J^{\dagger} J$. 
MMK (1.3) была решена в терминах моментов в работе [38], где вместо использования условий Вирасоро строилось разложение моментов решения иерархии уравнений Кд $\Phi$, пополненных струнным уравнением. При этом, как показано в п. 4.3.3, сама модель и ее условия связи могут быть переформулированы в терминах моментов, принимаемых за новые времена. Рекуррентная процедура при этом сильно упрошается и многие важнейшие непертурбативные характеристики могут быть явно вычислены для любого рода. Наконец, была введена и решена ММКП (1.9) для родов ноль [54] и один [62], и была показана эквивалентность этой модели и $1 \mathrm{MM}$ [62] с произвольным потенциалом, константы связи в котором связаны с внешним полем $\Xi$ преобразованием Концевича-Мивы

$$
\xi_{n}=\frac{1}{n} \operatorname{tr} \Xi^{-n}-\frac{\alpha N}{4} \delta_{n, 2}, \quad n \geqslant 1 .
$$

Это позволило построить итеративную процедуру для нахождения решений $1 \mathrm{MM} \mathrm{c} \mathrm{про-}$ извольным потенциалом в разложении по родам [53], обсуждаемую в разделе 4. Общая структура вклада рода $g$ имеет вид

$$
F_{g}=\sum_{\substack{\alpha_{j}>1 \\ \beta_{i}>1}}\left\langle\alpha_{1} \ldots \alpha_{s} ; \beta_{1} \ldots \beta_{l} \mid \alpha, \beta, \gamma\right\rangle_{g} \frac{M_{\alpha_{1}} \ldots M_{\alpha_{s}} J_{\beta_{1}} \ldots J_{\beta_{l}}}{M_{1}^{\alpha} J_{1}^{\beta} d^{\gamma}}, \quad g \geqslant 1 .
$$

Эта сумма конечна для каждого рода, а два набора моментов естественным образом ассоциируются с двумя наборами времен ДПМ. При этом в ДСП половина моментов $1 \mathrm{MM}$ переходит в соответствующие моменты MMK, а другая половина просто не дает вклада. Кроме того, сама процедура ДСП, задаваемая довольно сложным преобразованием на уровне интеграла $1 \mathrm{MM}$, как оказывается, получается простой растяжкой полей в интеграле (1.9).

В самое последнее время интерес к матричным моделям вновь оживился в связи с матрично-модельной формулировкой М-теории [23], которая, как предполагается, описывает непертурбативный подход в теории суперструн. Соответствующая матричная модель [23], по-видимому, дает полное квантово-механическое описание М-теории. Техника этого подхода обсуждается в разделе 5 , здесь же отметим, что в нем естественным образом возникает матричный интеграл вида (1.12), который описывает усреднение по гравитационным степеням свободы. Соответствуюшее нелинейное действие Борна-Инфельда матричной модели суперструны типа IIB [63], [64]

$$
S_{\mathrm{NBI}}=-\frac{\alpha}{4} \operatorname{tr} Y^{-1}\left[A_{\mu}, A_{\nu}\right]^{2}+\beta \operatorname{tr} Y+2 \eta \operatorname{tr} \ln Y-\frac{1}{2} \operatorname{tr} \bar{\Psi} \Gamma^{\mu}\left[A_{\mu}, \Psi\right]
$$

обладает двумерной суперсимметрией в пределе больших $N$ [63]. Это описание - еше одна (квази)линеаризация существенно нелинейного струнного действия Намбу-Гото, в которой матрица $Y$ играет роль метрики мирового листа, интегрирование по которой дает эффективное действие. Стандартное неабелево действие Борна-Инфельда получается при $\eta=-1 / 4 N$, где $N$ - размер матриц. Тем не менее теории с другими значениями $\eta$ рассматривались ранее [65], а ответ для эффективного действия в ведушем порядке по $N$ был получен в работе [66] для случая константы $\eta$ общего положения. 
Матричная модель струны IIB (обобшение матричной модели Брезана-Гросса-Виттена на случай произвольной константы перед логарифмическим членом в формуле $(1.12))[66],[67]$

$$
\begin{gathered}
Z_{\mathrm{IIB}}=\int D X \exp \left\{-N \operatorname{tr}\left[X \Lambda+X^{-1}+(2 \eta+1) \ln X\right]\right\}, \\
t_{k}=\frac{1}{2 k-1} \operatorname{tr} \Lambda^{-k+\frac{1}{2}}, \quad k=1,2, \ldots,
\end{gathered}
$$

и матричная модель с двумя логарифмическими членами (2-lnMM) [68]

$$
Z_{2-\ln }=\int D X \exp \{-N \operatorname{tr}[X \Lambda+\alpha \ln (1-X)+\beta \ln (1+X)]\}
$$

были решены в терминах моментов. Одновременно оказалось, что решения моделей (1.15) и (1.16) могут быть получены с помощью достаточно простого преобразования времен (и моментов) вида

$$
\tilde{t}_{k}=t_{k}-\frac{2 \eta N}{2 k+1} \frac{1}{\left(\eta+\frac{1}{2}\right)^{2 k+1}}, \quad k \geqslant 3, \quad \tilde{t}_{1,2}=t_{1,2}
$$

из соответствующих решений ММК [67] и ММКП [68]. (Вообще, как показывает практика, именно условия связи типа условий Вирасоро позволяют устанавливать наиболее нетривиальные соотношения между различными моделями.) Заметим, что преобразования времен типа (1.17) хотя и близки к преобразованиям Концевича-Мивы (1.13), но не сводятся к ним непосредственно ввиду специальных сдвигов первых двух времен. Соответствуюшее описание непосредственно в терминах $\tau$-функции оказывается достаточно сложным и до сих пор не построено.

Другие матричные модели, допускающие решения в терминах моментов, - это так называемые $O(n)$-модели [69]-[72], возникающие при описании критических явлений в спиновых системах.

Во всех исследуемых матричных моделях фундаментальная роль принадлежит переменным "времен". Заметим, что из результатов разделов 2 и 3 возникает вопрос о "правильном" выборе времен в 1ММ. Например, наличие удвоенного набора моментов 1ММ, выражаемых как через времена (1.13), так и через времена ДПМ (1.10), в отличие от одного набора времен в случае MMK, не позволяет сформулировать 1ММ (или ММКП) только лишь в терминах моментов с использованием уравнений соответствующей интегрируемой иерархии (в данном случае иерархии цепочки Тоды). В то же время цепочка Тоды допускает формулировку, включающую в себя некоторое "прямое произведение" $\tau$-функций КдФ (с различнылми временами), связанное через дискретное время $M$ - размер матриц [73]. Как показано в разделе 6, точно такое же разбиение возможно в случае ММКП, однако показать его пока удалось только на уровне уравнений связи - с помощью исследования генераторов Вирасоро, соответствующих вариациям времен (1.10). В результате некоторого явно найденного в разделе 6 канонического преобразования статистическая сумма ММКП преврашается в произведение двух 
статистических сумм MМK, взятых при различных временах и сплетенных оператором канонического преобразования $\mathcal{A}$ :

$$
\mathcal{Z}_{\mathrm{KP}}\left(\left\{T_{n}^{ \pm}\right\}, \alpha N\right)=e^{C(\alpha N)} e^{\mathcal{A}}\left(\mathcal{Z}_{\mathrm{K}}\left(\left\{T_{n}^{+}\right\}, \alpha N\right) \cdot \mathcal{Z}_{\mathrm{K}}\left(\left\{T_{n}^{-}\right\}, \alpha N\right)\right) \cdot \mathbf{1},
$$

где $\mathcal{A}$ - квадратичный дифференциальный оператор, составленный из производных по временам, а функция $C(\alpha N)$ не зависит от времен. Вопрос о гипотетическом соответствии этих преобразований преобразованиям одевания иерархии КдФ остается открытым (его решение, в частности, позволило бы выразить соответствуюшие уравнения иерархии только в терминах моментов, как это сделано в случае MMK, что, в свою очередь, позволило бы вычислять базовые индексы пересечений (1.8) на ДПМ).

\section{2. ПРОСТРАНСТВА МОДУЛЕЙ И МАТРИЧНЫЕ МОДЕЛИ}

\section{1. Непрерывное пространство модулей $\mathcal{M}_{g, n}$.}

2.1.1. Обозначения. В дальнейшем $g$ и $n$ - целые числа, удовлетворяюшие условиям

$$
g \geqslant 0, \quad n>0, \quad 2-2 g-n<0
$$

Обозначим через $\mathcal{M}_{g, n}$ пространство модулей гладких полных комплексных кривых $C$ рода $g$ с $n$ различными отмеченными точками $x_{1}, \ldots, x_{n}$, замыкание которого $\overline{\mathcal{M}}_{g, n}-$ гладкая компактификация типа Делиня-Мамфорда (ее конкретный вид обсудим ниже). Обозначим через $\mathcal{L}_{i}, i=1, \ldots, n$, линейные расслоения над $\overline{\mathcal{M}}_{g, n}$. Слой расслоения $\mathcal{L}_{i}$ в точке $\left(C, x_{1}, \ldots, x_{n}\right)$ - это кокасательное пространство $T_{x_{i}}^{*} C$.

Пусть $d_{1}, \ldots, d_{n}$ - неотрицательные целые числа, удовлетворяющие условию

$$
\sum_{i=1}^{n} d_{i}=\operatorname{dim}_{\mathbb{C}} \overline{\mathcal{M}}_{g, n}=3 g-3+n .
$$

Тогда $\left\langle\tau_{d_{1}} \ldots \tau_{d_{n}}\right\rangle_{g}$ обозначает индекс пересечений, определяемый формулой (1.2), в которой $\omega_{i} \equiv c_{1}\left(\mathcal{L}_{i}\right)$ - первые классы Черна соответствуюших линейных расслоений над пространством модулей $\overline{\mathcal{M}}_{g, n}$.

Все матричные интегралы (если не указано обратное) предполагаются интегралами по эрмитовым матрицам со стандартной мерой интегрирования

$$
D X=\prod_{i<j}^{M} d \Re X_{i j} d \Im X_{i}
$$

для матриц размера $M \times M$.

Мы будем использовать известные результаты матричного интегрирования. В 1ММ можно проинтегрировать по угловым переменным (унитарным степеням свободы), после чего останется только интеграл по $M$ степеням свободы - собственным значениям 
матрицы $X$ :

$$
\begin{aligned}
\mathcal{Z}_{H}\left(\left\{\xi_{n}\right\}, M\right) & =\int_{M \times M} D X \exp \{-\operatorname{tr} V(X)\}= \\
& =(2 \pi)^{\frac{M^{2}-M}{2}} \prod_{i=1}^{M}(i !)^{-1} \int \prod_{i=1}^{M} d x_{i} \Delta(x)^{2} \exp \left\{-\sum_{i=1}^{M} V\left(x_{i}\right)\right\},
\end{aligned}
$$

где

$$
\Delta(x)=\prod_{i>j}^{M}\left(x_{i}-x_{j}\right)
$$

- определитель Вандермонда. Этот интеграл может быть представлен через ортогональные полиномы $\left\{P_{n}(x)\right\}_{n=0}^{\infty}$, связанные с потенциалом $V(x)=\sum_{n} \xi_{n} x^{n}$ :

$$
\int P_{n}(x) P_{m}(x) e^{-V(x)} d x=h_{n} \delta_{n, m}, \quad P_{n}(x)=x^{n}+a_{n-1}^{(n)} x^{n-1}+\cdots+a_{0}^{(n)} .
$$

Тогда с точностью до константы

$$
\mathcal{Z}_{H}=\prod_{i=1}^{M} h_{i}
$$

Другой используемый нами матричный интеграл - это интеграл Мехты-ИтциксонаЗубера по угловым переменным (унитарным матрицам) в модели с внешним (матричным) полем $\Lambda$ - эрмитовой матрицей с собственными значениями $\lambda_{i}, i=1, \ldots, M$ :

$$
\begin{aligned}
& \int_{M \times M} D X \exp \{-\operatorname{tr} U(X)+\operatorname{tr} \Lambda X\}= \\
& =(2 \pi)^{\frac{M^{2}-M}{2}} \int \prod_{i=1}^{M} d x_{i} \frac{\Delta(x)}{\Delta(\lambda)} \exp \left\{-\sum_{i=1}^{M}\left(U\left(x_{i}\right)-\lambda_{i} x_{i}\right)\right\}
\end{aligned}
$$

где $\Delta(x)$ и $\Delta(\lambda)$ - соответствуюшие определители Вандермонда.

2.1.2. Пространство модулей алгебраических кривых и его параметризация через дифференциалы Дженкинса-Штребеля. Квадратичный дифференциал Штребеля $\varphi$ на РП $C$ - это голоморфное сечение линейного расслоения $\left(T^{\star}\right)^{\otimes 2}$. В локальных координатах оно задает плоскую метрику на дополнении к дискретному множеству своих нулей и полюсов:

$$
d s^{2}=|\varphi(z)| \cdot|d z|^{2}, \quad \text { где } \quad \varphi=\varphi(z) d z^{2} .
$$

Все полюсы дифференциалов Штребеля - чисто двойные полюсы, размешенные в точках проколов. Кроме того, квадратичные вычеты $p_{i}^{2}$ в двойных полюсах должны быть строго положительными вешественными числами. Так как по теореме Римана для квадратичных дифференциалов \#нулей - \#полюсов $=4(g-1)$ (с учетом кратностей $)$, 


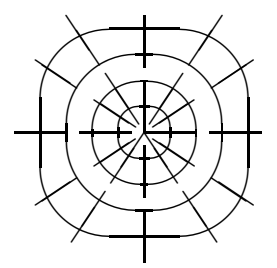

a

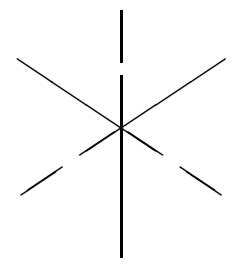

б

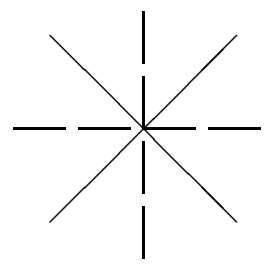

B

Рис. 1. Горизонтальные и вертикальные линии метрики Штребеля в полюсах и нулях.

то для точки обшего положения в $\mathcal{M}_{g, n}$ (РП с $n$ проколами) имеются ровно $4 g-4+2 n$ простых нулей.

Горизонтальная линия (геодезическая) квадратичного дифференциала - это кривая, вдоль которой дифференциал $\varphi(z) d z^{2}$ вешествен и положителен. Вертикальная линия (геодезическая), наоборот, - это линия, вдоль которой дифференциал $\varphi(z) d z^{2}$ веществен и отрицателен. Рассмотрим систему горизонтальных и вертикальных линий в окрестности двойного полюса, где $\varphi(z)=p_{i}^{2} /(2 \pi)^{2}\left(z-z_{i}\right)^{2}$ (рис. 1a). Легко видеть, что горизонтальные линии - это концентрические круги вокруг точки $z_{i}$, а вертикальные геодезические - лучи с вершиной в той же точке. Если взять нуль функции $\varphi(z)$ $k$-го порядка, то тогда имеются ровно $k+2$ горизонтальных и $k+2$ вертикальных лучей с вершиной в точке $z_{j}$ (рис. $1 б$ и 1в для $k=1$ и $k=2$, соответственно). Для дифференциала $\varphi$ общего вида горизонтальные линии не замкнуты, однако дифференииаль Дженкинса-Штребеля выделяются именно тем требованием, чтобы объединение незамкнутых горизонтальных линий имело бы нулевую меру. Это ровно те геодезические, которые соединяют нули дифференциала. Более того, они разбивают РП на односвязные компоненты (грани), в каждой из которых находится ровно одна выколотая точка. Наконец, длины всех горизонтальных траекторий, лежащих на одной грани, совпадают и равны $p_{i}$. Теорема Штребеля утверждает:

Теорема 1 [74]. Для любой связной $Р П C, n$ различных точек $x_{1}, \ldots, x_{n} \in C$ $(n>0$ nри $g>0$ и $n>2$ nри $g=0)$ и $n$ положстельных вещественных чисел $p_{1}, \ldots, p_{n}$ существует единственный квадратичный дифференциал (дифференциал Дженкинса-Штребеля) на $C \backslash\left\{x_{1}, \ldots, x_{n}\right\}$, чьи максимальные круговые области суть п проколотых дисков $D_{i}, x_{i} \in D_{i}$, имеющих периметры $p_{i}$.

Совокупность всех незамкнутых горизонтальных линий и есть ориентированный граф̆, отвечающий РП. Ориентация в данном случае означает, что ребра графа можно представить как ленты, две стороны которых принадлежат двум граням поверхности, разделенным данным ребром. В дальнейшем в технике матричных моделей индекс $i$ будет пробегать вдоль границы грани (см. рис. 2).

В метрике Штребеля каждая грань превращается в полубесконечный цилиндр с гранищей, состоящей из краев полос ленточного графа, т.е. граница $i$-й грани представляет собой многоугольник с периметром $p_{i}$.

Снабдим ориентированный граф дополнительной структурой: присвоим каждому ребру положительное вешественное число $l_{s} \in \mathbb{R}^{+}$, где $s-$ номер ребра. Эти числа $l_{s}-$ 


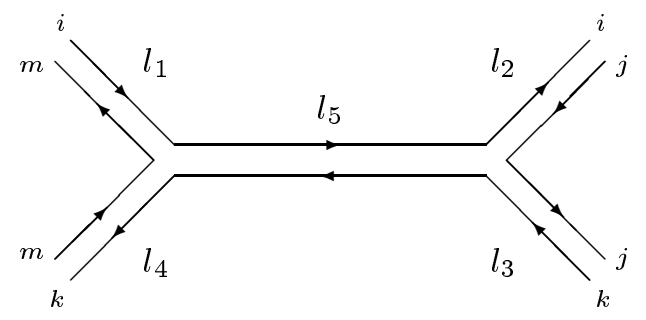

Рис. 2. Типичный подграф̆ в матричной модели с трехточечными вершинами.

длины ребер ленточного граф̆а $\Gamma_{g}$ рода $g$ - задают координаты на $(6 g-6+3 n)$-мерном линейном пространстве метризованных графов. Набор всех метризованных графов с точностью до симметрийных групп графа образует комбинаторное пространство модулей $\mathcal{M}_{g, n}^{\text {comb }}$. При этом периметры $p_{i}$ - это суммы длин ребер, инцидентных $i$-му циклу. Размерность $\mathcal{M}_{g, n}^{\text {comb }}$ равна сумме вешественной размерности пространства модулей $\mathcal{M}_{g, n}$ и $n$ дополнительных измерений - периметров граней. Структуры на самом компактифицированном пространстве модулей $\overline{\mathcal{M}}_{g, n}$ не должны зависеть от этих дополнительных параметров. Каждому дифференциалу Дженкинса-Штребеля сопоставляется некоторьй граф. Обратное утверждение также верно: произвольному графу отвечает некоторая РП с дифференциальной структурой Дженкинса-Штребеля и с квадратичными вычетами, равными квадратам периметров циклов графа. Верна следующая

Теорема 2 [56], [55], [75]. Пусть $\mathcal{M}_{g, n}^{\text {comb }}$ - множсество классов әквивалентности связных ориентированных метризованных графов с валентностью каждой вериины больше или равной 3 . Отображсние $\mathcal{M}_{g, n} \otimes \mathbb{R}_{+}^{n} \rightarrow \mathcal{M}_{g, n}^{\text {comb }}$, которое сопоставляет РП С и положительным числам $p_{1}, \ldots, p_{n}$ критический граф канонического дифференциала Дженкинса-Штребеля, является взаимно однозначным.

Таким образом, рассматривая совокупность всех графов с фиксированными $g$ и $n$ и всеми возможными (ненулевыми) длинами ребер, получаем стратификацию $\mathcal{M}_{g, n}^{\text {comb }}$ с размерностями страт, равными числу ребер соответствующего графа. Открытые страты отвечают комбинаторным типам трехвалентных графов и имеют размерность $6 g-$ $6+3 n$.

2.1.3. Геометрия расслоений на $\overline{\mathcal{M}}_{g, n}$ и матричный интеграл. Концевич [9] предложил способ нахождения индексов пересечений (или, что эквивалентно, интегралов от первых классов Черна) на пространствах модулей. Периметр $k$-й граничной компоненты

$$
p_{k}=\sum_{l_{\alpha} \in I_{k}} l_{\alpha}
$$

Тогда удобный выбор представителя замкнутой 2-формы $\omega_{k}$ (или первого класса Черна $\left.c_{1}\left(\mathcal{L}_{k}\right)\right)$ на этой компоненте имеет вид

$$
\omega_{k}=\sum_{1 \leqslant i<j \leqslant I_{k}} d\left(\frac{l_{i}}{p}\right) \wedge d\left(\frac{l_{j}}{p}\right)
$$


в предположении сушествования компактификаций пространств модулей, согласованных с клеточным разбиением.

Обозначим через $\pi: \mathcal{M}_{g, n}^{\mathrm{comb}} \rightarrow \mathbb{R}_{+}^{n}$ проекцию на пространство периметров. Теперь индексы пересечений задаются формулой

$$
\left\langle\tau_{d_{1}} \ldots \tau_{d_{n}}\right\rangle=\int_{\pi^{-1}\left(p_{*}\right)} \prod_{i=1}^{n} \omega_{i}^{d_{i}}
$$

где $p_{*}=\left(p_{1}, \ldots, p_{n}\right)-$ произвольная последовательность положительных вешественных чисел, $\pi^{-1}\left(p_{*}\right)$ - слой $\overline{\mathcal{M}}_{g, n}$ в $\mathcal{M}_{g, n}^{\text {comb }}$.

Обозначим через $\Omega$ 2-форму на открытых стратах пространства $\mathcal{M}_{g, n}^{\mathrm{comb}}$ :

$$
\Omega=\sum_{k=1}^{n} p_{k}^{2} \omega_{k}
$$

ограничения которой на слои проекции $\pi$ имеют постоянные коэффициенты в координатах $l_{e}$. Пусть $d=3 g-3+n-$ комплексная размерность пространства $\mathcal{M}_{g, n}$. Тогда объем слоя проекции $\pi$ по отношению к форме $\Omega$ равен

$$
\begin{aligned}
\operatorname{vol}\left(\pi^{-1}\left(p_{1}, \ldots, p_{n}\right)\right) & =\int_{\pi^{-1}\left(p_{*}\right)} \frac{\Omega^{d}}{d !}=\frac{1}{d !} \int_{\pi^{-1}\left(p_{*}\right)}\left(p_{1}^{2} c_{1}\left(\mathcal{L}_{1}\right)+\cdots+p_{n}^{2} c_{1}\left(\mathcal{L}_{n}\right)\right)^{d}= \\
& =\sum_{\sum d_{i}=d} \prod_{i=1}^{n} \frac{p_{i}^{2 d_{i}}}{d_{i} !}\left\langle\tau_{d_{1}} \ldots \tau_{d_{n}}\right\rangle_{g}
\end{aligned}
$$

Сделаем важное замечание. Согласно теореме Конщевича все эти интегрирования непрерывно продолжаются на замыканиепространства модулей $\overline{\mathcal{M}}_{g, n}$ по процедуре Делиня и Мамфорда [76], [77] (т.е. рассматривается стабильный когомологический класс кривых). Замыкание пространства модулей необходимо, так как пересечения вообше корректно определены лишь на компактных пространствах. Однако это обстоятельство не сказывается на результатах до тех пор, пока интегрирование ведется по непрерывному пространству модулей, где ненулевые вклады дают только интегрирования по клеткам старшей размерности, поскольку все дополнительные симплексы, добавляемые для замыкания $\mathcal{M}_{g, n}$, имеют низшие размерности. (Тем не менее эти вклады становятся сушественными в случае дискретных пространств модулей.)

Чтобы понять природу возникновения матричной модели, сделаем преобразование Лапласа по переменным $p_{i}$ от объемов слоев проекции $\pi$ для величин в правой части формулы (2.8):

$$
\int_{0}^{\infty} d p_{i} \exp \left(-p_{i} \lambda_{i}\right) p_{i}^{2 d_{i}}=\left(2 d_{i}\right) ! \lambda_{i}^{-2 d_{i}-1} .
$$

В левой части получим

$$
\int_{0}^{\infty} \cdots \int_{0}^{\infty} d p_{1} \wedge \cdots \wedge d p_{n} \exp \left(-\sum p_{i} \lambda_{i}\right) \int_{\overline{\mathcal{M}}_{g, n}} e^{\Omega}
$$


и ввиду сокрашения всех множителей $p_{i}^{2}$ из знаменателей формы $\Omega$ имеем

$$
e^{\Omega} d p_{1} \wedge \cdots \wedge d p_{n}=\rho \prod_{e \in X_{1}} d l_{e}
$$

Обозначим через $\# X_{q}$ полное число $q$-мерных клеток симплициального комплекса (\# $X_{1}$ - число ребер, \# $X_{0}$ - число вершин, и т.д.). Функция $\rho$-положительная функция, определенная на открытых клетках:

$$
\rho=\left(\prod_{i=1}^{n}\left|d p_{i}\right| \times \frac{\Omega^{d}}{d !}\right): \prod_{e \in X_{1}}\left|d l_{e}\right|
$$

Константа $\rho$ зависит лишь от эйлеровой характеристики графа $\Gamma$,

$$
\rho=2^{d+\# X_{1}-\# X_{0}} .
$$

Интеграл

$$
I_{g}\left(\lambda_{*}\right):=\int_{\mathcal{M}_{g, n}^{\text {comb }}} \exp \left(-\sum_{i} \lambda_{i} p_{i}\right) \prod_{e \in X_{1}}\left|d l_{e}\right|
$$

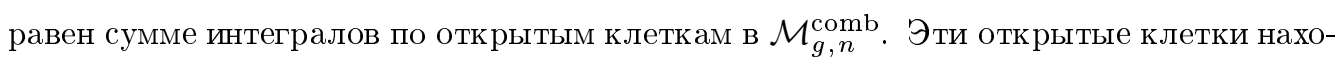
дятся во взаимно однозначном соответствии с полным набором трехвалентных графов, даюших вклад в данньй порядок разложения по $g$ и $n$. Необходимо также учесть внутренние автоморфизмы графа (их число показывает, сколько копий пространства модулей находится внутри клетки). Наконец, сумма $\sum_{i} \lambda_{i} p_{i}$ выражается через $l_{e}$ :

$$
\sum_{i=1}^{n} \lambda_{i} p_{i}=\sum_{e \in X_{1}} l_{e}\left(\lambda_{e}^{(1)}+\lambda_{e}^{(2)}\right)
$$

для каждого графа. Здесь $\lambda_{e}^{(1)}$ и $\lambda_{e}^{(2)}$-переменные двух циклов, иншидентных $e$-му ребpy (см., например, рис. 2). Делая теперь преобразование Лапласа, получим соотношение [9]

$$
\sum_{d_{1}, \ldots, d_{n}=0}^{\infty}\left\langle\tau_{d_{1}} \ldots \tau_{d_{n}}\right\rangle_{g} \prod_{i=1}^{n}\left(2 d_{i}-1\right) ! ! \lambda_{i}^{-\left(2 d_{i}+1\right)}=\sum_{\Gamma} \frac{2^{-\# X_{0}}}{\# \operatorname{Aut}(\Gamma)} \prod_{\{i j\}} \frac{2}{\lambda_{i}+\lambda_{j}}
$$

где в правой части суммирование ведется по всем ориентированным связным трехвалентным ленточным графам $\Gamma$ с $n$ отмеченными граничными компонентами, произведение берется по всем ребрам графа, \# $X_{0}$ - число вершин графа $\Gamma$, \# Aut - объем дискретной группы симметрий графа $Г$.

Ключевое наблюдение Концевича состояло в том, что величина в правой части уравнения (2.16) - это свободная энергия матричной модели (ММК). 
Теорема 3 (Концевич [9]). Рассматривая матричные интеграль по эрмитовым $(N \times N)$-матрицам как асимптотические разложсения по временам

$$
t_{n}=\frac{1}{2 n+1} \operatorname{tr} \frac{1}{\lambda^{2 n+1}},
$$

$n=0,1,2, \ldots, \quad(-1) ! ! \equiv 1$, получим

$$
\begin{gathered}
\sum_{\substack { g=0 \\
\begin{subarray}{c}{n=1, g>0 \\
n=3, g=0{ g = 0 \\
\begin{subarray} { c } { n = 1 , g > 0 \\
n = 3 , g = 0 } }\end{subarray}}^{\infty} \frac{1}{(\alpha N)^{2 g-2+n}} \sum_{\Sigma d_{i}=3 g-3+n}\left\langle\tau_{d_{1}} \ldots \tau_{d_{n}}\right\rangle_{g} \frac{1}{n !} \prod_{i=1}^{n}\left(2 d_{i}+1\right) ! ! t_{d_{i}}= \\
=\ln \frac{\int D X \exp \left\{-\alpha N \operatorname{tr}\left(\frac{X^{2} \Lambda}{2}+\frac{X^{3}}{6}\right)\right\}}{\int D X \exp \left\{-\alpha N \operatorname{tr} \frac{X^{2} \Lambda}{2}\right\}}= \\
=\mathcal{F}_{\mathrm{K}}\left(t_{0}, t_{1}, \ldots\right) \equiv \ln \mathcal{Z}_{\mathrm{K}}\left(\left\{t_{n}\right\}, \alpha N\right), \\
\Lambda=\operatorname{diag}\left\{\lambda_{1}, \ldots, \lambda_{N}\right\} .
\end{gathered}
$$

Правила Фейнмана для MМК имеют следуюший вид: как и в обычных матричных моделях, пропагаторы ленточных графов имеют две стороны, каждая из которых несет соответствующий индекс (рис. 2). ММК отличается от 1ММ дополнительными переменными $\lambda_{i}$, ассоциированными с петлями индекса $i$ в диаграмме, причем пропагатор равен $2 /\left(\lambda_{i}+\lambda_{j}\right)$, где $\lambda_{i}$ и $\lambda_{j}$ - переменные двух циклов (возможно, одного и того же цикла), инцидентных двум сторонам ленточного ребра. Все вершины трехвалентны, что отвечает клеткам старшей размерности в клеточном разложении пространства модулей.

Рассмотрим наиболее простой пример графа рода ноль с тремя граничными компонентами, несушими переменные $\lambda_{1}, \lambda_{2}$ и $\lambda_{3}$. В этом порядке дают вклад два типа диаграмм (рис.3). После симметризации по переменным $\lambda_{1}, \lambda_{2}$ и $\lambda_{3}$ вклад в свободную энергию от суммы всех диаграмм равен

$$
\begin{aligned}
& \frac{1}{6\left(\lambda_{1}+\lambda_{2}\right)\left(\lambda_{1}+\lambda_{3}\right)\left(\lambda_{2}+\lambda_{3}\right)}+ \\
& \quad+\frac{1}{3}\left\{\frac{1}{4 \lambda_{1}\left(\lambda_{2}+\lambda_{1}\right)\left(\lambda_{3}+\lambda_{1}\right)}+(1 \rightarrow 2,2 \rightarrow 3,3 \rightarrow 1)+(1 \rightarrow 3,3 \rightarrow 2,2 \rightarrow 1)\right\}= \\
& =\frac{2 \lambda_{1} \lambda_{2} \lambda_{3}+\lambda_{2} \lambda_{3}\left(\lambda_{2}+\lambda_{3}\right)+\lambda_{1} \lambda_{3}\left(\lambda_{1}+\lambda_{3}\right)+\lambda_{1} \lambda_{2}\left(\lambda_{1}+\lambda_{2}\right)}{12 \lambda_{1} \lambda_{2} \lambda_{3}\left(\lambda_{1}+\lambda_{2}\right)\left(\lambda_{1}+\lambda_{3}\right)\left(\lambda_{2}+\lambda_{3}\right)}=\frac{1}{12 \lambda_{1} \lambda_{2} \lambda_{3}} . \quad(2.18)
\end{aligned}
$$

На этом примере видно, как члены $\left(\lambda_{i}+\lambda_{j}\right)$ сокрашаются в знаменателе.

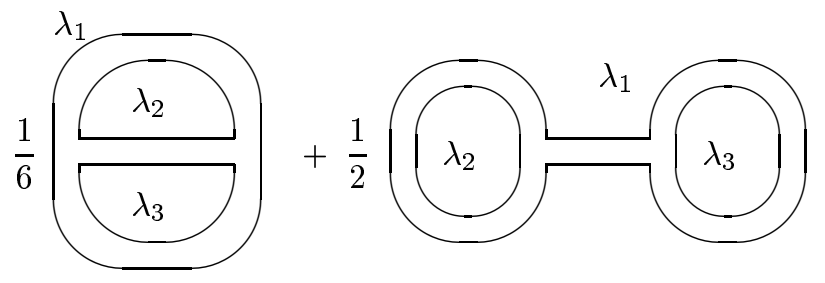

Рис. 3. Диаграммы MMK, дающие вклад в порядке $g=0, n=3$. 
2.2. Дискретизованное пространство модулей $\overline{\mathcal{M}}_{g, n}^{\text {disc }}$. Опишем дискретизацию пространств модулей. Хотя полное математическое обоснование этой процедуры до сих пор отсутствует, указанием на ее справедливость может служить тот факт, что эти пространства описываются с помошью другой точно решаемой матричной модели, а именно ММКП (1.9).

2.2.1. Матричная модель Конщевича-Пеннера. Рассмотрим матричную модель (1.9) [54], [62]. В отличие от ММК ее потенциал включает все члены $X^{n}$, так как он прямо описьвает разбиение пространства модулей на клетки симплициального комплекса и сумма идет по всем симплексам возможных размерностей. (На языке MМК ме́ньшие размерности отвечают редушированию (стягиванию в точку) все большего числа ребер ленточного графа.) Присутствие логарифмического потенциала делает эту модель похожей на матричную модель Пеннера (1.6) [56].

Найдем правила Фейнмана для модели (1.9). Прежде всего, в ней, как и в модели Пеннера, имеются вершины всех порядков по $X$. Из-за врашательной симметрии множитель $1 / n$, стояший при $X^{n}$, сокрашается; при этом остается только симметрийньй множитель $1 / \#$ Aut $\Gamma$. Кроме того, при каждой вершине стоит множитель $\alpha / 2$. Как и в MMK, с каждым циклом ассоциируется переменная $\lambda_{i}$. Однако форма пропагатора изменяется: вместо $2 /\left(\lambda_{i}+\lambda_{j}\right)$ получим $2\left(e^{\lambda_{i}+\lambda_{j}}-1\right) / \alpha$.

Рассмотрим тот же случай $(g=0, n=3)$, что и в ММК. Помимо приведенных выше возникает одна новая диаграмма, отвечаюшая вершине $X^{4}$ (рис. 4).

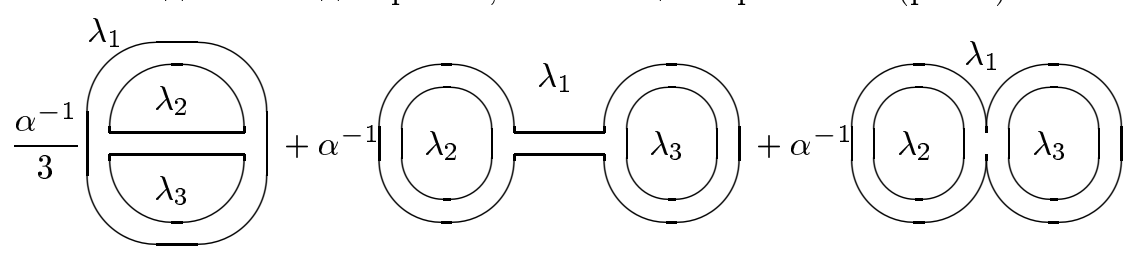

Рис. 4. Вклад порядка $g=0, n=3$ в модель (1.9).

После симметризации по $\lambda_{1}, \lambda_{2}$ и $\lambda_{3}$ получим ответ

$$
F_{0,3}=N^{2} \alpha^{-1} \frac{e^{\lambda_{1}+\lambda_{2}}+e^{\lambda_{1}+\lambda_{3}}+e^{\lambda_{2}+\lambda_{3}}+1}{3\left(e^{2 \lambda_{1}}-1\right)\left(e^{2 \lambda_{2}}-1\right)\left(e^{2 \lambda_{3}}-1\right)},
$$

в котором, как и в МMК, происходит сокращение перекрестных членов в знаменателе. "Техническая" причина, по которой модель (1.9) содержит зависимость только от $\operatorname{tr} \Lambda^{k}$ $(k \leqslant 0)$, состоит в том, что эта модель, как и ММК, относится к классу ОМК $(1.5)$ [42]-[44]. Это означает, что после некоторого (достаточно простого) преобразования модель (1.9) сведется к модели с потенциалом $\eta X+V(X)$, которая зависит только от времен Мивы.

2.2.2. Дискретизованные пространства модулей. Дискретизация пространств модулей $\overline{\mathcal{M}}_{g, n}$ и $\mathcal{M}_{g, n}^{\text {comb } п р е д п о л а г а е т ~ р а з р е ш е н н ы м и ~ с л е д у ю ш и е ~ з н а ч е н и я ~ п а р а м е т р о в ~} l_{i}$ :

$$
l_{i} \in \mathbb{Z}_{+} \cup\{0\}, \quad p_{i} \in \mathbb{Z}_{+}, \quad \sum_{i=1}^{n} p_{i} \in 2 \mathbb{Z}_{+} .
$$


Таким образом, все числа $l_{i}$ и $p_{i}$ будут целыми, но некоторые из $l_{i}$ могут обрашаться в нуль, в то время как все периметры остаются строго положительными. Сумма всех периметров четн $a^{3)}$, поскольку каждое ребро считается дважды при вычислении этой суммы. Такое (комбинаторное) пространство называется $\overline{\mathcal{M}}_{g, n}^{\text {disc }}$. Следует отметить, что теперь в рассмотрение явно включены те точки исходного пространства $\overline{\mathcal{M}}_{g, n}$, которые являются точками редукций (сингулярные кривые, лежашие на гранище (замыкании) $\partial \mathcal{M}_{g, n}$ ) и кривизны (орбифолдные точки, стабильные при действии некоторой конечной неединичной подгруппы (стабилизируюшей подгруппы) группы симметрий пространства Тейхмюллера). Через $\mathcal{M}_{g, n}^{\text {disc }}$ обозначим такое подмножество $\overline{\mathcal{M}}_{g, n}^{\text {disc }}$, из которого исключены все точки редукций. Внешняя производная $d$ заменяется на конечно-разностный оператор $\tilde{d}$, а интегрирование - на поточечное суммирование [52].

На $k$-й граничной компоненте 2-форма $\widetilde{\omega}$ имеет вид

$$
\widetilde{\omega}_{k}=\sum_{1 \leqslant i<j \leqslant I_{k}} \frac{\tilde{d} l_{i}}{p_{k}} \wedge \frac{\tilde{d} l_{j}}{p_{k}} .
$$

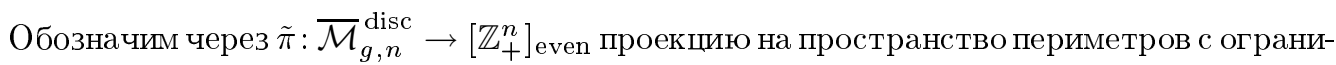
чением $\sum_{i} p_{i} \in 2 \mathbb{Z}_{+}$. Тогда индексы пересечений определятся как

$$
\left\langle\left\langle\tau_{d_{1}} \ldots \tau_{d_{n}}\right\rangle\right\rangle_{g}=\int_{\tilde{\pi}^{-1}\left(p_{*}\right)} \prod_{i=1}^{n} \widetilde{\omega}_{i}^{d_{i}}
$$

где $p_{*}=\left(p_{1}, \ldots, p_{n}\right)-$ произвольная последовательность положительных целых чисел, сумма $\sum_{i=1}^{n} p_{i}$ обязательно четна, а $\tilde{\pi}^{-1}\left(p_{*}\right)$ - аналог сечения пространства модулей $\overline{\mathcal{M}}_{g, n}$ в $\overline{\mathcal{M}}_{g, n}^{\text {disc }}$. Заметим, однако, что объем слоя $\tilde{\pi}^{-1}\left(p_{*}\right)$ не обязательно однороден по $p_{i}$ ввиду возможной ненулевой кривизны накрьвающего многообразия. Таким образом, индексы (2.22) могут быть отличны от нуля и при $\sum_{i} d_{i} \leqslant d \equiv 3 g-3+n$.

Важно отметить, что каждое сечение $\tilde{\pi}^{-1}\left(p_{*}\right)$ содержит конечное множество точек, тем самым эти сечения не изоморфны друг другу. Однако все они - аналоги одного и того же исходного пространства модулей $\overline{\mathcal{M}}_{g, n}$ с различными вспомогательными периметрами. Поэтому естественно называть эти пространства дискретизованнылми пространствами модулей. Соотношение (2.22) не зависит от числа точек исходного пространства $\overline{\mathcal{M}}_{g, n}$, входящих в сечение $\tilde{\pi}^{-1}\left(p_{*}\right)$ (это может быть даже единственная точка редукции, что имеет место, например, для $\left.\overline{\mathcal{M}}_{1,1}\right)$. Значения этих индексов пересечений рациональные числа, что отражает орбифолдную природу исходного пространства модулей $\overline{\mathcal{M}}_{g, n}$. Так как симметрийные свойства графов не изменились, то значения старших когомологических классов, для которых $\sum_{i} d_{i}=d$ в $(2.22)$, не должны зависеть ни от значений периметров, ни от того, какое рассматривается пространство модулей непрерывное или дискретное.

\footnotetext{
3) Это вполне тривиальное наблюдение оказывается довольно важным. Так, из него следует удвоение числа времен данной модели.
} 
2.2.3. Матричный интеграл для дискретизованного пространства модулей. Пусть

$$
\widetilde{\Omega}=\sum_{i=1}^{n} p_{i}^{2} \widetilde{\omega}_{i}
$$

- 2-форма на $\overline{\mathcal{M}}_{g, n}^{\text {disc }}$, ограничения которой на сечения $\tilde{\pi}$ имеют постоянные коэффициенты в координатах $l_{e}$. Объем сечения $\tilde{\pi}$, взятого по $\widetilde{\Omega}$, представим в виде

$$
\begin{aligned}
\operatorname{vol}\left(\tilde{\pi}^{-1}\left(p_{1}, \ldots, p_{n}\right)\right) & =\int_{\tilde{\pi}^{-1}\left(p_{*}\right)} \frac{\widetilde{\Omega}^{d}}{d !}=\frac{1}{d !} \int_{\tilde{\pi}^{-1}\left(p_{*}\right)}\left(p_{1}^{2} \widetilde{\omega}_{1}+\cdots+p_{n}^{2} \widetilde{\omega}_{n}\right)^{d}= \\
& =\sum_{\sum d_{i} \leqslant d} \prod_{i=1}^{n} \frac{p_{i}^{2 d_{i}}}{d_{i} !}\left\langle\left\langle\tau_{d_{1}} \ldots \tau_{d_{n}}\right\rangle\right\rangle_{g}, \quad d=3 g-3+n
\end{aligned}
$$

Произведем преобразования Лапласа в обеих частях (2.24), заменяя непрерывное преобразование Лапласа дискретным и явно учитывая тот факт, что сумма всех $p_{i}$ четна. В правой части получим

$$
\begin{aligned}
& \sum_{\substack{p_{i} \in \mathbb{Z}_{+} \\
\sum p_{i} \in 2 \mathbb{Z}_{+}}} \exp \left\{-\sum_{i} p_{i} \lambda_{i}\right\} p_{1}^{2 d_{1}} \ldots p_{n}^{2 d_{n}}= \\
& \quad=\prod_{i=1}^{n}\left(\frac{\partial}{\partial \lambda_{i}}\right)^{2 d_{i}} \cdot \frac{1}{2}\left\{\prod_{i=1}^{n} \frac{1}{e^{\lambda_{i}}-1}+(-1)^{n} \prod_{i=1}^{n} \frac{1}{e^{\lambda_{i}}+1}\right\} .
\end{aligned}
$$

В то же время в левой части (2.24) снова сделаем подстановку

$$
\left.e^{\tilde{\Omega}} \tilde{d} p_{1} \wedge \cdots \wedge \tilde{d} p_{n}\right|_{\sum p_{i} \in 2 \mathbb{Z}_{+}}=\tilde{\rho} \prod_{e \in X_{1}} \tilde{d} l_{e}
$$

где константа $\tilde{\rho}$ - отношение мер, аналогичное (2.13). С учетом четности суммы всех $p_{i}$ получим конечную перенормировку $\rho$ в случае ДПМ:

$$
\tilde{\rho}=\frac{\rho}{2},
$$

где $\rho$ дается формулой (2.13).

Теперь дадим матрично-модельное описание этих "новых" индексов пересечений. Рассмотрим более подробно соответствие между графами и различными точками пространств $\tilde{\pi}^{-1}\left(p_{*}\right)$. Отметим, что точки общего положения со всеми $l_{i}$, бо́льшими нуля, отвечают графам с чисто трехвалентными вершинами. Кроме того, имеются такие точки $\tilde{\pi}^{-1}\left(p_{*}\right)$, где некоторые из $l_{i}$ обрашаются в нуль, но сами эти точки еше не соответствуют редукциям. Пример тора с одной отмеченной точкой показан на рис. 5, где граф̆ на рис. 5а отвечает точке обшего положения $\left(l_{i}>0, i=1,2,3\right)$, а граф на рис. $5 б$ отвечает случаю, когда одно (и только одно) из чисел $l_{i}$ обрашается в нуль. (В параметризации Тейхмюллера рис. $5 б$ соответствует тору с чисто мнимым модулярным параметром $\tau$.) 


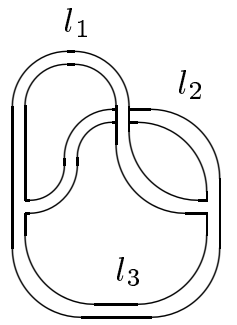

a

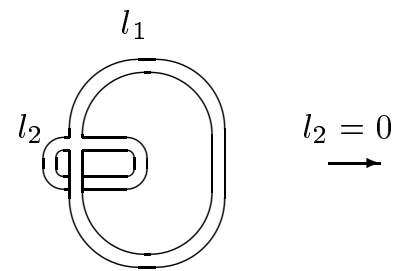

б

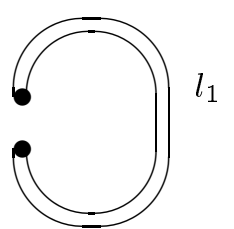

B

Рис. 5. Диаграммы, отвечающие различным клеткам симплициального комплекса пространства $\overline{\mathcal{M}}_{1,1}$.

Однако в каждой области $\tilde{\pi}^{-1}\left(p_{*}\right)$ (кроме случая $\left.\mathcal{M}_{0,3}\right)$ имеются "подлинные" точки редукции (см., например, рис. 5в, где две переменные $l_{i}$ обрашаются в нуль). Таким кривым невозможно сопоставить никакой граф.

Поскольку не существует графов, прямо описьвающих редуцированные кривые, то необходимо каким-либо образом “исключить" такие точки редукции из $\tilde{\pi}^{-1}\left(p_{*}\right)$, при этом соотношение (2.22) должно модифицироваться. Пусть $\mathcal{M}_{g, n}^{\text {disc }}$ - такое подмножество $\overline{\mathcal{M}}_{g, n}^{\text {disc }}$, из которого исключены все точки редукции. "Выделение" интегрирования по открытому множеству $\mathcal{M}_{g, n}$ из полного интегрирования по $\overline{\mathcal{M}}_{g, n}$ происходит с использованием процедуры стратификации по Делиню и Мамфорду [76], [77]. Идея состоит в том, чтобы представить открытое пространство модулей $\mathcal{M}_{g, n}$ в виде некоторой комбинации $\overline{\mathcal{M}}_{g, n}$ и $\overline{\mathcal{M}}_{g_{j}, n_{j}}$ низших размерностей (подробнее см. [21], [78]).

Общая процедура, выражаюшая $\mathcal{M}_{g, n}$ через замкнутые пространства модулей, состоит в следуюшем: надо построить альтернированную сумму

$$
\mathcal{M}_{g, n}=\sum_{r_{q}=0}^{3 g-3+n}(-1)^{r_{q}} c_{g, n, r_{q}} \bigotimes_{j=1}^{q} \overline{\mathcal{M}}_{g_{j}, n_{j}+k_{j}},
$$

где суммирование ведется по всем $q$-компонентным редукциям, $r_{q}$ - степень редукции, $c_{g, n, r_{q}}$ - симметрийные коэффициенты, $k_{j}$ - число дополнительных проколотых точек, возникаюших благодаря редукциям. Размерность $\overline{\mathcal{M}}_{g_{j}, n_{j}+k_{j}}$ равна $d_{j}=$ $3 g_{j}-3+n_{j}+k_{j}$

$$
\sum_{j=1}^{q} n_{j}=n, \quad \sum_{j=1}^{q} d_{j}=d-r_{q}
$$

Тем самым получим

$$
\begin{aligned}
\int_{\mathcal{M}_{g, n}^{\text {disc }}} e^{\tilde{\Omega}} \exp \left\{\sum_{i} \lambda_{i} p_{i}\right\} \tilde{d} p_{1} \wedge \cdots \wedge \tilde{d} p_{n}= \\
=\frac{1}{d !} \int_{\overline{\mathcal{M}}_{g, n}^{\text {disc }}}\left(\sum_{i=1}^{n} p_{i}^{2} \widetilde{\omega}_{i}\right)^{d} \exp \left\{\sum_{i} \lambda_{i} p_{i}\right\} \tilde{d} p_{1} \wedge \cdots \wedge \tilde{d} p_{n}+ \\
\quad+\sum_{r_{q}=1}^{3 g-3+n}(-1)^{r_{q}} \bigotimes_{j=1}^{q} \int_{\overline{\mathcal{M}}_{g_{j}, n_{j}+k_{j}}}\left(\sum_{a=1}^{n_{j}} p_{a}^{2} \widetilde{\omega}_{a}\right)^{d_{j}} \exp \left\{\sum_{i} \lambda_{i} p_{i}\right\} \tilde{d} p_{1} \wedge \cdots \wedge \tilde{d} p_{n_{j}} .
\end{aligned}
$$


Теперь с использованием (2.26) найдем описание левой части формулы (2.30) через матричную модель. Как и в непрерывном случае, получим

$$
\text { Левая часть }(2.30)=\int_{\mathcal{M}_{g, n}^{\text {disc }}} \exp \left\{-\sum_{e \in X_{1}} l_{e}\left(\lambda_{e}^{(1)}+\lambda_{e}^{(2)}\right)\right\} \tilde{\rho} \prod_{e \in X_{1}}\left|\tilde{d} l_{e}\right|,
$$

где $\tilde{\rho}=2^{d+\# X_{1}-\# X_{0}-1}$. Выражение $(2.31)$ представимо в виде суммы по всем возможным “ленточным графам" Г с вершинами всех возможных для данного рода $g$ и числа граней $n$ валентностей. Опять надо учесть объем группы автоморфизмов графа.Наконец, надо "проинтегрировать" по каждому $l_{e}$, т.е. вычислить сумму по всем положительным целым значениям $l_{e}$ (так как все нулевые значения $l_{e}$ учитываются при суммировании по всем графам). Получим

$$
\text { Левая часть }(2.30)=2^{d-1} \sum_{\substack{\text { все } \\ \text { графы } \Gamma}} \frac{1}{\# \operatorname{Aut}(\Gamma)} 2^{-\# X_{0}} \prod_{e \in X_{1}} \frac{2}{e^{\lambda_{e}^{(1)}+\lambda_{e}^{(2)}}-1} \text {. }
$$

Это не что иное, как член из разложения по родам матричной модели (1.9) с

$$
\tilde{\Lambda}=\operatorname{diag}\left\{e^{\lambda_{1}}, \ldots, e^{\lambda_{N}}\right\}
$$

Тогда $\ln \mathcal{Z}[\Lambda]$ допускает следуюшее разложение по родам:

$$
\ln \mathcal{Z}[\Lambda]=\sum_{g=0}^{\infty} \sum_{n=1}^{\infty}(N \alpha)^{2-2 g} \alpha^{-n} w_{g}\left(\lambda_{1}, \ldots, \lambda_{n}\right) .
$$

Используем соотношение (2.25), чтобы выразить правую часть (2.30) через индексы пересечений:

$$
\begin{aligned}
& w_{g}\left(\lambda_{1}, \ldots, \lambda_{n}\right)=\frac{1}{2^{d-1}} \sum_{\substack{\text { редукции } \\
q \text {-компонентные }}}(-1)^{r_{q}} \times
\end{aligned}
$$

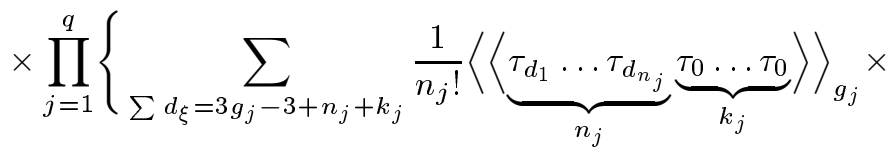

$$
\begin{aligned}
& \left.\times \operatorname{tr}\left[\prod_{k=1}^{n_{j}}\left(\frac{\partial}{\partial \lambda_{k}}\right)^{2 d_{k}}\right] \frac{1}{\left(d_{k}\right) !} \cdot \frac{1}{2}\left(\prod_{k=1}^{n_{j}} \frac{1}{e^{\lambda_{k}}-1}+(-1)^{n_{j}} \prod_{k=1}^{n_{j}} \frac{1}{e^{\lambda_{k}}+1}\right)\right\} .
\end{aligned}
$$

Тем самым получено следуюшее утверждение.

ТЕОРемА 4. В компактификационной схеме, совместной с параметризацией ШІтребеля пространства модулей $\mathcal{M}_{g, n}$, асимптотическое разложение по временам

$$
T_{k}^{ \pm}=\frac{1}{(k+1) !} \frac{1}{N} \sum_{i=1}^{N} \operatorname{tr} \frac{\partial^{k}}{\partial \lambda_{i}^{k}} \frac{1}{e_{i}^{\lambda} \pm 1}
$$


имеет вид

$$
\begin{aligned}
& \sum_{g=0}^{\infty} \sum_{\substack{n=1, g>0 \\
n=3, g=0}}^{\infty} \frac{1}{(\alpha N)^{2 g-2+n}} \sum_{\substack{\text { редукции } \\
q \text {-компонентные }}} c_{g, n, r_{q}}\left(-\frac{1}{2}\right)^{\left|r_{q}\right|} \times \\
& \times 2^{1-q} \prod_{j=1}^{q}\left\{\sum_{\sum d_{\xi}=3 g_{j}-3+n_{j}+k_{j}} \frac{1}{n_{j} !}\left\langle\langle\underbrace{\tau_{d_{1} \ldots \tau_{d_{n_{j}}}}}_{n_{j}} \underbrace{\tau_{0} \ldots \tau_{0}}_{k_{j}}\rangle\right\rangle_{g_{j}} \times\right. \\
& \left.\times\left(\prod_{k=1}^{n_{j}}\left(2 d_{j}+1\right) ! ! T_{2 d_{j}}^{-}+(-1)^{n_{j}} \prod_{k=1}^{n_{j}}\left(2 d_{j}+1\right) ! ! T_{2 d_{j}}^{+}\right)\right\}= \\
& =\ln \frac{\int D X \exp \left\{-\alpha N \operatorname{tr}\left(\frac{1}{4} \tilde{\Lambda} X \tilde{\Lambda} X+\frac{1}{2} \ln (1-X)+\frac{1}{2} X\right)\right\}}{\int D X \exp \left\{-\alpha N \operatorname{tr}\left(\frac{1}{4} \tilde{\Lambda} X \tilde{\Lambda} X-\frac{1}{4} X^{2}\right)\right\}}= \\
& =\mathcal{F}_{\mathrm{KP}}\left(\left\{T_{2 n}^{ \pm}\right\}\right) \equiv \mathcal{Z}_{\mathrm{KP}}\left(e^{\lambda}\right),
\end{aligned}
$$

$\tilde{\Lambda}$ определено в (2.33). Здесь суммирование ведется по всем редукииям, $c_{g, n, r_{q}}{ }_{-}$ положстельнье рациональные числа (коэффициенты редукции), $\left|r_{q}\right|$ - неотрицательные целье числа (степени редукции), $0 \leqslant\left|r_{q}\right| \leqslant 3 g-3+n$, и, наконец, $q$ - число компонент сингулярной кривой, $q$ изменяется от 1 до $2 g-2+n$. Вставки $k_{j}$ дополнительных $\tau_{0}$ в корреляционную функиию обусловлены этими редукииями.

Первый член в $(2.37)$ с $\left|r_{q}\right|=0, c_{g, n, r_{q}}=1$ и $q=1$ отвечает “старшему" нередуцированному модулярному пространству $\overline{\mathcal{M}}_{g, n}^{\text {disc }}$, из которого вычитаются интегралы по редукциям первого порядка. Эти интегралы, в свою очередь, являются замкнутыми пространствами модулей более низкой полной размерности, из них вычитаются интегралы, полученные в точках (само)пересечений этих замкнутых подпространств, и т.д.

2.2.4. Пространство модулей $\overline{\mathcal{M}}_{1,1}$. Рассмотрим пример модулярного пространства $\mathcal{M}_{1,1}$ - тора с одной выколотой точкой-в картине Концевича-Штребеля. В этом случае имеются три типа клеток симплициального комплекса, соответствующих диаграммам на рис. 5а-в. Симплициальный комплекс $\mathcal{T}_{1,1}$ изображен на рис. 6 . Отождествим ребра параллелограмма, получив таким образом сферу $\mathbb{S}^{2}$ с тремя особыми точками, т.е. орбифолд, составленный из двух открытых треугольников ${ }^{4)}$ (граф̆ на рис. 5а), трех ребер, разделяющих эти треугольники (граф на рис. 5б), и трех точек редукции - вершин треугольников (рис. 5в). (Единственная редукция тора с одной выколотой точкой - это сфера с тремя выколотыми точками, которой отвечает модулярное пространство $\mathcal{M}_{0,3}$, состоящее из всего лишш одной точки.) При этом каждая точка редукции берется с множителем $1 / 3$ (в соответствии с дефицитом угла). Центры треугольников, отмеченные на рис. 6 маленькими кружками, отвечают двум копиям тройной точки $\left(z=e^{i \pi / 3}\right.$ в картине Тейхмюллера), а центры ребер (маленькие окружности) - трем копиям квадратичной точки $\left(z=e^{i \pi / 2}\right.$ в картине Тейхмюллера). В орбифолде $\overline{\mathcal{M}}_{1,1}=\mathbb{S}^{2} / G_{1,1}$, где

\footnotetext{
4) Благодаря двум возможным выборам ориентации имеются две конгруэнтные клетки старшей размерности.
} 


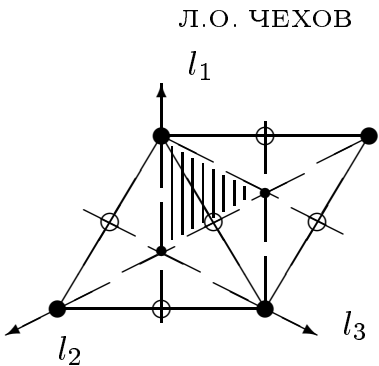

Рис. 6. Симплициальный комплекс для $\overline{\mathcal{M}}_{1,1}$ в картине Концевича-Штребеля.

$G_{1,1}$ - группа симметрий шестого порядка, содержится шесть копий исходной модулярной фигуры, одна из которых заштрихована на рис. 6.

В картине Концевича-Штребеля орбифолды - компактные пространства, и в этом состоит отличие от пеннеровской конструкции орбифолдов верхней полуплоскости, поскольку в случае конструкции Пеннера все точки редукции имеют групшы стабилизаторов бесконечных порядков.

Обратимся к основной формуле (2.35). Диаграммная техника матричной модели (2.37) сразу дает ответ (при подстановке $\tilde{\Lambda}=e^{\lambda}$ ). Объединяя все члены, получим

$$
F_{1,1}=\alpha^{-1} \frac{3 e^{2 \lambda}-1}{6\left(e^{2 \lambda}-1\right)^{3}}=\frac{1}{48 \alpha} \cdot \frac{\partial^{2}}{\partial \lambda^{2}}\left[\frac{1}{e^{\lambda}-1}-\frac{1}{e^{\lambda}+1}\right]-\frac{1}{12 \alpha}\left[\frac{1}{e^{\lambda}-1}-\frac{1}{e^{\lambda}+1}\right] .
$$

Первый член задает правильное значение $\left\langle\tau_{1}\right\rangle_{1}=1 / 24$. Второй член появляется из "суммы по редукциям", при этом для $\mathcal{M}_{0,3}\left\langle\tau_{0}^{3}\right\rangle_{0}=1$. В сумму по редукциям входит дополнительный дробный множитель $1 / 6$, появляющийся по той причине, что на шесть копий модулярной фигуры $\overline{\mathcal{M}}_{1,1}$ в симплициальном комплексе приходится одна $((1 / 3) \times$ $3=1$ ) копия бесконечной точки. Таким образом, в данном порядке по $g$ и $n$ вклад этой точки в выражение (2.35) равен 1/6.

\section{3. УРАВНЕНИЯ СВЯЗЕЙ В МАТРИЧНЫХ МОДЕЛЯХ}

Все статистические суммы рассматриваемых матричных моделей (в том числе с внешним полем) выражаются через соответствуюшие времена. Для 1ММ это $\left\{\xi_{n}\right\}$ - коэффициенты при степенях матрицы $X$ в потенциале $V(X)$. Для MМК (2.17) набор времен дается всеми нечетными обратными степенями внешней матрицы $\Lambda$ : $t_{n}=(2 n-1) ! ! \operatorname{tr}\left(1 / \Lambda^{2 n+1}\right)$. Наконец, для ММКП имеются два естественных выбора наборов времен. Первый выбор обусловлен структурой ДПМ и два соответствующих симметричных набора времен $\left\{T_{2 n}^{ \pm}\right\}$имеют вид (2.36). Другой выбор связан с интерпретацией модели (2.37) в духе ОМК. В данном разделе эта модель приводится к виду (1.5) с внешним полем $\eta=e^{\lambda}+e^{-\lambda}$; в соответствуюших временах $\xi_{n}=$ $\left(\operatorname{tr} \eta^{-n}\right) / n-\alpha N \delta_{n, 2} / 4$ эта модель оказывается эквивалентной 1ММ.

3.1. Алгебра Вирасоро в 1 ММ. Рассмотрим 1 ММ с произвольным потенциалом:

$$
\mathcal{Z}_{H}\left(\left\{\xi_{m}\right\}, M\right)=\exp \left\{\mathcal{F}_{H}\left(\left\{\xi_{n}\right\}, M\right)\right\} \equiv \int_{M \times M} D Y e^{-V(Y)}, \quad V(Y)=\sum_{n=1}^{\infty} \xi_{n} \operatorname{tr} Y^{n}
$$


где "времена" $\xi_{n}, \quad n \geqslant 1,-$ коэффициенты потенциала, $M$ - размер матрицы $Y$, мера интегрирования $D Y$ имеет вид

$$
D Y \equiv \prod_{i<j}^{M} d \Re Y_{i j} d \Im Y_{i j} \prod_{i=1}^{M} d Y_{i i}
$$

Связи Вирасоро появляются в этой модели из-за инвариантности интеграла относительно произвольных сдвигов переменной интегрирования

$$
Y \rightarrow Y+\varepsilon Y^{n+1}, \quad n \geqslant-1 .
$$

Удерживая члены до первого порядка по $\varepsilon$, получим

$$
\begin{aligned}
\exp \left\{-\sum \xi_{m} \operatorname{tr} Y^{m}\right\} & \rightarrow \exp \left\{-\sum \xi_{m} \operatorname{tr}\left(Y+\varepsilon Y^{n+1}\right)^{m}\right\} \sim \\
& \sim \exp \left\{-\sum \xi_{m} \operatorname{tr} Y^{m}\right\}\left(1-\varepsilon \sum_{m} \xi_{m} \operatorname{tr} m Y^{m+n+1}\right)
\end{aligned}
$$

для экспоненты и

$$
D Y \rightarrow D Y+\varepsilon \prod_{i, j} d Y_{i j} \cdot \sum_{m=0}^{n} Y_{i k}^{m} Y_{l j}^{n-m} \frac{\partial Y_{k l}}{\partial Y_{i j}}=D Y\left(1+\varepsilon \sum_{m=0}^{n} \operatorname{tr} Y^{m} \operatorname{tr} Y^{n-m}\right)
$$

для меры интегрирования. В интеграле можно сделать замену

$$
\operatorname{tr} Y^{m}=-\frac{\partial}{\partial \xi_{m}}, \quad m \geqslant 1, \quad \operatorname{tr} Y^{0}=M \equiv-\frac{\partial}{\partial \xi_{0}}
$$

после чего, варьируя (3.3), получим следующий набор уравнений связи:

$$
\begin{gathered}
L_{n} \mathcal{Z}_{H}\left(\left\{\xi_{m}\right\}, M\right) \equiv\left\{\sum_{m=0}^{n} \frac{\partial}{\partial \xi_{m}} \frac{\partial}{\partial \xi_{n-m}}+\sum_{m=0}^{\infty} m \xi_{m} \frac{\partial}{\partial \xi_{n+m}}\right\} \mathcal{Z}_{H}\left(\left\{\xi_{m}\right\}, M\right)=0 \\
n \geqslant-1
\end{gathered}
$$

где

$$
\frac{\partial}{\partial \xi_{0}} \mathcal{Z}_{H}\left(\left\{\xi_{m}\right\}, M\right) \equiv-M \mathcal{Z}_{H}\left(\left\{\xi_{m}\right\}, M\right)
$$

Операторы $L_{n}$ образуют неаномальную часть алгебры Вирасоро:

$$
\left[L_{n}, L_{m}\right]=(n-m) L_{n+m}, \quad n, m \geqslant-1 .
$$

Уравнения (3.4) и (3.5) полностью задают интеграл (3.1) в асимптотическом разложении по временам. Эти уравнения эквивалентны петлевому уравнению (4.8) (см. ниже), с помощью которого в разделе 4 строится итеративная процедура решения матричных моделей в разложении по $1 / N$. 
Интересно проследить, как меняются условия Вирасоро в родственных моделях, допускающих представление в терминах собственных значений (примерами таких моделей могут служить модель вешественных ортогональных матрищ $O(M)$ и модель симплектических матрищ $S p(2 M))$, в которых матричный интеграл приводится к виду $(2.1)$ с произвольной степенью $\kappa \in \mathbb{R}$ определителя Вандермонда $(\kappa=1$ для матриц $O(M)$ и $\kappa=4$ для матриц из алгебры $S p(2 M))$.

Интеграл

$$
\int \Delta^{\kappa}(x) \exp \left\{-M \sum_{k \geqslant 1} \sum_{i=1}^{M} t_{k} x_{i}^{k}\right\} \prod_{i=1}^{M} d x_{i} \equiv \mathcal{Z}_{\kappa}(t ., M)
$$

удовлетворяет условиям Вирасоро

$$
\left[\frac{\kappa}{2} \frac{1}{M^{2}} \sum_{l=0}^{k-1} \frac{\partial^{2}}{\partial t_{l} \partial t_{k-1-l}}+\frac{\frac{\kappa}{2}-1}{M} \frac{\partial}{\partial t_{k-1}}+\sum_{l \geqslant 1} l t_{l} \frac{\partial}{\partial t_{k+l-1}}\right] \mathcal{Z}_{\kappa}(t ., M)=0
$$

с прежним условием $\partial \mathcal{Z}_{\kappa}(t, M) / \partial t_{0}=-M \cdot \mathcal{Z}_{\kappa}(t,, M)$. В случае, если параметр $\kappa$ не меняется с изменением $M$, имеет место асимптотическое разложение

$$
\mathcal{Z}_{\kappa}(t ., M)=\exp \left\{M^{2} \mathcal{F}_{0}+M^{1} \mathcal{F}_{1 / 2}+\mathcal{F}_{1}+\cdots\right\}
$$

при этом второй член в скобках в формуле (3.7) при всех $\kappa$, кроме $\kappa=2$, разрушает разложение по родам ориентируемых поверхностей (этот факт хорошо известен: например, теории ортогональных матриц отвечают суммам по неориентируемым поверхностям [58]).

3.2. Уравнения Швингера-Дайсона и алгебра Вирасоро в матричной модели Концевича. В интеграле (2.17) сдвинем поле интегрирования на $\Lambda: X \rightarrow X-\Lambda$. Нормировочный множитель

$$
\int D X \exp \left\{-\alpha N \operatorname{tr} \frac{X^{2} \Lambda}{2}\right\}
$$

в представлении $\Lambda=\operatorname{diag}\left(\lambda_{1}, \ldots, \lambda_{N}\right)$ равен $\prod_{i, j=1}^{N}\left(\lambda_{i}+\lambda_{j}\right)^{-1 / 2}$. Таким образом, получим

$$
\mathcal{Z}_{\mathrm{K}}(\{\Lambda\}, \alpha N)=\exp \left\{-\frac{\alpha N}{3} \sum_{j=1}^{N} \lambda_{j}^{3}\right\} \prod_{i, j=1}^{N}\left(\lambda_{i}+\lambda_{j}\right)^{\frac{1}{2}} \widetilde{\mathcal{Z}}_{\mathrm{K}}(\eta)
$$

где $\eta=\Lambda^{2}$ и

$$
\widetilde{\mathcal{Z}}_{\mathrm{K}}(\eta)=\int D X \exp \left\{-\alpha N \operatorname{tr}\left(\frac{X^{3}}{6}-\frac{X \eta}{2}\right)\right\}
$$

Уравнения ШД для интеграла (3.8) получаются варьированием показателя экспоненты по $x_{i j}$. Обозначая через $\langle\cdot\rangle$ усреднение по мере интегрирования и учитывая, что

$$
\left\langle x_{i j}\right\rangle=\frac{\partial}{\partial \eta_{j i}} \widetilde{\mathcal{Z}}_{\mathrm{K}}(\eta),
$$


получим

$$
\left[\left(\frac{2}{\alpha N}\right)^{2} \frac{\partial}{\partial \eta_{i k}} \frac{\partial}{\partial \eta_{k j}}-\eta_{i j}\right] \widetilde{\mathcal{Z}}_{\mathrm{K}}(\eta)=0 .
$$

Используем стандартный прием, позволяюший переписать соотношение (3.9) через собственные значения $\eta_{i}$ матрицы $\eta$. Для этого положим $i=j$ в формуле (3.9). Тогда при каждом фиксированном $j$ действие члена с двумя производными на произвольную функцию, зависящую только от $\operatorname{tr} \eta^{n}, n \in \mathbb{Z}$, эквивалентно оператору

$$
\frac{\partial^{2}}{\partial \eta_{j}^{2}}+\sum_{i \neq j} \frac{1}{\eta_{j}-\eta_{i}}\left(\frac{\partial}{\partial \eta_{j}}-\frac{\partial}{\partial \eta_{i}}\right) .
$$

Подставляя $\left(1 / 2 \lambda_{j}\right)\left(\partial / \partial \lambda_{j}\right)$ вместо $\partial / \partial \eta_{j}$ и используя тождество

$$
\begin{aligned}
& \exp \left\{-\frac{\alpha N}{3} \sum_{j=1}^{N} \lambda_{j}^{3}\right\} \prod_{i, k=1}^{N}\left(\lambda_{i}+\lambda_{k}\right)^{\frac{1}{2}} \frac{\vec{\partial}}{\partial \lambda_{j}}= \\
& =\left[\frac{\vec{\partial}}{\partial \lambda_{j}}+\alpha N \lambda_{j}^{2}-\sum_{i} \frac{1}{\lambda_{i}+\lambda_{j}}\right] \exp \left\{-\frac{\alpha N}{3} \sum_{j=1}^{N} \lambda_{j}^{3}\right\} \prod_{i, k=1}^{N}\left(\lambda_{i}+\lambda_{k}\right)^{\frac{1}{2}} \frac{\vec{\partial}}{\partial \lambda_{j}}
\end{aligned}
$$

получим уравнения ШД для собственно статистической суммы $\mathcal{Z}_{\mathrm{K}}(\{\Lambda\}, \alpha N)$ :

$$
\begin{aligned}
& \left\{\frac{1}{4 \lambda_{j}^{2}}\left(\partial_{j}+\alpha N \lambda_{j}^{2}-\sum_{i} \frac{1}{\lambda_{i+\lambda_{j}}}\right)\left(\partial_{j}+\alpha N \lambda_{j}^{2}-\sum_{k} \frac{1}{\lambda_{k+\lambda_{j}}}\right)+\right. \\
& \quad+\sum_{i \neq j} \frac{1}{\lambda_{j}^{2}-\lambda_{i}^{2}}\left[\frac{1}{2 \lambda_{j}}\left(\partial_{j}+\alpha N \lambda_{j}^{2}-\sum_{k} \frac{1}{\lambda_{k+\lambda_{j}}}\right)-\right. \\
& \left.\quad-\frac{1}{2 \lambda_{i}}\left(\partial_{i}+\alpha N \lambda_{i}^{2}-\sum_{k} \frac{1}{\lambda_{k+\lambda_{i}}}\right)\right]- \\
& \left.\quad-\frac{1}{4 \lambda_{j}^{3}}\left(\partial_{j}+\alpha N \lambda_{j}^{2}-\sum_{i} \frac{1}{\lambda_{i+\lambda_{j}}}\right)-\left(\frac{\alpha N}{2}\right)^{2} \lambda_{j}^{2}\right\} \mathcal{Z}_{\mathrm{K}}(\{\Lambda\}, \alpha N)=0
\end{aligned}
$$

где $\partial_{i} \equiv \partial / \partial \lambda_{i}$. Полезно рассмотреть это уравнение более подробно, что позволит в дальнейшем опустить детали значительно более сложных вычислений в ММКП в параметризации ДПМ.

Объединим сначала члены без производных. Все члены, пропорциональные $\alpha N$, сокрашаются. Оставшаяся часть явно суммируется и в обозначениях

$$
t_{n}=\frac{1}{2 n+1} \operatorname{tr} \frac{1}{\Lambda^{2 n+1}}, \quad t_{n}(j)=\frac{1}{\lambda_{j}^{2 n+2}} \equiv-\partial_{j} t_{n},
$$

в которых

$$
\partial_{j} \rightarrow-\sum_{n=0}^{\infty} \frac{1}{\lambda_{j}^{2 n+2}} \frac{\partial}{\partial t_{n}}
$$


равна

$$
\frac{1}{4} t_{0}(j) t_{0}^{2}+\frac{1}{16} t_{1}(j)
$$

Предположение, что $\mathcal{Z}_{\mathrm{K}}(\{\Lambda\}, \alpha N)$ зависит лишь от следов нечетных степеней внешней матрицы $\Lambda$, приводит к самосогласованному набору уравнений.

Объединяя в (3.10) все члены, содержащие одну производную, получим

$$
-\frac{\alpha N}{2} t_{n}(j) \frac{\partial}{\partial t_{n}}+\sum_{n=0}^{\infty} \frac{\partial}{\partial t_{n}}\left(-\frac{2 n+2}{2 \lambda_{j}^{2 n+5}}+\sum_{i} \frac{1}{2 \lambda_{j}\left(\lambda_{i}+\lambda_{j}\right)} \sum_{m=0}^{2 n+3} \frac{1}{\lambda_{j}^{m} \lambda_{i}^{2 n+3-m}}\right) .
$$

Единственная нетривиальная сумма, содержашая члены $\lambda_{i}+\lambda_{j}$ в знаменателе, оказывается равной

$$
\begin{aligned}
& \frac{1}{2 \lambda_{j}} \sum_{i} \frac{1}{\lambda_{i}+\lambda_{j}} \sum_{m=0}^{2 n+3} \frac{\lambda_{j}^{2 n+3-m} \lambda_{i}^{m}}{\left(\lambda_{i} \lambda_{j}\right)^{2 n+3}}= \\
& \quad=\frac{1}{2 \lambda_{j}} \sum_{i} \sum_{m=0}^{n+1} \frac{\left(\lambda_{i} \lambda_{j}\right)^{m}}{\left(\lambda_{i} \lambda_{j}\right)^{2 n+3}} \frac{\lambda_{j}^{2 n+3-2 m}+\lambda_{i}^{2 n+3-2 m}}{\lambda_{j}+\lambda_{i}}= \\
& \quad=\frac{1}{2} \sum_{m=0}^{n+1} t_{n+1-m}(j)(2 m+1) t_{m},
\end{aligned}
$$

при этом в выражение снова дают вклад только следы нечетных степеней матришы $\Lambda$ (и вариации этих следов по $\lambda_{j}$ ). Окончательный ответ имеет вид

$$
\begin{gathered}
\left\{\frac{1}{4} t_{0}(j) t_{0}^{2}+\frac{1}{16} t_{1}(j)-\frac{\alpha N}{2} \sum_{n=0}^{\infty} t_{n}(j) \frac{\partial}{\partial t_{n}}+\frac{1}{2} \sum_{n=0}^{\infty} \sum_{m=0}^{n+1} t_{n+1-m}(j)(2 m+1) t_{m} \frac{\partial}{\partial t_{n}}+\right. \\
\left.+\frac{1}{4} \sum_{n=0}^{\infty} \sum_{m=0}^{\infty} t_{n+m+2}(j) \frac{\partial}{\partial t_{n}} \frac{\partial}{\partial t_{m}}\right\} \mathcal{Z}_{\mathrm{K}}\left(\left\{t_{n}\right\}, \alpha N\right)=0
\end{gathered}
$$

В асимптотической области больших $N$ все времена $t_{n}(j)$ следует рассматривать как независимые переменные. Тогда уравнение (3.13) примет вид

где операторы

$$
\sum_{n=0}^{\infty} t_{n}(j) L_{n-1} \mathcal{Z}_{\mathrm{K}}\left(\left\{t_{n}\right\}, \alpha N\right)=0
$$

$$
\begin{aligned}
L_{n}= & -\frac{\alpha N}{2} \frac{\partial}{\partial t_{n+1}}+\frac{1}{4} \sum_{m=0}^{n-1} \frac{\partial}{\partial t_{m}} \frac{\partial}{\partial t_{n-1-m}}+ \\
& +\frac{1}{2} \sum_{m=0}^{\infty}(2 m+1) t_{m} \frac{\partial}{\partial t_{n+m}}+\frac{\delta_{n, 0}}{16}, \quad n \geqslant 0 \\
L_{-1}= & -\frac{\alpha N}{2} \frac{\partial}{\partial t_{0}}+\frac{1}{2} \sum_{m=0}^{\infty}(2 m+3) t_{m+1} \frac{\partial}{\partial t_{m}}+\frac{t_{0}^{2}}{4}
\end{aligned}
$$

образуют неаномальную часть алгебры Вирасоро:

$$
\left[L_{n}, L_{m}\right]=(n-m) L_{n+m}, \quad n, m \geqslant-1 .
$$


3.3. Уравнения Швингера-Дайсона для ММКП. Выведем теперь уравнения ШД для статистической суммы ММКП и выразим их через соответствуюшие следы от степеней внешнего матричного поля. Сделаем замену переменных $X \rightarrow$ $-\tilde{\Lambda}^{-1 / 2} X \tilde{\Lambda}^{-1 / 2}-1$ в матричном интеграле (2.37) и приведем интеграл в числителе (2.37) к стандартному виду интеграла ОМК (1.5). Интеграл в знаменателе (2.37) явно вычисляется:

$$
\begin{aligned}
\mathcal{Z}_{\mathrm{KP}}\left(e^{\lambda}\right)= & \frac{(-1)^{-\frac{\alpha N^{2}}{2}}(\operatorname{det} \tilde{\Lambda})^{-N+\frac{\alpha N}{2}} e^{-\frac{\alpha N^{2}}{2}-\frac{\alpha N}{4} \operatorname{tr} \Lambda^{2}}}{\prod_{i, j=1}^{N}\left(\tilde{\Lambda}_{i} \tilde{\Lambda}_{j}-1\right)^{-\frac{1}{2}}\left(\frac{2 \pi}{\alpha N}\right)^{\frac{N 2}{2}}} \times \\
& \times \int D X \exp \left\{-\alpha N \operatorname{tr}\left(\frac{1}{4} X^{2}+\frac{1}{2} \ln X+\frac{1}{2}\left(\tilde{\Lambda}+\tilde{\Lambda}^{-1}\right) X\right)\right\} .
\end{aligned}
$$

Формула (2.3) позволяет проинтегрировать по угловым переменным (несушественные числовые множители опушены):

$$
\begin{aligned}
\mathcal{Z}_{\mathrm{KP}}\left(e^{\lambda}\right)= & \frac{\exp \left\{\left(\frac{\alpha N}{2}-N\right) \sum_{i} \lambda_{i}\right\} \exp \left\{-\frac{\alpha N}{4} \sum_{i} e^{2 \lambda_{i}}\right\}}{\prod_{i=1}^{N}\left(e^{2 \lambda_{i}}-1\right)^{-\frac{1}{2}} \prod_{i<j}^{N}\left(e^{-\lambda_{i}}-e^{-\lambda_{j}}\right)} \times \\
& \times \int \prod_{i=1}^{N} d x_{i} \exp \left\{-\frac{\alpha N}{2} \sum_{i=1}^{N}\left(\frac{1}{2} x_{i}^{2}+\ln x_{i}+\left(e^{\lambda_{i}}+e^{-\lambda_{i}}\right) x_{i}\right)\right\} \prod_{i<j}^{N}\left(x_{i}-x_{j}\right) .
\end{aligned}
$$

Введем обозначение $\eta_{i j}=\left(e^{\lambda}+e^{-\lambda}\right)_{i j}$. Теперь можно явно вывести уравнения ШД для интеграла $(2.37)$ с измененной нормировкой

$$
\mathcal{Z}_{N}\left(e^{\lambda}\right)=\int D X \exp \left\{-\frac{\alpha N}{2} \operatorname{tr}\left[\frac{1}{2} X^{2}+\ln X+X \eta\right]\right\}
$$

через переменные $\eta_{i j}$. Обозначив через $\langle\cdot\rangle$ усреднение с экспоненциальной мерой $(3.18)$, получим

$$
\int D X \frac{\partial}{\partial x_{i j}} \exp \left\{-\frac{\alpha N}{2} \operatorname{tr}\left[\frac{1}{2} X^{2}+\ln X+X \eta\right]\right\}=-\frac{\alpha N}{2}\left\langle x_{i j}+\left[x^{-1}\right]_{i j}+\eta_{i j}\right\rangle=0 .
$$

Принимая во внимание, что

$$
\left\langle x_{i j}\right\rangle=-\frac{2}{\alpha N} \frac{\partial}{\partial \eta_{i j}} \mathcal{Z}_{N}\left(e^{\lambda}\right)
$$

и дифференцируя по $\eta_{j k}$, получим уравнения ШД для $\mathcal{Z}_{N}(\Lambda)$ [54]:

$$
\left(\frac{1}{\left(\frac{\alpha N}{2}\right)^{2}} \frac{\partial}{\partial \eta_{i j}} \frac{\partial}{\partial \eta_{j k}}+\delta_{i k}\left(1-\frac{2}{\alpha}\right)-\frac{2}{\alpha N} \eta_{i j} \frac{\partial}{\partial \eta_{j k}}\right) \mathcal{Z}_{N}\left(e^{\lambda}\right)=0
$$

2 Теоретическая и математическая физика, т. 127, № 2, 2001 г. 
С помошью метода, примененного выше к MMK, сведем (3.20) к уравнению в терминах собственных значений $\eta_{i}$ матрицы $\eta$ :

$$
\left\{\frac{1}{\left(\frac{\alpha N}{2}\right)^{2}}\left[\frac{\partial^{2}}{\partial \eta_{j}^{2}}+\sum_{i \neq j} \frac{\frac{\partial}{\partial \eta_{j}}-\frac{\partial}{\partial \eta_{i}}}{\eta_{j}-\eta_{i}}\right]+\left(1-\frac{2}{\alpha}\right)-\frac{2}{\alpha N} \eta_{j} \frac{\partial}{\partial \eta_{j}}\right\} \mathcal{Z}_{N}\left(e^{\lambda}\right)=0
$$

где

$$
\frac{\partial}{\partial \eta_{i}} \equiv \frac{1}{e^{\lambda_{i}}-e^{-\lambda_{i}}} \frac{\partial}{\partial \lambda_{i}}
$$

Уравнение (3.21) - это основное уравнение ШД для модели (3.18) с логарифмическим потенциалом. Оно имеет два естественных представления через времена: в настояшем разделе показывается, что в подходящей нормировке и при должном выборе времен оно эквивалентно уравнениям 1ММ. В разделе 6 в нормировке (2.37), отвечающей выбору времен $\left\{T_{2 n}^{ \pm}\right\}$, условие (3.21) оказывается связанным с уравнениями ММК.

3.4. Эквивалентность ММКП и 1ММ. Продемонстрируем теперь әквивалентность ММКП и 1ММ двумя способами: в первом сравниваются соответствующие алгебры Вирасоро [62], второй использует явные детерминантные соотношения [44].

Cпособ 1. Условия Вирасоро. Перепишем (3.21) через времена

$$
\xi_{n}=\frac{1}{n} \operatorname{tr} \eta^{-n}-\frac{\alpha N}{4} \delta_{n, 2}, \quad n \geqslant 1, \quad \xi_{0}=-\operatorname{tr} \ln \eta
$$

которые суть преобразование Концевича-Мивы для матрицы $\eta$. С помошью подстановки

$$
\frac{\partial}{\partial \eta_{j}} \rightarrow \sum_{n=0}^{\infty} \frac{\partial}{\partial \xi_{n}}\left(-\frac{1}{\eta_{j}^{n+1}}\right)
$$

получим первый член (3.21):

$$
\frac{\partial^{2}}{\partial \eta_{j}^{2}}+\sum_{i \neq j} \frac{\frac{\partial}{\partial \eta_{j}}-\frac{\partial}{\partial \eta_{i}}}{\eta_{j}-\eta_{i}}=\sum_{n, m=0}^{\infty} \frac{\partial}{\partial \xi_{n}} \frac{\partial}{\partial \xi_{m}} \frac{1}{\eta_{j}^{n+m+2}}+\sum_{n=0}^{\infty} \frac{\partial}{\partial \xi_{n}} \sum_{m=0}^{n} \frac{1}{\eta_{j}^{n-m+1}} \operatorname{tr} \frac{1}{\eta^{m+1}}
$$

Представим $\mathcal{Z}_{N}\left(e^{\lambda}\right)$ в виде

$$
\mathcal{Z}_{N}\left(e^{\lambda}\right)=\exp \left\{\frac{\alpha N}{4} \operatorname{tr} \eta^{2}\right\} \mathcal{Z}_{N}\left(\left\{\xi_{n}\right\}_{n=0}^{\infty}\right) .
$$

Затем, коммутируя экспоненциальный множитель с уравнениями ШДи учитывая сдвиг второго времени $\xi_{2}(3.22)$, получим

$$
\begin{aligned}
& {\left[\sum_{n, m=0}^{\infty} \frac{\partial}{\partial \xi_{n}} \frac{\partial}{\partial \xi_{m}} \frac{1}{\eta_{j}^{n+m+2}}+\right.} \\
& \left.\quad+\sum_{n=0}^{\infty} \sum_{m=0}^{n+1} m \xi_{m} \frac{1}{\eta^{n-m+2}} \frac{\partial}{\partial \xi_{n}}+\left(\frac{\alpha N}{2}\right)^{2}-\frac{\alpha N}{2} \frac{\partial}{\partial \xi_{0}}\right] \mathcal{Z}_{N}\left(\left\{\xi_{n}\right\}\right)=0
\end{aligned}
$$


Собирая теперь вместе все члены, содержащие множитель $\eta_{j}^{-k-2}$, и считая их независимым условием связи $L_{k}$, получим

$$
L_{k} \mathcal{Z}_{N}\left(\left\{\xi_{n}\right\}\right)=\left(\sum_{m=0}^{k} \frac{\partial}{\partial \xi_{m}} \frac{\partial}{\partial \xi_{k-m}}+\sum_{m=0}^{\infty} m \xi_{m} \frac{\partial}{\partial \xi_{k+m}}\right) \mathcal{Z}_{N}\left(\left\{\xi_{n}\right\}\right)=0, \quad k \geqslant-1,
$$

с одним дополнительным ограничением

$$
\frac{\partial}{\partial \xi_{0}} \mathcal{Z}_{N}\left(\left\{\xi_{n}\right\}\right)=\frac{\alpha N}{2} \mathcal{Z}_{N}\left(\left\{\xi_{n}\right\}\right)
$$

Формулы (3.26), (3.27) в точности совпадают с условиями Вирасоро 1ММ (3.4), если мы предположим, что размер соответствуюших эрмитовых матрищ равен $(-\alpha N / 2) \times$ $(-\alpha N / 2)$. Так как формулы $(3.26),(3.27)$ однозначно задают статистическую сумму (3.18) в асимптотическом разложении, то получается точное соотношение между интегралами моделей (3.18) и (3.1):

$$
\mathcal{Z}_{N}\left(e^{\lambda}\right)=\exp \left\{\frac{\alpha N}{4} \operatorname{tr} \eta^{2}\right\} \mathcal{Z}_{H}\left(\left\{\xi_{n}\right\},-\frac{\alpha N}{2}\right),
$$

где времена $\xi_{n}, n \geqslant 0$, связаны с внешней матрицей $\eta=e^{\lambda}+e^{-\lambda}$ преобразованием Концевича-Мивы (3.22).

Спосо6 2. Точные детерминантные соотношения. Другой метод доказательства (3.28) основан на точных соотношениях между детерминантами [44]. Начнем с 1MМ, времена которой удовлетворяют преобразованиям Концевича-Мивы:

$$
\begin{aligned}
\mathcal{Z}_{H}\left(\left\{\xi_{n}\right\}, M\right)= & \int_{M \times M} D Y \exp \left\{-\sum_{n=1}^{\infty}\left(\operatorname{tr}_{(1)} \frac{1}{n} \eta^{-n}+\frac{\delta_{n, 2}}{2}\right) \operatorname{tr}_{(2)} Y^{n}\right\}= \\
= & (2 \pi)^{\frac{M^{2}-M}{2}} \prod_{i=1}^{M}(i !)^{-1} \int \prod_{i=1}^{M} d y_{i} \Delta^{2}(y) \times \\
& \times \exp \left\{-\frac{1}{2} \operatorname{tr}_{(2)} Y^{2}-\sum_{n=1}^{\infty} \operatorname{tr}_{(1)} \eta^{-n} \operatorname{tr}_{(2)} Y^{n}\right\} .
\end{aligned}
$$

Здесь $\operatorname{tr}_{(1)}$ вычисляется по матричным индексам $\eta_{\alpha \beta}, \alpha, \beta=1, \ldots, N$, $\operatorname{atr}_{(2)}-$ по индексам $y_{i j}, i, j=1, \ldots, M$, с последуюшим переходом к интегрированию по собственным значениям (2.1). Второй член в показателе экспоненты может быть представлен в виде

$$
\exp \left\{-\sum_{n=1}^{\infty} \frac{1}{n}\left(\sum_{\alpha=1}^{N} \eta_{\alpha}^{-n}\right)\left(\sum_{i=1}^{M} y_{i}^{-n}\right)\right\}=\prod_{\alpha=1}^{N} \prod_{i=1}^{M} \exp \left\{\ln \left(1-\eta_{\alpha}^{-1} y_{i}\right)\right\}=\prod_{\alpha=1}^{N} \prod_{i=1}^{M} \frac{\eta_{\alpha}-y_{i}}{\eta_{\alpha}}
$$

Объединим теперь переменные $\left\{y_{i}\right\}$ и $\left\{\eta_{\alpha}\right\}$ в один набор $\left\{z_{k}\right\}, k=1, \ldots, M+N$, где первые $M$ переменных суть $y_{i}$, а последние $N$ суть $\eta_{\alpha}$. Заметим, что

$$
\Delta(y) \prod_{\alpha=1}^{N} \prod_{i=1}^{M}\left(\eta_{\alpha}-y_{i}\right)=\frac{\Delta(z)}{\Delta(\eta)}
$$


где $\Delta(z)$ - полный детерминант Вандермонда. Теперь интеграл (3.29) примет вид

$$
(\operatorname{det} \eta)^{-M} \int \prod_{i=1}^{M} d y_{i} \Delta(y) \frac{\Delta(z)}{\Delta(\eta)} \exp \left\{-\frac{1}{2} \sum_{i=1}^{M} y_{i}^{2}\right\}
$$

и можно проинтегрировать по переменным $y_{i}$. Детерминант $\Delta(z)$ допускает представление

$$
\begin{aligned}
& \Delta(z)=\operatorname{det}\left|\begin{array}{cccccc}
1 & \ldots & 1 & 1 & \ldots & 1 \\
y_{1} & \ldots & y_{M} & \eta_{1} & \ldots & \eta_{N} \\
y_{1}^{2} & \ldots & y_{M}^{2} & \eta_{1}^{2} & \ldots & \eta_{N}^{2} \\
\vdots & & \vdots & \vdots & & \vdots \\
y_{1}^{M+N+1} & \ldots & y_{M}^{M+N+1} & \eta_{1}^{M+N+1} & \ldots & \eta_{N}^{M+N+1}
\end{array}\right| \equiv \\
& \equiv \operatorname{det}\left|\begin{array}{cccccc}
1 & \ldots & 1 & 1 & \ldots & 1 \\
P_{1}\left(y_{1}\right) & \ldots & P_{1}\left(y_{M}\right) & P_{1}\left(\eta_{1}\right) & \ldots & P_{1}\left(\eta_{N}\right) \\
P_{2}\left(y_{1}\right) & \ldots & P_{2}\left(y_{M}\right) & P_{2}\left(\eta_{1}\right) & \ldots & P_{2}\left(\eta_{N}\right) \\
\vdots & & \vdots & \vdots & & \vdots \\
P_{M+N-1}\left(y_{1}\right) & \ldots & P_{M+N-1}\left(y_{M}\right) & P_{M+N-1}\left(\eta_{1}\right) & \ldots & P_{M+N-1}\left(\eta_{N}\right)
\end{array}\right|
\end{aligned}
$$

которое справедливо для любых полиномов $P_{k}(z)=z^{k}+a_{k-1}^{(k)} z^{k-1}+\cdots+a_{0}^{(k)}$. Выберем $P_{k}(z)$ в виде полиномов Эрмита $H_{k}(z)$ :

$$
\int_{-\infty}^{\infty} H_{n}(z) H_{m}(z) e^{-\frac{z^{2}}{2}} d z \equiv\left\langle H_{n} \mid H_{m}\right\rangle=h_{n} \delta_{n, m}
$$

Представляя $\Delta(y)$ в виде $(3.30)$ и умножая его на $\Delta(z)$, получим

$$
\begin{gathered}
(\operatorname{det} \eta)^{-M} \int \prod_{i=1}^{M} d y_{i} \frac{1}{\Delta(\eta)}\left(\sum_{\left\{i_{k}\right\}} \varepsilon\left(\left\{i_{k}\right\}\right) \prod_{k=1}^{M} H_{i_{k}-1}\left(y_{k}\right)\right) \times \\
\times\left(\sum_{\left\{j_{s}\right\}} \varepsilon\left(\left\{j_{s}\right\}\right) \prod_{s=1}^{M+N} H_{j_{s}-1}\left(z_{s}\right)\right) \exp \left\{-\sum_{k=1}^{M} \frac{y_{k}^{2}}{2}\right\} .
\end{gathered}
$$

Здесь $\left\{i_{k}\right\},\left\{j_{s}\right\}$ - перестановки индексов, $\varepsilon\left(\left\{i_{k}\right\}\right), \varepsilon\left(\left\{j_{s}\right\}\right)$ равны \pm 1 в зависимости от четности перестановки. Единственный ненулевой вклад в (3.32) мыполучим при $i_{k}=j_{k}$ для $k=1,2, \ldots, M$. В противном случае в правом сомножителе всегда найдется член $H_{r}\left(y_{i}\right)$ c $r>M-1$, который обратится в нуль при усреднении с мерой $e^{-y_{i}^{2} / 2}$. В результате получим

$$
(\operatorname{det} \eta)^{-M} \frac{1}{\Delta(\eta)} M ! \prod_{k=1}^{M} h_{k-1} \sum_{\left\{j_{\alpha}\right\}} \varepsilon\left(\left\{j_{\alpha}\right\}\right) \prod_{\alpha=1}^{N} H_{j_{\alpha}+M-1}\left(\eta_{\alpha}\right),
$$

где $\left\{j_{\alpha}\right\}, \alpha=1, \ldots, N,-$ перестановки индексов $1, \ldots, N$. Соотношение (3.33) снова может рассматриваться как отношение двух детерминантов. 
Далее, полиномы Эрмита $H_{n}(\eta)$ допускают представление

$$
H_{n}(\eta)=\frac{e^{\frac{\eta^{2}}{2}}}{\sqrt{2 \pi}}(-i)^{n} \int_{-\infty}^{\infty} x^{n} e^{-\frac{x^{2}}{2}+i x \eta} d x, \quad h_{n}=\sqrt{2 \pi} n !
$$

В выражении (3.33) номера полиномов Эрмита больше или равны $M-1$. Следовательно, в выражении (3.34) степень монома, стояшего перед экспонентой, должна быть равна $M+j_{\alpha}-1$, т.е. можно вынести обший множитель $x_{\alpha}^{M}$ :

$$
\begin{aligned}
\mathcal{Z}_{H}\left(\left\{\xi_{n}\right\}, M\right) & =(2 \pi)^{\frac{M^{2}-N}{2}}(\operatorname{det} \eta)^{-M} e^{\frac{\operatorname{tr} \eta^{2}}{2}} \times \\
\times & \int_{-\infty}^{\infty} \prod_{\alpha=1}^{N} d x_{\alpha} \frac{\Delta(x)}{\Delta(i \eta)} \exp \left\{\sum_{\alpha=1}^{N}\left(-\frac{x_{\alpha}^{2}}{2}+i x_{\alpha} \eta_{\alpha}+M \ln x_{\alpha}\right)\right\} .
\end{aligned}
$$

Этот интеграл уже имеет вид (2.3) с потенциалом $-X^{2} / 2+i \eta X+M \ln X$ :

$$
\begin{aligned}
\mathcal{Z}_{H}\left(\left\{\xi_{n}\right\}, M\right)= & (2 \pi)^{\frac{M^{2}-N^{2}}{2}}(\operatorname{det} \eta)^{-M} e^{\frac{\operatorname{tr} \eta^{2}}{2}} \times \\
& \times \int_{N \times N} D X \exp \left\{\operatorname{tr}\left[-\frac{X^{2}}{2}+i X \eta+M \ln X\right]\right\},
\end{aligned}
$$

и он эквивалентен (3.28) при замене $\eta \rightarrow i \eta$.

Из (3.28) видно, что необходимо сравнивать ММКП с 1ММ с “отрицательным” размером матриц, $M=-\alpha N / 2$, для того чтобы обеспечить сходимость интеграла (3.18). Обратимся к диаграммной технике 1ММ. Размер матрицы интегрирования входит в ответ через множители, стоящие при каждой грани триангулируемой поверхности (см. рис. 2). Множитель $M$ отвечает каждой замкнутой линии индекса, бегушего по границе грани. Эйлерова характеристика ориентированной замкнутой поверхности равна

$$
\# X_{2}-\# X_{1}+\# X_{0}=2-2 g,
$$

где $g$ - род поверхности, а \# $X_{i}$ - число симплексов размерности $i$. С одной стороны,

$$
\# X_{1}=\frac{1}{2} \sum_{i \in X_{0}} v_{i}
$$

где $v_{i}$ - валентности вершин. Тогда

$$
\# X_{2}=2-2 g+\frac{1}{2} \sum_{i \in X_{0}}\left(v_{i}-2\right) \text {. }
$$

При изменении знака $M$ появляется множитель

$$
(-1)^{\# X_{2}}=(-1)^{\frac{1}{2} \sum_{i \in X_{0}}\left(v_{i}-2\right)}=\prod_{i \in X_{0}}(-1)(i)^{v_{i}} .
$$

Этот множитель отвечает изменению знака при потенциале с одновременным “виковским поворотом" внешнего матричного поля $\eta \rightarrow i \eta$ в формулах (3.22). Это в точности соответствует переходу от (3.28) к (3.36). 


\section{4. ВЫЧИСЛЕНИЯ В МАТРИЧНЫХ МОДЕЛЯХ В РАЗЛОЖЕНИИ ПО РОДАМ}

4.1. Петлевые средние в 1 ММ. Средние, отвечаюшие статистической сумме $1 \mathrm{MM} \mathrm{(2.1),} \mathrm{определяются} \mathrm{обычным} \mathrm{образом:}$

$$
\langle Q(X)\rangle=\frac{1}{Z} \int_{M \times M} D X \exp (-M \operatorname{tr} V(X)) Q(X) .
$$

Производяший функционал (однопетлевое среднее)

$$
W(p)=\frac{1}{M} \sum_{k=0}^{\infty} \frac{\left\langle\operatorname{tr} X^{k}\right\rangle}{p^{k+1}}
$$

и $s$-петлевой коррелятор $(s \geqslant 2)$

$$
W\left(p_{1}, \ldots, p_{s}\right)=M^{s-2} \sum_{k_{1}, \ldots, k_{s}=1}^{\infty} \frac{\left\langle\operatorname{tr} X^{k_{1}} \ldots \operatorname{tr} X^{k_{s}}\right\rangle_{\mathrm{conn}}}{p_{1}^{k_{1}+1} \ldots p_{s}^{k_{s}+1}},
$$

где индекс "conn" обозначает связную часть, могут быть переписаны в виде

$$
W\left(p_{1}, \ldots, p_{s}\right)=M^{s-2}\left\langle\operatorname{tr} \frac{1}{p_{1}-X} \ldots \operatorname{tr} \frac{1}{p_{s}-X}\right\rangle_{\text {conn }} .
$$

Как показано в работе [79], эти величины связаны с преобразованиями Лапласа сумм по дискретизованным открытым поверхностям с $s$-компонентной границей.

Корреляторы получаются из функционала свободной энергии $F$ с помощью оператора вставки петли $d / d V(p)$ :

$$
W\left(p_{1}, \ldots, p_{s}\right)=\frac{d}{d V\left(p_{s}\right)} \frac{d}{d V\left(p_{s-1}\right)} \ldots \frac{d F}{d V\left(p_{1}\right)}=\frac{d}{d V\left(p_{s}\right)} \frac{d}{d V\left(p_{s-1}\right)} \ldots \frac{d}{d V\left(p_{2}\right)} W\left(p_{1}\right),
$$

где

$$
\frac{d}{d V(p)} \equiv-\sum_{j=1}^{\infty} \frac{1}{p^{j+1}} \frac{d}{d \xi_{j}}
$$

Таким образом, если однопетлевой коррелятор известен для произвольного потенциала, то можно вычислить все многопетлевые корреляторы.

В нормировке, приведенной вьше, разложение по родам имеет вид

$$
W\left(p_{1}, \ldots, p_{s}\right)=\sum_{g=0}^{\infty} \frac{1}{M^{2 g}} W_{g}\left(p_{1}, \ldots, p_{s}\right), \quad s \geqslant 1,
$$

аналогичный разложению по родам для свободной энергии

$$
F=\sum_{g=0}^{\infty} \frac{1}{M^{2 g}} F_{g} .
$$


4.1.1. Петлевое уравнение. Первое из цепочки петлевых уравнений $1 \mathrm{MM}[48]$ легко получить, суммируя условия Вирасоро $L_{n}(3.4)$ с весом $p^{-n-2}$ :

$$
\oint_{C} \frac{d \omega}{2 \pi i} \frac{V^{\prime}(\omega)}{p-\omega} W(\omega)=(W(p))^{2}+\frac{1}{M^{2}} W(p, p) .
$$

Здесь $C$ - кривая, окружающая сингулярные точки $W(\omega)$, но не точку $\omega=p$. Это контурное интегрирование действует как проектор, выделяющий коэффициент при члене $p^{-1}$. Второй член в правой части петлевого уравнения (4.8) с помошью уравнения (4.5) может быть выражен через $W(p)$, так что (4.8) - замкнутое уравнение, задающее эту величину.

В ведущем порядке по $1 / M^{2}$ можно опустить последний член в (4.8) (что отвечает бездисперсионному пределу в иерархии уравнений цепочки Тоды), предположить, что сингулярность $W(\omega)$ сосредоточена на единственном разрезе на вешественной оси, и учесть, что $W(p) \sim 1 / p$ при $p \rightarrow \infty$. В этих предположениях решение имеет вид [80]

$$
W_{0}(p)=\frac{1}{2} \oint_{C} \frac{d \omega}{2 \pi i} \frac{V^{\prime}(\omega)}{p-\omega}\left\{\frac{(p-x)(p-y)}{(\omega-x)(\omega-y)}\right\}^{\frac{1}{2}}
$$

где $x$ и $y$ задаются потенциалом матричной модели:

$$
\oint_{C} \frac{d \omega}{2 \pi i} \frac{V^{\prime}(\omega)}{\sqrt{(\omega-x)(\omega-y)}}=0, \quad \oint_{C} \frac{d \omega}{2 \pi i} \frac{\omega V^{\prime}(\omega)}{\sqrt{(\omega-x)(\omega-y)}}=2 .
$$

Это решение для рода ноль используется ниже при решении петлевого уравнения по теории возмушений.

При подстановке разложения по родам (4.7) в уравнение $(4.8)$ получим, что $W_{g}(p)$ при $g \geqslant 1$ удовлетворяет уравнению

$$
\left\{\widehat{K}-2 W_{0}(p)\right\} W_{g}(p)=\sum_{g^{\prime}=1}^{g-1} W_{g^{\prime}}(p) W_{g-g^{\prime}}(p)+\frac{d}{d V(p)} W_{g-1}(p),
$$

где $\widehat{K}$ - линейный оператор,

$$
\widehat{K} f(p) \equiv \oint_{C} \frac{d \omega}{2 \pi i} \frac{V^{\prime}(\omega)}{p-\omega} f(\omega)
$$

В уравнении (4.11) $W_{g}(p)$ выражается только через такие $W_{g_{i}}(p)$, для которых $g_{i}<g$. Именно это позволяет развить итеративную процедуру.

4.1.2. Моменты модели. Введем вместо старых констант связи $\xi_{j}$ новые переменные (моменты) $M_{k}$ и $J_{k}$, которые будут характеризовать потенциал матричной модели:

$$
\begin{aligned}
M_{k} & =\oint_{C} \frac{d \omega}{2 \pi i} \frac{V^{\prime}(\omega)}{(\omega-x)^{k+\frac{1}{2}}(\omega-y)^{\frac{1}{2}}}, \quad k \geqslant 1, \\
J_{k} & =\oint_{C} \frac{d \omega}{2 \pi i} \frac{V^{\prime}(\omega)}{(\omega-x)^{\frac{1}{2}}(\omega-y)^{k+\frac{1}{2}}}, \quad k \geqslant 1 .
\end{aligned}
$$


Эти моменты зависят от констант связи $\xi_{j}$ как явно, так и через параметры $x$ и $y$, определяемые уравнениями (4.10), причем $M_{k}$ и $J_{k}$ явно зависят только от $\xi_{j}$ с $j \geqslant k$.

Ниже будет показано, что в разложении по родам для свободной энергии и корреляторов сложные выражения, зависящие от бесконечного числа констант связи, оказываются рациональными функциями, зависящими от конечного числа моментов в каждом роде. Кроме того, критическое поведение $1 \mathrm{MM} \mathrm{получается} \mathrm{из} \mathrm{моментов} \mathrm{более} \mathrm{нагля} \mathrm{дно,}$ чем из констант связи ${ }^{5)}$.

Вычисляя контурный интеграл в (4.9) с помощью взятия вычетов в точках $\omega=p$ и $\omega=\infty$, найдем

$$
W_{0}(p)=\frac{1}{2}\left\{V^{\prime}(p)-M(p) \sqrt{(p-x)(p-y)}\right\},
$$

где $M(p)$ - полином от $p$, степень которого на два меньше, чем степень $V(p)$. Кроме того, $W_{0}(p)$ можно найти, анализируя матричную модель с использованием метода собственных значений [15]. Условие аналитичности $W_{0}(p)$ в комплексной плоскости всюду, кроме разреза $[y, x]$ на вещественной оси, и асимптотическое поведение петлевого среднего $W_{0}(p) \sim 1 / p$ при $p \rightarrow \infty$ отвечают требованию, чтобы плотность собственных чисел $\rho(\lambda)$, определяемая скачком мнимой части $W_{0}(p)$ на разрезе, имела бы носитель на отрезке $[y, x]$ и была бы нормирована на единицу. Из (4.15) следует, что плотность собственных значений имеет вид

$$
\rho(\lambda)=\frac{1}{\pi} M(\lambda) \sqrt{(\lambda-y)(x-\lambda)}, \quad y \leqslant \lambda \leqslant x .
$$

В случае потенциала $V(X)$ обшего положения эта функция убывает, как квадратный корень, на обоих концах своей области определения. Критическому поведению отвечает случай, когда один из корней $M(\lambda)$ стремится к $x$ или $y$. В несимметричном потенциале $m$-я мультикритическая точка отвечает случаю, когда на одном из концов $-x$ или $y-$ собирается $(m-1)$ дополнительных нулей. Сравнивая $(4.9)$ и $(4.15)$, получим, что

$$
\left.M_{k} \propto \frac{d^{(k-1)} M(\lambda)}{d \lambda^{k-1}}\right|_{\lambda=x},\left.\quad J_{k} \propto \frac{d^{(k-1)} M(\lambda)}{d \lambda^{k-1}}\right|_{\lambda=y},
$$

и тем самым условие $m$-й мультикритической точки просто имеет вид $M_{1}=M_{2}=\cdots$ $\cdots=M_{k-1}=0$ и $M_{k} \neq 0$, если дополнительные нули собираются в точке $x$, и $J_{1}=$ $J_{2}=\cdots=J_{k-1}=0, J_{k} \neq 0$, если дополнительные нули собираются в точке $y$. В симметричном потенциале $(V(X)=V(-X))$ собственные значения распределяются также симметрично относительно нуля. В этом случае $m$-я мультикритическая точка задается плотностью собственных значений, имеющей $(m-1)$ дополнительных нулей в обеих концевых точках интервала распределения собственных значений. В случае симметричного потенциала $M_{k}=(-1)^{k+1} J_{k}$, а условие попадания в $m$-ю мультикритическую точку сводится к обрашению в нуль $(m-1)$ младших моментов.

\footnotetext{
5) Заметим, однако, что в отличие от ММК, в которой соответствующие моменты $I_{n}$ можно считать новыми временами, в $1 \mathrm{MM}$ нет взаимно однозначного соответствия между $\left\{\xi_{k}\right\}$ и $M_{k}, J_{k}$. "Удвоение" времен происходит только в подходе ДПМ.
} 
Аналогично можно рассмотреть и более общую ситуацию, когда $m_{1}$ дополнительных нулей собираются в точке $x$ и $m_{2}$ нулей - в точке $y$. Такие мультикритические модели исследовались в работах [81]. Также можно рассмотреть случай, когда носитель множества собственных значений $\rho$ разбивается на два или более интервалов, при этом петлевое среднее имеет корневые особенности в более чем двух точках на вещественной прямой. В случае ММКП (эквивалентном 1ММ) такие (многоразрезные) решения рассматривались в работе [82], где было показано, что в этом случае также развивается мультикритическое поведение, сходное с мультикритическим поведением в технике моментов.

\section{2. Процедура итераций.}

4.2.1. Структура $F_{g}$ и $W_{g}(p)$. Покажем, что итеративная процедура решения петлевого уравнения приводит к следующему представлению для вклада рода $g$ в свободную энергию:

$$
F_{g}=\sum_{\substack{\alpha_{j}>1 \\ \beta_{i}>1}}\left\langle\alpha_{1} \ldots \alpha_{s} ; \beta_{1} \ldots \beta_{l} \mid \alpha, \beta, \gamma\right\rangle_{g} \frac{M_{\alpha_{1}} \ldots M_{\alpha_{s}} J_{\beta_{1}} \ldots J_{\beta_{l}}}{M_{1}^{\alpha} J_{1}^{\beta} d^{\gamma}}, \quad g \geqslant 1,
$$

где $d \equiv x-y$-расстояние между концами области распределения собственных значений, а скобки суть рациональные числа, в то время как $\alpha, \beta$ и $\gamma$-неотрицательные целые числа. Индексы $\alpha_{1}, \ldots, \alpha_{s}, \beta_{1}, \ldots, \beta_{l}$ принимают значения в интервале $[2,3 g-2]$ и суммирование ведется по всем наборам индексов. В частности, величина $F_{g}$ зависит не более чем от $2(3 g-2)$ различных моментов. Более того, поскольку нет никакого различия между точками $x$ и $y$, свободная энергия $F_{g}$ должна быть инвариантна относительно их перестановки. Таким образом, получим

$$
F:\left\langle\alpha_{1} \ldots \alpha_{s} ; \beta_{1} \ldots \beta_{l} \mid \alpha, \beta, \gamma\right\rangle=(-1)^{\gamma}\left\langle\beta_{1} \ldots \beta_{l} ; \alpha_{1} \ldots \alpha_{s} \mid \beta, \alpha, \gamma\right\rangle
$$

На целые числа, входящие в формулу (4.18), возникает целый ряд ограничений. Обозначим через $N_{M}$ и $N_{J}$ соответствующие полные степени моментов $M_{k}$ и $J_{k}$ :

$$
N_{M}=s-\alpha, \quad N_{J}=l-\beta
$$

Тогда имеют место соотношения

$$
N_{M} \leqslant 0, \quad N_{J} \leqslant 0
$$

и

$$
\begin{aligned}
& F_{g}: N_{M}+N_{J}=2-2 g \\
& F_{g}: \sum_{i=1}^{s}\left(\alpha_{i}-1\right)+\sum_{j=1}^{l}\left(\beta_{j}-1\right)+\gamma=4 g-4 .
\end{aligned}
$$


Соотношение (4.22) следует из инвариантности статистической суммы $Z=$ $\exp \left\{\sum_{g} M^{2-2 g} F_{g}\right\}$ при одновременном изменении размера матриш $M$ и плотности собственных значений $\rho(\lambda): M \rightarrow k M, \rho(\lambda) \rightarrow \rho(\lambda) / k$. Соотношение (4.23) следует из инвариантности $Z$ при масштабных преобразованиях вида $M \rightarrow k^{2} M, \xi_{j} \rightarrow k^{j-2} \xi_{j}$. Наконец, должно выполняться следующее неравенство:

$$
F_{g}: \sum_{i=1}^{s}\left(\alpha_{i}-1\right)+\sum_{j=1}^{l}\left(\beta_{j}-1\right) \leqslant 3 g-3 .
$$

Это требование следует из ДСП (см. ниже). В совокупности с соотношением (4.23) оно дает ограничение

$$
g-1 \leqslant \gamma \leqslant 4 g-4
$$

Чтобы выяснить структуру $W_{g}(p)$, введем базисные векторы $\chi^{(n)}(p)$ и $\Psi^{(n)}(p)$ :

$$
\begin{array}{ll}
\chi^{(n)}(p)=\frac{1}{M_{1}}\left\{\Phi_{x}^{(n)}(p)-\sum_{k=1}^{n-1} \chi^{(k)}(p) M_{n-k+1}\right\}, & n \geqslant 1 \\
\Psi^{(n)}(p)=\frac{1}{J_{1}}\left\{\Phi_{y}^{(n)}(p)-\sum_{k=1}^{n-1} \Psi^{(k)}(p) J_{n-k+1}\right\}, & n \geqslant 1
\end{array}
$$

где

$$
\begin{array}{ll}
\Phi_{x}^{(n)}(p)=(p-x)^{-n}\{(p-x)(p-y)\}^{-\frac{1}{2}}, & n \geqslant 0, \\
\Phi_{y}^{(n)}(p)=(p-y)^{-n}\{(p-x)(p-y)\}^{-\frac{1}{2}}, \quad n \geqslant 0 .
\end{array}
$$

Как легко показать, для оператора $\widehat{K}$, заданного формулой (4.12), справедливы соотношения

$$
\begin{aligned}
& \left\{\widehat{K}-2 W_{0}(p)\right\} \chi^{(n)}(p)=\frac{1}{(p-x)^{n}}, \quad n \geqslant 1, \\
& \left\{\widehat{K}-2 W_{0}(p)\right\} \Psi^{(n)}(p)=\frac{1}{(p-y)^{n}}, \quad n \geqslant 1,
\end{aligned}
$$

а ядро оператора $\left\{\widehat{K}-2 W_{0}(p)\right\}$ заметается функциями $\Phi_{x}^{(0)}(p)=\Phi_{y}^{(0)}(p)$.

Так как $W_{g}(p)$ - вклад рода $g$ в однопетлевой коррелятор - может быть получен из $F_{g}$ с помощюю уравнения (4.5), то из представления (4.18) следует, что $\left.{ }^{6}\right)$

$$
W_{g}(p)=\sum_{n=1}^{3 g-1}\left\{A_{g}^{(n)} \chi^{(n)}(p)+D_{g}^{(n)} \Psi^{(n)}(p)\right\}, \quad g \geqslant 1
$$

\footnotetext{
6) Эквивалентная формула доказана в работе [60] при несколько отличных определениях моментов и базисных векторов.
} 
Заметим, что добавление слагаемых, содержащих $\Phi_{x}^{(0)}(p)$ или $\Phi_{y}^{(0)}(p)$, противоречит граничному условию $W(p) \rightarrow 1 / p$ при $p \rightarrow \infty$, так как это граничное условие было уже учтено при вычислениях для рода ноль. Предложенная структура $W_{g}(p)$ согласуется с предположением [83], что функция $W_{g}(p)$ аналитична в комплексной плоскости всюду, за исключением разреза $[y, x]$ на вешественной оси.

Коэффициенты $A_{g}^{(n)}$ имеют ту же структуру, что и $F_{g}$, и соотношения (4.21) по-прежнему справедливы. Однако в этом случае индексы $\alpha_{1}, \ldots, \alpha_{s}, \beta_{1}, \ldots, \beta_{l}$ принимают значения в интервале $[2,3 g-n]$. Следовательно, $W_{g}(p)$ зависит не более чем от $2(3 g-1)$ моментов. Отмеченная вьше инвариантность статистической суммы при масштабных преобразованиях налагает следующие ограничения на структуру $A_{g}^{(n)}$ :

$$
\begin{array}{ll}
A_{g}^{(n)}: & N_{M}+N_{J}=2-2 g, \\
A_{g}^{(n)}: & \sum_{i=1}^{s}\left(\alpha_{i}-1\right)+\sum_{j=1}^{l}\left(\beta_{j}-1\right)+\gamma=4 g-2-n .
\end{array}
$$

Можно также получить аналог соотношения (4.24) для $A_{g}^{(n)}$ :

$$
A_{g}^{(n)}: \sum_{i=1}^{k}\left(\alpha_{i}-1\right)+\sum_{j=1}^{l}\left(\beta_{j}-1\right) \leqslant 3 g-n-1 .
$$

Объединяя (4.34) и (4.35), можно опять получить ограничение (4.25) на $\gamma$.

Как и $F_{g}$, коррелятор $W_{g}(p)$ должен быть инвариантен при перестановке $x$ и $y$. Поэтому $D_{g}^{(n)}$ переходит в $A_{g}^{(n)}$ при перестановках $d \rightarrow-d, J_{k} \leftrightarrow M_{k}$. (Заметим, что аналог соотношения (4.19) для $A_{g}^{(n)}$ отсутствует.) В случае симметричного потенциала $W_{g}(p)$ есть нечетная функция и $A_{g}^{(n)}=(-1)^{n} D_{g}^{(n)}$.

Доказательство того, что структуры $F_{g}$ и $W_{g}(p)$ даются соответственно формулами (4.18) и (4.32), может быть проведено по индукции (см. [60], [84]). В работе [84] формула, аналогичная (4.32), была доказана в случае комплексной матричной модели. Стратегия доказательства будет ясна, если обратиться к п. 4.2.2 и 4.2.3, в которых описывается итеративная процедура, позволяюшая вычислить $W_{g}(p)$ и $F_{g}$ для произвольного рода $g$, если известен $W_{0}(p)$ - ответ для рода ноль.

4.2.2. Итеративная процедура для определения $W_{g}(p)$. В соответствии с (4.8) итеративная процедура начинается с нахождения $W_{0}(p, p)$. Представим оператор вставки петли в виде

$$
\frac{d}{d V(p)}=\frac{\partial}{\partial V(p)}+\frac{d x}{d V(p)} \frac{\partial}{\partial x}+\frac{d y}{d V(p)} \frac{\partial}{\partial y},
$$

где $\partial / \partial V(p)$ не действует на функционалы $x$ и $y$. Производные $d x / d V(p)$ и $d y / d V(p)$ можно получить из (4.10):

$$
\frac{d x}{d V(p)}=\frac{1}{M_{1}} \Phi_{x}^{(1)}, \quad \frac{d y}{d V(p)}=\frac{1}{J_{1}} \Phi_{y}^{(1)} .
$$


Используя соотношение

$$
\frac{\partial}{\partial V(p)} V^{\prime}(\omega)=\frac{\partial}{\partial p} \frac{1}{p-\omega}
$$

получим [33]

$$
W_{0}(p, p)=\frac{(x-y)^{2}}{16(p-x)^{2}(p-y)^{2}}
$$

Это позволяет найти петлевую поправку $W_{1}(p)$, которая имеет вид $(4.32)$ с

$$
\begin{array}{ll}
A_{1}^{(1)}=-\frac{1}{8 d}, & A_{1}^{(2)}=\frac{1}{16}, \\
D_{1}^{(1)}=\frac{1}{8 d}, & D_{1}^{(2)}=\frac{1}{16} .
\end{array}
$$

Теперь можно начинать процесс итераций. На каждом шаге надо вычислить правую часть петлевого уравнения (4.8). Разлагая полученный результат по дробям вида $(p-x)^{-n},(p-y)^{-n}$, можно сразу идентифицировать коэффициенты $A_{g}^{(n)}$ и $D_{g}^{(n)}$.

Чтобы вычислить $W_{g}(p, p)$, удобно переписать оператор вставки петли в виде

$$
\frac{d}{d V(p)}=\sum_{n} \frac{d M_{n}}{d V(p)} \frac{\partial}{\partial M_{n}}+\sum_{j} \frac{d J_{j}}{d V(p)} \frac{\partial}{\partial J_{j}}+\frac{d x}{d V(p)} \frac{\partial}{\partial x}+\frac{d y}{d V(p)} \frac{\partial}{\partial y}
$$

где

$$
\begin{aligned}
\frac{d M_{n}}{d V(p)}= & -\frac{1}{2}(p-x)^{-n-\frac{1}{2}}(p-y)^{-\frac{3}{2}}-\left(n+\frac{1}{2}\right) \Phi_{x}^{(n+1)}(p)+ \\
& +\frac{1}{2}\left\{\sum_{i=1}^{n}(-1)^{n-i} M_{i}\left(\frac{1}{x-y}\right)^{n-i+1}+(-1)^{n} J_{1}\left(\frac{1}{x-y}\right)^{n}\right\} \frac{d y}{d V(p)}+ \\
& +\left(n+\frac{1}{2}\right) M_{n+1} \frac{d x}{d V(p)}
\end{aligned}
$$

Производные $d x / d V(p)$ и $d y / d V(p)$ даются формулами (4.37), а функция $\Phi_{x}^{(n)}$ была получена в (4.28). Производные $d J_{n} / d V(p)$ можно получить из производных $d M_{n} / d V(p)$ при заменах $y \leftrightarrow x$ и $J_{k} \leftrightarrow M_{k}$. Заметим, что в случае симметричного потенциала алгоритм не упрошается и положить $x=-y$ можно лишш в самом конце выгислений.

Изучая оператор вставки петли (4.42) с учетом формул (4.39)-(4.41), найдем, что $A_{g}^{(n)}$ и $D_{g}^{(n)}$ зависят от $x$ и $y$ только через комбинацию $(x-y)$ и тем самым обладают структурой, приведенной в формуле (4.18). Более того, оказывается, что $W_{g}\left(p_{1}, \ldots, p_{s}\right)$ зависит от не более чем $2(3 g-2+s)$ параметров. Результат для рода два был получен с помощью программы Mathematica в работе [53]. Он слишком громоздок, чтобы быть приведенным здесь. Отметим лишь, что ненулевыми коэффициентами в нем являются $A_{2}^{(1)}-A_{2}^{(5)}$ и $D_{2}^{(1)}-D_{2}^{(5)}$. Вклад рода два в $W(p)$ задается формулой $(4.32)$. 
4.2.3. Итеративная процедура для $F_{g}$. В этом пункте предлагается алгоритм, позволяющий определить $F_{g}$ исходя из известного ответа для $W_{g}(p)$. Метод состоит в том, чтобы представить базисные векторы $\chi^{(n)}(p)$ и $\Psi^{(n)}(p)$ как производные по $V(p)$. Нетрудно проверить, что выполняются следуюшие соотношения:

$$
\begin{aligned}
\chi^{(1)}(p) & =\frac{d x}{d V(p)}, \\
\Psi^{(1)}(p) & =\frac{d y}{d V(p)}, \\
\chi^{(2)}(p) & =\frac{d}{d V(p)}\left\{-\frac{2}{3} \ln M_{1}-\frac{1}{3} \ln d\right\}, \\
\Psi^{(2)}(p) & =\frac{d}{d V(p)}\left\{-\frac{2}{3} \ln J_{1}-\frac{1}{3} \ln d\right\} .
\end{aligned}
$$

Объединяя эти выражения с результатами (4.40) и (4.41), сразу получим

$$
F_{1}=-\frac{1}{24} \ln M_{1}-\frac{1}{24} \ln J_{1}-\frac{1}{6} \ln d,
$$

что совпадает с выражением в работе [60].

В обшем случае все не так просто. Базисные векторы, вообше говоря, не представляются в виде полных производных, поскольку коэффициенты $A$ и $D$ теперь зависят от потенциала более сложным образом. Тем не менее, если переписать базисные векторы в более удобном виде, можно доказать, что $W_{g}(p)$ есть полная производная. Переписанные таким образом векторы $\chi^{(n)}(p)$ имеют вид

$$
\begin{aligned}
\chi^{(n)}(p)= & \frac{1}{M_{1}}\left\{-\frac{1}{2 n-1} \sum_{i=1}^{n-1}(-1)^{n-i-1}\left\{\Phi_{x}^{(i)}-M_{i} \frac{d y}{d V(p)}\right\}\left(\frac{1}{x-y}\right)^{n-i}-\right. \\
& \left.-\frac{2}{2 n-1} \frac{d M_{n-1}}{d V(p)}-\sum_{k=2}^{n-1} \chi^{(k)} M_{n-k+1}\right\}, \quad n \geqslant 2,
\end{aligned}
$$

где $\Phi_{x}^{(n)}$, в свою очередь, должно быть представлено как

$$
\begin{aligned}
\Phi_{x}^{(n)}= & -\frac{1}{2 n-1} \sum_{i=1}^{n-1}(-1)^{n-i-1}\left\{\Phi_{x}^{(i)}-M_{i} \frac{d y}{d V(p)}\right\}\left(\frac{1}{x-y}\right)^{n-i}+ \\
& +M_{n} \frac{d x}{d V(p)}-\frac{2}{2 n-1} \frac{d M_{n-1}}{d V(p)}, \quad n \geqslant 2 \\
\Phi_{x}^{(1)}= & M_{1} \frac{d x}{d V(p)} .
\end{aligned}
$$

Базисный вектор $\chi^{(1)}(p)$ по-прежнему имеет вид (4.44). Величины $\Psi^{(n)}$ переписываются аналогично $\chi^{(n)}$. Они получаются при заменах $J \leftrightarrow M$ и $x \leftrightarrow y$ в вышеприведенных формулах. 
В результате таких переписываний можно найти

$$
\begin{aligned}
F_{2}= & -\frac{181}{480 J_{1}^{2} d^{4}}-\frac{181}{480 M_{1}^{2} d^{4}}+\frac{181 J_{2}}{480 J_{1}^{3} d^{3}}-\frac{181 M_{2}}{480 M_{1}^{3} d^{3}}+ \\
& +\frac{3 J_{2}}{64 J_{1}^{2} M_{1} d^{3}}-\frac{3 M_{2}}{64 J_{1} M_{1}^{2} d^{3}}-\frac{11 J_{2}^{2}}{40 J_{1}{ }^{4} d^{2}}-\frac{11 M_{2}^{2}}{40 M_{1}^{4} d^{2}}+ \\
& +\frac{43 M_{3}}{192 M_{1}^{3} d^{2}}+\frac{43 J_{3}}{192 J_{1}^{3} d^{2}}+\frac{J_{2} M_{2}}{64 J_{1}^{2} M_{1}^{2} d^{2}}-\frac{5}{16 J_{1} M_{1} d^{4}}+ \\
& +\frac{21 J_{2}^{3}}{160 J_{1}^{5} d}-\frac{29 J_{2} J_{3}}{128 J_{1}^{4} d}+\frac{35 J_{4}}{384 J_{1}{ }^{3} d}-\frac{21 M_{2}{ }^{3}}{160 M_{1}^{5} d}+ \\
& +\frac{29 M_{2} M_{3}}{128 M_{1}^{4} d}-\frac{35 M_{4}}{384 M_{1}^{3} d} .
\end{aligned}
$$

Обобщение техники моментов на случай суперсимметричной модели собственных значений $[85]^{7)}$ было найдено в работе [86], где вычисления также ограничивались родом два.

4.3. Двойной скейлинговый предел. Легко увидеть, какие члены в явных выражениях для $F_{g}$ и $W_{g}(p)$, полученных в предыдушем пункте, ответственны за ДСП. Отметим, что нахождение величин $F_{g}$ и $W_{g}(p)$ вне ДСП, как было показано, достаточно трудоемкая процедура уже начиная с рода два. Поэтому в работе [53] был разработан алгоритм, позволяющий получать результаты прямо в ДСП, что позволило найти корреляторы и статистическую сумму в ДСП до рода четыре включительно.

4.3.1. Критические точки. Обратимся к случаю несимметричного потенциала и рассмотрим $m$-ю критическую точку, предполагая, что дополнительные нули собираются в точке $x$. Чтобы получить ДСП корреляторов, положим отношение между некоторой выбранной константой связи и первой нетривиальной константой равным критическому значению. С делаем масштабное преобразование переменных $p$ и $x$ следующим образом, оставляя при этом переменную $y$ конечной:

$$
\begin{aligned}
& p=x_{c}+a \pi, \\
& x=x_{c}-a \Lambda^{\frac{1}{m}} .
\end{aligned}
$$

При этом моменты $J_{k}$ не масштабируются, а поведение моментов $M_{k}$ имеет вид

$$
M_{k} \sim a^{m-k}, \quad k \in[1, m-1] .
$$

\footnotetext{
7) Вообще говоря, суперсимметричные обобщения матричных моделей затруднены прежде всего потому, что величины, обычно исследуемые в этой технике, - это статистические суммы, которые для ненарушенной суперсимметрии должны быть тривиальными. Чтобы обойти эту трудность предлагались различные техники. Одной из них (и, пожалуй, самой первой по времени возникновения) и была суперсимметричная модель собственных значений типа $m, n$, т.е. модель с потенциалом и (супер)определителем Вандермонда, зависящими от $m$ коммутирующих и $n$ антикоммутирующих переменных ("собственных значений”, по-видимому, несуществующей матрицы).
} 
Поскольку $F_{g} \sim a^{(2-2 g)(m+1 / 2)}$, получим, что в ДСП для $F_{g}$ остаются лишш те члены, для которых

$$
\begin{gathered}
N_{M}=2-2 g, \\
\sum_{j=1}^{s}\left(\alpha_{j}-1\right)=3 g-3 .
\end{gathered}
$$

Сравнивая (4.56) с (4.22), получим, что вся зависимость от моментов $J_{k}$ исчезает при взятии ДСП в соответствии с формулами (4.53) и (4.54). Далее, сравнивая (4.57) с (4.23), найдем, что для всех оставшихся членов $\gamma=g-1$.

Поскольку базисные векторы ведут себя как $\chi^{(n)} \sim a^{-m-n+1 / 2}$ и $\Psi^{(n)} \sim a^{-1 / 2}$, а петлевое среднее

$$
W_{g}(p) \sim a^{(1-2 g)\left(m+\frac{1}{2}\right)-1}
$$

при масштабных преобразованиях ${ }^{8)}$, то можно получить условия однородности, которым должны удовлетворять коэффициенты $A$ и $D$ :

$$
\begin{array}{ll}
A_{g}^{(n)}: & N_{M} \geqslant 2-2 g, \quad \sum_{j=1}^{s}\left(\alpha_{j}-1\right) \leqslant 3 g-n-1, \\
D_{g}^{(n)}: & N_{M} \geqslant 1-2 g, \quad \sum_{j=1}^{s}\left(\alpha_{j}-1\right) \leqslant 3 g-1 .
\end{array}
$$

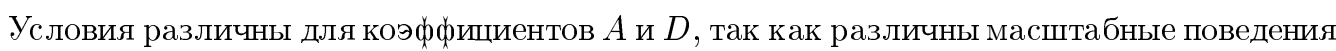
базисных векторов. В ДСП важны лишы те члены, для которых $s \leqslant m$ и оба неравенства в (4.59) или (4.60) обрашаются в равенства. При этом все $D$-члены дают нулевой вклад. Более того, сравнивая (4.59) с (4.33), получим, что и все $A$-члены с нетривиальной зависимостью от $J_{k}$ не дают вклада в ДСП. Поэтому в ДСП все величины выражаются только через моменты $M_{k}$ и $d$, и во всех этих членах $\gamma=g-1$.

Найдем алгоритм, который сразу бы выделял члены, даюшие вклад в непрерывном пределе. Легко видеть, что члены, даюшие вклад в ДСП петлевого коррелятора $W_{g}(p)$, возникают из членов, отличных от нуля в ДСП в правой части петлевого уравнения. Допустим, что известны масштабно-зависимые формулы для $A_{G}^{(n)}$ и $D_{G}^{(n)}$ при $G=1, \ldots, g$ и необходимо вычислить ДСП правой части петлевого уравнения для $W_{g+1}$. Заметим, что все члены, входяшие в базисные векторы $\chi^{(n)}(p)$ и $\Psi^{(n)}(p)$, имеют одно и то же масштабное поведение, и потому ни один из этих членов не может быть опущен. Однако в этом пределе можно всюду заменить $(p-y)$ на $\left(x_{c}-y\right)=d_{c}$. Из предыдущего анализа известно [87], что в $m$-мультикритической модели $W_{g}(p, p)$ имеет масштабное поведение

$$
W_{g}(\pi, \pi) \sim a^{-2 g\left(m+\frac{1}{2}\right)-2} .
$$

\footnotetext{
${ }^{8)}$ Кроме члена $W_{0}(p)$, который содержит также и нескейлинговую часть.
} 
Используя асимптотики (4.58), легко показать, что все произведения в сумме в формуле (4.8) имеют одно и то же масштабное поведение. Поэтому можно сразу начинать анализ с выражений для $W_{G}, G=1, \ldots, g$, получаемых в ДСП.

Если вычислять $W_{g}(p, p)$, применяя оператор вставки петель по предписанию в (4.42) и действуя им на $W_{g}(p)$, то лишние слагаемые возникают даже в случае, когда используется выражение для $W_{g}(p)$, отвечающее ДСП. Сравнивая (4.58) и (4.61), увидим, что нужные слагаемые порождаются только теми операторами из $d / d V(p)$, которые понижают степень $a$ на $m+3 / 2$. Поэтому все прочие операторы должны быть опущены, и в ДСП остается только следующая часть оператора вставки петель:

$$
\frac{d}{d V(p)}=\sum_{n} \frac{d M_{n}}{d V(p)} \frac{\partial}{\partial M_{n}}+\frac{d x}{d V(p)} \frac{\partial}{\partial x} \quad(\text { ДСП })
$$

где

$$
\begin{aligned}
& \frac{d M_{n}}{d V(p)}=-\left(n+\frac{1}{2}\right)\left\{\Phi_{x}^{(n+1)}(p)-\frac{M_{n+1}}{M_{1}} \Phi_{x}^{(1)}(p)\right\} \quad(\text { ДСП }) \\
& \frac{d x}{d V(p)}=\frac{1}{M_{1}} \Phi_{x}^{1}(p) \quad(\text { ДСП })
\end{aligned}
$$

и

$$
\Phi_{x}^{(n)}(p)=(p-x)^{-n}\left\{d_{c}(p-x)\right\}^{-\frac{1}{2}} \quad(\text { ДСП). }
$$

В дальнейшем алгоритм в точности совпадает с алгоритмом вывода пертурбативного решения модели Концевича в терминах моментов [38]. Найдем теперь $F_{g}$ в ДСП. Начнем с выражения для $W_{g}(p)$ в ДСП, и стратегия будет по-прежнему состоять в том, чтобы представить базисные векторы в виде, позволяющем написать $W_{g}(p)$ как полную производную. Такое представление для $\chi^{(n)}$ описьвается формулой (4.63) вместо (4.43) и имеет вид

$$
\chi^{(n)}(p)=\frac{1}{M_{1}}\left\{-\frac{2}{2 n-1} \frac{d M_{n-1}}{d V(p)}-\sum_{k=2}^{n-1} \chi^{(k)}(p) M_{n-k+1}\right\}, \quad n \geqslant 2 .
$$

Выражение для $\chi^{(1)}(p)$ не требуется, поскольку сумма в $(4.66)$ начинается с $k=2$ и известно, что в ДСП $A_{g}^{(1)}=0$. Также не нужно знать выражения для $\Phi_{x}(p)$. Правильная замена в $\Psi^{(n)}(p)$ задается (4.66), где $M$ заменено на $J$.

В случае несимметричного потенциала, в котором критическое поведение связано с концевой точкой $x$, получим для рода один

$$
F_{1}^{(\mathrm{NS})}=-\frac{1}{24} \ln M_{1} \quad \text { (ДСП). }
$$

Общая структура $F_{g}^{(\mathrm{NS})}$, возникающая при итеративном решении петлевого уравнения, имеет вид

$$
F_{g}^{(\mathrm{NS})}=\sum_{\alpha_{j}>1}\left\langle\alpha_{1} \ldots \alpha_{s} \mid \alpha, \gamma\right\rangle_{g}^{\mathrm{herm}} \frac{M_{\alpha_{1}} \ldots M_{\alpha_{s}}}{M_{1}^{\alpha} d^{\gamma}} \quad \text { (ДСП), } \quad g \geqslant 1,
$$


где рациональные числа ("индексы пересечений") в точности равны соответствующим числам из формул (4.18) и (2.17). Величины $\alpha_{j}$ удовлетворяют условию $(4.57), \mathrm{a} \gamma=$ $g-1$, причем

$$
\alpha=2 g-2+s,
$$

как следует из (4.56).

Рассмотрим случай $g=2$, из которого будет ясно, какие структуры содержатся в (4.68). Для $g=2$ имеем $\alpha_{j} \in[2,3 g-2]=[2,4]$, и из соотношения (4.57) следует, что

$$
\sum_{j=1}^{s}\left(\alpha_{j}-1\right)=3 g-3=3, \quad g=2 ;
$$

таким образом $s \in[1,3 g-3]=[1,3]$. Условие (4.70) накладывает ограничения на допустимые значения $s=3$ и $\alpha_{1}=\alpha_{2}=\alpha_{3}=2, s=2$ и $\alpha_{1}, \alpha_{2}=2,3$ и $s=1$ и $\alpha_{1}=4$. Степени момента $M_{1}$ в знаменателе равны $\alpha=2 g-2+s=2+s$. В итоге получим

$$
F_{2}^{(\mathrm{NS})}=-\frac{21 M_{2}{ }^{3}}{160 d_{c} M_{1}{ }^{5}}+\frac{29 M_{2} M_{3}}{128 d_{c} M_{1}{ }^{4}}-\frac{35 M_{4}}{384 d_{c} M_{1}{ }^{3}} .
$$

Заметим, что в этой формуле ненулевыми оказались все принципиально возможные значения $s$ и $\alpha_{i}$. Простое исследование оператора вставки петли и базисных векторов показывает, что это же условие верно для всех родов (это же следует из математических соображений - согласно Концевичу индексы пересечений строго положительны). Ответы для $g=3$ (где $s \leqslant 6$ ) и $g=4$ (где $s \leqslant 9$ ) оказываются уже достаточно громоздкими [53]. Для симметричного потенциала возникает сумма двух совпадаюших членов, поэтому

$$
F_{g}^{(\mathrm{S})}=2 F_{g}^{(\mathrm{NS})} \quad(\text { ДСП }),
$$

т.е. получается известное свойство ДСП в 1ММ [88]. (На самом деле, в ДСП петлевое уравнение для симметричного потенциала распадается на два независимых уравнения. Заметим, что результаты раздела 6 показывают, что в параметризации через времена ДПМ статистическая сумма представляется через два интеграла ММК, связанных оператором канонического преобразования времен, которьй становится единичным оператором в ДСП.) Формула (4.72) объясняет, почему статистическая сумма двумерной квантовой гравитации определялась как квадратньй корень от выражения для статистической суммы модели (2.1) в ДСП для симметричного потенциала. В несимметричном случае квадратного корня не появляется и правильная статистическая сумма непрерывной модели дается простым ДСП выражения (2.1).

4.3.2. Предельная процедура. В формуле (4.68) для ДСП свободной энергии содержится вся информашия о возможных мультикритических точках 1ММ. Из теоремы 3 следует, что в этом пределе должна возникать ММК.

Покажем теперь, что ММК может быть получена из 1MМ с помошью определенной предельной процедурьи, которая имеет вид, отличный (на первый взгляд) от ДСП. Однако в п. 4.3.3 будет показано, что эта предельная процедура дает ответ, идентичный ответу в ДСП. 
Начнем с ряда тождеств. 1ММ эквивалентна ММКП (2.37) (см. формулу (3.36)). Сделаем замену переменных

$$
\tilde{\Lambda} \rightarrow e^{\varepsilon \Lambda}, \quad \alpha \rightarrow \frac{1}{\varepsilon^{3}},
$$

где новую матрицу $\Lambda$ надо отождествить с матрицей внешнего поля $\mathrm{MMK}(2.17)$, а $\varepsilon-$ параметр разложения, который в дальнейшем будет стремиться к нулю (в разделе 2 этот параметр играет роль шага дискретизации пространств модулей).

Теперь легко проверить, что в пределе $\varepsilon \rightarrow 0$ модель $(2.17)$ получается из $(2.37)$ в результате масштабного преобразования $X \rightarrow \varepsilon X$. Тем самым построен гладкий переход между интегралами $Z_{H}\left(\left\{\xi_{k}\right\},-\alpha N\right)$ и $Z_{\mathrm{K}}(\Lambda, N)$. Заметим, что нет необходимости брать предел $N \rightarrow \infty$ в интегралах, участвующих в этом переходе, но размер $M=-\alpha N$ исходных эрмитовых матриц стремится к бесконечности при $\varepsilon \rightarrow 0$.

Полезно проиллюстрировать это доказательство, вычисляя предел $\varepsilon \rightarrow 0$ в явных формулах, описывающих 1MМ. Основные уравнения (4.10), которые в исходной 1ММ определяют концевые точки $x$ и $y$ распределения собственных значений через константы связи $\xi_{i}$, имеют свои полные аналоги в терминах собственных значений $\eta_{j}$ матрищы $\eta$, если переписать $1 \mathrm{MM}$ в терминах OMK (3.18). Соответствующие уравнения получаются при подстановке в (4.10) следуюшего соотношения между собственными значениями $\eta_{j}$ и потенциалом $V$ эрмитовой матричной модели:

$$
V^{\prime}(\omega)=\frac{1}{N} \sum_{j=1}^{N} \frac{1}{\eta_{j}-\omega}-\omega .
$$

Это соотношение прямо следует из преобразования Конщевича-Мивы (3.22). Применяя (4.74), получим для уравнений (4.10)

$$
\begin{gathered}
\frac{1}{N} \sum_{i=1}^{n} \frac{1}{\sqrt{\left(\eta_{i}-x\right)\left(\eta_{i}-y\right)}}-\frac{x+y}{2}=0, \\
\frac{1}{N} \sum_{i=1}^{n} \frac{\eta_{i}-\frac{x+y}{2}}{\sqrt{\left(\eta_{i}-x\right)\left(\eta_{i}-y\right)}}-\frac{(x-y)^{2}}{8}=-2 \alpha .
\end{gathered}
$$

Заметим, что нормировочный фактор 2 в (4.10) изменен здесь на $-2 \alpha$, так как размер матрицы теперь равен $M=-\alpha N$.

Применяя предельный переход (4.73) к уравнениям (4.75) и (4.76), получим

$$
\eta \rightarrow \sqrt{\alpha}\left(e^{\varepsilon \Lambda}+e^{-\varepsilon \Lambda}\right)=\frac{\sqrt{2}}{\varepsilon^{\frac{3}{2}}}+\sqrt{\frac{\varepsilon}{2}} \Lambda^{2}+O\left(\varepsilon^{\frac{5}{2}}\right) .
$$

Чтобы решить уравнения $(4.75),(4.76)$ относительно $x$ и $y$, выберем

$$
y=-\frac{\sqrt{2}}{\varepsilon^{\frac{3}{2}}}, \quad x=\frac{\sqrt{2}}{\varepsilon^{\frac{3}{2}}}+\sqrt{2} \varepsilon^{\frac{1}{2}} u_{0}+\cdots .
$$


Из (4.75) следует уравнение

$$
\frac{1}{N} \sum_{j=1}^{N} \frac{1}{\sqrt{\lambda_{j}^{2}-2 u_{0}}}=u_{0}
$$

которое возникает при члене, пропорциональном $\sqrt{\varepsilon}$. В результате подстановки указанных величин в (4.76) получим, что ведуший вклад порядка $1 / \varepsilon^{3}$, содержащийся в членах $2 \alpha$ и $(x-y)^{2} / 8$, сокрашается. Для этого необходимо, чтобы $\xi>0$. Члены следуюшего порядка, пропорциональные $1 / \varepsilon$, комбинируются в уравнение (4.79), которое и есть не что иное, как условие стационарности [47], [89], возникающее в ММК.

ДСП в модели с симметричным потенциалом, где все нечетные времена $\xi_{n}$ равны нулю, имеет свою специфику - в этом случае надо выбирать преобразование исходной внешней матрицы в блочно-диагональном виде:

$$
\Lambda \rightarrow \operatorname{diag}\left(e^{\varepsilon \lambda_{1}}, \ldots, e^{\varepsilon \lambda_{N / 2}}, e^{-\varepsilon \lambda_{1}}, \ldots, e^{-\varepsilon \lambda_{N / 2}}\right) .
$$

Тогда в пределе $\varepsilon \rightarrow 0$ получаются два независимых интеграла ММК по эрмитовым матрицам половинной размерности $N / 2 \times N / 2$ [53], взятых с одним и тем жее внешним матричным полем $\tilde{\Lambda}=\operatorname{diag}\left(\lambda_{1}, \ldots, \lambda_{N / 2}\right)$.

4.3.3. Моменты ММК. Эквивалентность ММК и ДСП 1ММ для рода ноль была показана в работах [47], [89], где сравнивались явные выражения. Для того чтобы сравнить следуюшие порядки в разложении по родам, рассмотрим выражение для вклада рода $g$ в свободную энергию $\mathrm{MMK} F_{g}^{\mathrm{K}}$, которое было доказано в работе Итциксона и Зубера [38]:

$$
F_{g}^{\mathrm{K}}=\sum_{2 \leqslant k \leqslant 3 g-2} \sum_{(k-1) l_{k}=3 g-3}\left\langle\tau_{2}^{l_{2}} \tau_{3}^{l_{3}} \ldots \tau_{3 g-2}^{l_{3 g-2}}\right\rangle_{g}^{\mathrm{K}} \frac{1}{\left(1-I_{1}\right)^{2(g-1)+\sum l_{p}}} \frac{I_{2}^{l_{2}}}{l_{2} !} \frac{I_{3}^{l_{3}}}{l_{3} !} \frac{I_{3 g-2}^{l_{3 g-2}}}{l_{3 g-2} !},
$$

где $g \geqslant 2$, моменты $I_{k}$ даются формулой 9$)$

$$
I_{k}(M)=\frac{1}{n} \sum_{j=1}^{n} \frac{1}{\left(m_{j}^{2}-2 u_{0}\right)^{k+\frac{1}{2}}}, \quad k \geqslant 0,
$$

а параметр $u_{0}(M)$ определяется уравнением $u_{0}=I_{0}\left(u_{0}, M\right)($ см. (4.79)). Это уравнение называется также струнньм уравнением. Сравнивая коэффициенты $\langle\cdot\rangle_{g}^{\mathrm{K}}$, вычисленные в работе [38], с $\langle\cdot\rangle_{g}^{\text {herm }}$, найденными в ДСП, увидим, что они совпадают. Покажем, что это происходит для всех родов.

Возврашаясь к ответу (4.80), заметим, что сами переменные $I_{k}$ можно рассматривать как новые времена МMK. Поскольку сама статистическая сумма MMK есть $\tau$-функция Кд $\Phi$, то она удовлетворяет всей иерархии уравнений Кд $\Phi$, из которой здесь понадобится

\footnotetext{
9) Определение $(4.81)$ отличается на множитель $-(2 k-1)$ !! от определения, данного для $I_{k}$ в работе [38].
} 
лишь первое. Если ввести величину $u_{g} \equiv d^{2} \ln F_{g} / d t_{0}^{2}$, то первое уравнение иерархии КдФ в разложении по родам примет вид

$$
\frac{\partial u_{g}}{\partial t_{1}}=\frac{\partial}{\partial t_{0}}\left(\frac{1}{12} \frac{\partial^{2} u_{g-1}}{\partial t_{0}^{2}}+\frac{1}{2} \sum_{g_{1}+g_{2}=g} u_{g_{1}} u_{g_{2}}\right) .
$$

Кроме того, легко получить формулы дифференцирования моментов $I_{k}$ :

$$
\begin{gathered}
\frac{\partial u_{0}}{\partial t_{0}}=\frac{1}{1-I_{1}}, \quad \frac{\partial u_{0}}{\partial t_{1}}=\frac{u_{0}}{1-I_{1}}, \\
\frac{\partial I_{p}}{\partial t_{0}}=\frac{I_{p+1}}{1-I_{1}}, \quad p \geqslant 1, \\
\left(\frac{\partial}{\partial t_{1}}-u_{0} \frac{\partial}{\partial t_{0}}\right) I_{p}=\delta_{p, 1}, \quad p \geqslant 1 .
\end{gathered}
$$

Если сделать еще одну замену и ввести времена

$$
\xi_{k}=\frac{I_{k}}{\left(1-I_{1}\right)^{k}}, \quad k \geqslant 2
$$

то из формулы (4.80) будет следовать, что

$$
F_{g}=f_{g}\left(\left\{\xi_{k}\right\}\right)\left(1-I_{1}\right)^{g-1} .
$$

Тогда, вводя дифференциальные операторы первого порядка

$$
S_{0} \equiv \sum_{s=2}^{\infty}\left(\xi_{s+1}+s \xi_{2} \xi_{s}\right) \frac{\partial}{\partial \xi_{s}}-\xi_{2} w \frac{\partial}{\partial w}, \quad S_{1} \equiv \sum_{s=2}^{\infty} s \xi_{s} \frac{\partial}{\partial \xi_{s}}-w \frac{\partial}{\partial w}-1
$$

и производящую функцию

$$
f(w) \equiv S_{0}^{2} \sum_{g=1}^{\infty} w^{g-1} f_{g}
$$

получим дифференциальное уравнение

$$
S_{1} f(w)=\frac{w}{12} S_{0}^{3} f(w)+w f(w) S_{0} f(w)
$$

которое полностью определяет все индексы пересечений для всех родов, как только известен ответ для рода один $\left(f_{1}=\left(\xi_{3}+\xi_{2}^{2}\right) / 24\right)$.

Например, струнное уравнение (4.79) в обозначениях [38] можно переписать в виде

$$
u_{0}=I_{0}\left(\{t .\}, u_{0}\right) \equiv t_{0}+\sum_{k=1}^{\infty} \frac{t_{k}}{k !} u_{0}^{k} \equiv t_{0}+J\left(u_{0}\right) .
$$


Разрешая его итеративно по степеням $J\left(t_{0}\right)$ (т.е. считая $t_{0}$ конечным и полагая остальные времена малыми параметрами разложения), получим

$$
\begin{aligned}
u_{0} & =t_{0}+\sum_{n=1}^{\infty} \frac{1}{n !}\left(\frac{d}{d t_{0}}\right)^{n-1} J\left(t_{0}\right)= \\
& =t_{0}+\sum_{1 \leqslant \sum_{1}^{\infty} r_{k}<\infty} \frac{\left(\sum_{k=1}^{\infty} k r_{k}\right) !}{\prod_{k=1}^{\infty}(k !)^{r_{k}}} \frac{t_{0}^{\sum_{k=1}^{\infty}(k-1) r_{k}+1}}{\left(\sum_{k=1}^{\infty}(k-1) r_{k}+1\right) !} \prod_{k=1}^{\infty} \frac{t_{k}^{r_{k}}}{r_{k} !},
\end{aligned}
$$

что дает после интегрирования дважды по $t_{0}$

$$
F_{0}=\sum_{\substack{r_{k}>0 \\ \sum_{k=0}^{\infty}(k-1) r_{k}=-3}} \frac{\left(\sum_{k=1}^{\infty} k r_{k}\right) !}{\prod_{k=1}^{\infty}(k !)^{r_{k}}} \prod_{k \geqslant 0}^{\infty} \frac{t_{k}^{r_{k}}}{r_{k} !},
$$

откуда

$$
\left\langle\tau_{d_{1}} \ldots \tau_{d_{n}}\right\rangle_{0}=\frac{\left(\sum_{i} d_{i}\right) !}{\prod_{i=1}^{n} d_{i} !} \quad \text { при } \quad \sum_{i=1}^{n} d_{i}=n-3
$$

что совпадает с результатом из работы [78], полученным из когомологических соображений.

Если ограничиться, например, старшим классом когомологий (с одной выколотой точкой), то в выражении (4.85) от оператора $S_{0}$ останется лишь часть, пропорциональная $\xi_{s+1} \partial / \partial_{\xi_{s}}$, второй член в правой части (4.87) также не будет давать вклада, и можно легко получить рекуррентные соотношения, из которых следует результат [21]

$$
\left\langle\tau_{3 g-2}\right\rangle_{g}=\frac{1}{24^{g} g !} .
$$

Для любого значения $\varepsilon$ в (4.73) имеет место разложение (4.18) свободной энергии $F_{g}$ эрмитовой матричной модели в терминах моментов $M_{k}$ и $J_{k}$. Покажем, что члены, остаюшиеся в пределе $\varepsilon \rightarrow 0,-$ это в точности те самые члены, которые не обрашаются в нуль в ДСП (4.68), и что $M_{k}$ переходят непосредственно в моменты $I_{k}$ модели Концевича, определенные (4.81) и (4.79).

Обсудим масштабное поведение моментов $M_{k}$ и $J_{k}$. Подставляя (4.74) в выражения (4.13) и (4.14), получим

$$
\begin{aligned}
M_{k} & =\frac{1}{N} \sum_{j} \frac{1}{\left(\eta_{j}-x\right)^{k+\frac{1}{2}}\left(\eta_{j}-y\right)^{\frac{1}{2}}}-\delta_{k 1}, \quad k \geqslant 1, \\
J_{k} & =\frac{1}{N} \sum_{j} \frac{1}{\left(\eta_{j}-x\right)^{\frac{1}{2}}\left(\eta_{j}-y\right)^{k+\frac{1}{2}}}-\delta_{k 1}, \quad k \geqslant 1 .
\end{aligned}
$$


Из этого представления и соотношений $(4.77),(4.78)$ для моментов $M_{k}, J_{k}$ и параметра $d$ легко вывести следуюшие формулы для масштабного поведения:

$$
\begin{aligned}
J_{k} & \rightarrow-2^{-\left(\frac{3 k}{2}+1\right)} \varepsilon^{\frac{3 k+1}{2}} I_{0}+\delta_{k 1}, \\
M_{k} & \rightarrow-2^{\frac{k-1}{2}} \varepsilon^{-\frac{k-1}{2}}\left(I_{k}-\delta_{k 1}\right), \\
d & \rightarrow 2^{\frac{3}{2}} \varepsilon^{-\frac{3}{2}}
\end{aligned}
$$

где $I_{k}$ - в точности моменты ММК, определенные формулами (4.81), (4.79).

Обратимся к обшему выражению (4.18) для $F_{g}$. В нем степень параметра $\varepsilon$, стояшего при члене

$$
\frac{M_{\alpha_{1}} \ldots M_{\alpha_{s}} J_{\beta_{1}} \ldots J_{\beta_{l}}}{d^{\gamma} M_{1}^{\alpha} J_{1}^{\beta}}
$$

равна

$$
[\varepsilon]=\sum_{i=1}^{l} \frac{3 \beta_{i}+1}{2}-\sum_{j=1}^{s} \frac{\alpha_{j}-1}{2}+\frac{3}{2} \gamma .
$$

Из формул (4.23) и (4.25) следует, что $[\varepsilon] \geqslant 0$. Равенство $[\varepsilon]=0$ возможно тогда и только тогда, когда $l=0, \gamma=g-1$ и

$$
\sum_{j=1}^{s}\left(\alpha_{j}-1\right)=3 g-3
$$

Тем самым выживают в точности те же члены, что и в общей процедуре ДСП (4.68). Исследование оператора вставки петли (4.42) позволяет заключить, что такие члены всегда входят с параметром $\beta=0$, а следовательно, $N_{M}=2-2 g$. Таким образом, если исходить из $1 \mathrm{MM} \mathrm{общего} \mathrm{вида} \mathrm{и} \mathrm{вычислить} \mathrm{предел} \varepsilon \rightarrow 0$, то можно воспроизвести формулы (4.80), (4.81) и (4.79), причем коэффициенты, с которыми входят моменты $I_{k}$, совпадают с соответствуюшими коэффициентами из 1ММ. Таким образом, получим $\langle\cdot\rangle_{g}^{\text {herm }}=\langle\cdot\rangle_{g}^{\mathrm{K}}$, поскольку только что было явно показано, что статистическая сумма 1 ММ совпадает со статистической суммой ММК в пределе $\varepsilon \rightarrow 0, \alpha=\varepsilon^{-3}$.

Струнная восприимчивость $\gamma_{\mathrm{str}}$ связана со второй полной производной от $F_{0}$ по параметру $\alpha$. Оказывается, что ввиду условия стационарности вторая полная производная совпадает с частной производной:

$$
\chi_{\mathrm{KP}}=\frac{1}{N^{2}} \frac{d^{2}}{d \alpha^{2}} F_{0}=4 \ln d .
$$

Последнее замечание касается того, как изменится петлевое уравнение (4.8) в модели собственных значений (3.6). Из условий (3.7) получим

$$
\oint_{C} \frac{d \omega}{2 \pi i} \frac{V^{\prime}(\omega)}{p-\omega} W(\omega)=\frac{\kappa}{2} W^{2}(p)+\frac{\kappa}{2} \frac{1}{M^{2}} W(p, p)+\frac{\frac{\kappa}{2}-1}{M} W^{\prime}(p),
$$


где главное отличие от формулы (4.8) (помимо тривиальных масштабных преобразований) заключается в последнем члене в правой части уравнения. В случае, когда $\kappa \sim$ $O(1)$ относительно параметра $M$, этот член несушествен в пределе $M \rightarrow \infty$, и $W_{0}(p)$ имеет вид (4.9), а поправки могут быть вычислены из (4.102). Более интересным представляется вопрос о вычислении (4.102) в случае, когда член с производной становится того же порядка по $1 / M$, что и остальные члены бездисперсионного петлевого уравнения (такой предел может описывать КТП с центральными зарядами, отличными от $c=1)$. Этот вопрос требует дальнейших исследований.

\section{5. ОБОБЩЕННЫЕ МОДЕЛИ КОНЦЕВИЧА С НЕПОЛИНОМИАЛЬНЫМИ ПОТЕНЦИАЛАМИ}

5.1. Матричная модель суперструны IIB. В данном пункте обсуждаются матричные модели, описываюшие непертурбативные эффекты (эффективное действие) суперструнных теорий. Помимо работы [23] и развитых впоследствии подходов теории возмушений, используюших матричные модели [90]-[93], альтернативный (и более близкий к собственно матрично-модельному описанию) подход был предложен в работе [24], где матричная модель непосредственно отождествлялась с суперструной типа IIB. Действие этой модели (модели Ишибаши-Каваи-Китазавы-Тсучии (ИККТ)) получается из действия десятимерной $U(N)$-супертеории Янга-Миллса при редукции в точку (нулевое измерение, соответствующее приближению квантовой механики). Струнное действие при этом получается из матричной модели, если коммутаторы заменить на скобки Пуассона, а матричные следы - на интегралы по мировой поверхности струны. Эта замена оправдана при бесконечных $N$, если интерпретировать предел больших $N$ как квазиклассическое приближение. При этом возникает действие Шилда IIB суперструны Грина-Шварца [94], [95] с фиксированной $\kappa$-симметрией:

$$
S_{\text {Schild }}=\int d^{2} \sigma\left(\frac{\alpha}{4 \sqrt{g}}\left\{X^{\mu}, X^{\nu}\right\}_{\mathrm{PB}}^{2}-\frac{i}{2} \bar{\psi} \Gamma^{\mu}\left\{X_{\mu}, \psi\right\}_{\mathrm{PB}}+\beta \sqrt{g}\right),
$$

где $\{\cdot, \cdot\}_{\text {РВ }}$ - стандартные скобки Пуассона.

Действие Шилда (5.1) становится эквивалентным действию Намбу-Гото, если исключить вспомогательное поле $\sqrt{g}$, используя классические уравнения движения. На квантовом уровне это соответствует усреднению по полю $\sqrt{g}$ в функциональном интеграле. Такой механизм получения эффективного действия должен воспроизводить поляковскую струну при подходящем выборе меры интегрирования и сокращении конформной аномалии [96].

В подходе [24] интегрированию по $\sqrt{g}$ отвечает суммирование по размеру матриц $N$, который, таким образом, становится динамической переменной. (Заметим, что это созвучно преобразованию (3.28), в котором размер матриц 1ММ переходит в (динамическую) константу при логарифмическом члене ММКП.) В работе [63] (см. также обзор [97]) была предложена модификация модели ИККТ, в которой дополнительная матричная переменная $Y$ входит таким же образом, как поле $\sqrt{g}$ входит в струнное действие:

$$
S_{\mathrm{NBI}}=-\frac{\alpha}{4} \operatorname{tr} Y^{-1}\left[A_{\mu}, A_{\nu}\right]^{2}+\beta \operatorname{tr} Y-\frac{1}{2} \operatorname{tr} \bar{\psi} \Gamma^{\mu}\left[A_{\mu}, \psi\right]
$$


(NBI - это аббревиатура неабелева действия Борна-Инфельда). В этой модели нет суммирования по $N$, и в пределе больших $N$ (квазиклассическое приближение) получается струнное действие (5.1).

Как уже подчеркивалось, основная идея любого подхода матричных моделей в теории струн - это принять матричный интеграл в пределе $N \rightarrow \infty$ в качестве непертурбативного определения статистической суммы струны. Здесь главная проблема состоит в выборе меры интегрирования, которая должна сохранять все симметрии исходной струнной теории. В работе [63] была предложена следующая статистическая сумма:

$$
Z_{\mathrm{NBI}}=\int d A_{\mu} d \bar{\psi} d \psi d Y(\operatorname{det} Y)^{-\gamma} e^{-S_{\mathrm{NBI}}}
$$

Интегрирование по $Y$ производится по положительно определенным эрмитовым матрицам. Эффективное действие и мера для поля Янга-Миллса $A_{\mu}$ и его суперпартнеров получается в результате этого интегрирования:

$$
J(G) \exp \left\{-S_{\text {eff }}(G)+\frac{1}{2} \operatorname{tr} \bar{\psi} \Gamma^{\mu}\left[A_{\mu}, \psi\right]\right\}=\int d Y(\operatorname{det} Y)^{-\gamma} e^{-S_{\mathrm{NBI}}}
$$

где

$$
G=-\left[A_{\mu}, A_{\nu}\right]^{2}
$$

- эрмитова положительно-определенная матрица. При больших $N$, когда коммутатор заменяется на скобку Пуассона, поле $G$ становится детерминантом индуцированной метрики на мировой поверхности струны:

$$
G \rightarrow\left\{X_{\mu}, X_{\nu}\right\}_{\mathrm{PB}}^{2}=\operatorname{det}_{a b} \partial_{a} X_{\mu} \partial_{b} X^{\mu}
$$

Заметим, что поле $Y$ не взаимодействует с фермионами и соответствующий член в действии не меняется. Также следует отметить, что разделение результата интегрирования по $Y$ на эффективное действие и меру интегрирования содержит определенный произвол. Как правило, все степенные члены в показателе экспоненты интерпретируются как члены эффективного действия, а логарифмические члены относятся к мере интегрирования по $A_{\mu}[58]$.

Очевидно, что в пределе седловой точки эффективное действие совпадает с матричным аналогом действия Намбу-Гото

$$
S_{\mathrm{NG}}=\sqrt{\alpha \beta} \operatorname{tr} \sqrt{G}=\sqrt{\alpha \beta} \operatorname{tr} \sqrt{-\left[A_{\mu}, A_{\nu}\right]^{2}}
$$

Если поля $A_{\mu}$ рассматривать как нульмерные калибровочные потенциалы, то это действие примет вид неабелева действия Борна-Инфельда (НБИ) в пределе сильной связи. Действие Намбу-Гото получается из матричной модели IIB (5.2), (5.3) (или же матричной модели НБИ) уже в приближении квантовой механики с точностью до множителя, относящегося к мере интегрирования, если выбрать специальное значение параметра 
$\gamma=N-1 / 2$. Тогда интегрирование по $Y$ производится точно [63]. Интересно исследовать поправки к этому результату, обусловленные выбором других значений "константы связи" $\gamma$, когда интеграл по матрице $Y$ уже не берется точно, но может быть вычислен пертурбативно по $1 / N$. Тогда предполагается, что параметры $\gamma, \alpha$ и $\beta$ имеют порядок $N$.

Оказывается, что хотя значения $\gamma=N+O(1)$ выделены требованием выполнения общих принципов локальности и репараметризационной инвариантности струнного действия, модель (5.3) интересна также при произвольных $\gamma$, что может отвечать различным сценариям мягкого нарушения репараметризационной инвариантности в рассматриваемой теории.

Уравнения ШД также могут быть решены в модели НБИ [66], но это оказывается излишним, поскольку с помощью этих же уравнений в п. 5.1.2 доказывается точное совпадение модели НБИ и ММК для всех родов после соответствующей замены времен при параметре $\eta$, отличном от нуля $(\eta \sim O(1)$ относительно $N)$.

5.1.1. Матричная модель НБИ в пределе больших $N$. Матричный интеграл [65]-[67], $[98]$

$$
Z_{\mathrm{NBI}}=\int D X \exp \left\{-N \operatorname{tr}\left[X \Lambda+X^{-1}+(2 \eta+1) \ln X\right]\right\}
$$

принадлежит классу ОМК с неполиномиальными потенциалами [44]. Такие модели с отрицательными степенями матрицы $X$ ранее обсуждались в связи с $c=1$ бозонной теорией струн [99], [100]. В работе [65] был развит $\tau$-функциональный подход к таким моделям, в котором параметр $\eta$ играет роль нулевого времени в соответствуюшей интегрируемой иерархии. Кроме того, в конформной точке $\eta=0$ эта модель [65] имеет те же уравнения ШД, что и $U(N)$-модель с внешним полем, которая была решена в рабо$\operatorname{таx~[104],~[45],~[46].~}$

Уравнения ШД для интеграла (5.8) в терминах собственных значений принимают следуюший вид (по индексу $i$ суммирования нет):

$$
\left[-\frac{1}{N^{2}} \lambda_{i} \frac{\partial^{2}}{\partial \lambda_{i}^{2}}-\frac{1}{N^{2}} \sum_{j \neq i} \lambda_{j} \frac{1}{\lambda_{j}-\lambda_{i}}\left(\frac{\partial}{\partial \lambda_{j}}-\frac{\partial}{\partial \lambda_{i}}\right)+\frac{1}{N}(2 \eta-1) \frac{\partial}{\partial \lambda_{i}}+1\right] Z(\lambda)=0
$$

(При $\eta=0$ эти формулы совпадают с соответствуюшими уравнениями ШД $U(N)$-модели [101], [45], [46].) Вычисления, аналогичные MМК, дают ответ в старшем порядке по $N$ :

$$
\begin{aligned}
\ln Z= & N^{2}\left[\left(\eta^{2}+\frac{1}{4}\right) \ln a+\frac{4 \eta^{2}}{\sqrt{a}}-\frac{\eta^{2}}{a}\right]+ \\
& +N \sum_{i}\left[\frac{2}{\sqrt{a}} \sqrt{a \lambda_{i}+\eta^{2}}+\eta \ln \left(\lambda_{i} \frac{\sqrt{a \lambda_{i}+\eta^{2}}-\eta}{\sqrt{a \lambda_{i}+\eta^{2}}+\eta}\right)\right]- \\
& -\frac{1}{2} \sum_{i, j} \ln \left(\sqrt{a \lambda_{i}+\eta^{2}}+\sqrt{a \lambda_{j}+\eta^{2}}\right)+O(1),
\end{aligned}
$$


где параметр $а$ определяется условием связи

$$
1+\frac{1}{2 N} \sum_{j} \frac{1}{\sqrt{a \lambda_{j}+\eta^{2}}}=\frac{1}{\sqrt{a}}
$$

Вычислим теперь условия связи относительно времен Конщевича

$$
t_{k}=\frac{1}{2 k-1} \operatorname{tr} \lambda^{-k+\frac{1}{2}}, \quad k=1,2, \ldots,
$$

в области, в которой такое разложение осмысленно [67].

5.1.2. Уравнения ШД в фазе Концевича. Выпишем уравнение (5.9) через времена Концевича. При этом необходимо учесть нормировочный множитель. Из обших свойств ОМК следует [44], что правильное выражение, не имеюшее явной зависимости от размера матриц $N$, имеет вид

$$
\mathcal{Z}\left(\left\{t_{n}\right\}\right)=\frac{\int D X e^{\Lambda X+V(X)}}{e^{\operatorname{tr} \Lambda X_{0}+V\left(x_{0}\right)} \operatorname{det}^{-\frac{1}{2}}\left(\frac{\delta}{\delta X} \otimes \frac{\delta}{\delta X} V\left(X_{0}\right)\right)},
$$

где $X_{0}$ - стационарная точка действия, $\Lambda+V^{\prime}\left(X_{0}\right)=0$, а детерминант в нормировочном множителе возникает из квазиклассического интегрирования в окрестности стационарной точки.

Введем новые переменные

$$
\lambda_{i}=z_{i}^{2}-\left(\eta+\frac{1}{2}\right)^{2},
$$

в которых нормировочный множитель принимает вид

$$
\exp \left\{-N \sum_{i}\left[2 z_{i}-2 \eta \ln \left(z_{i}+\eta+\frac{1}{2}\right)\right]-\frac{1}{2} \sum_{i, j} \ln \left(z_{i}+z_{j}\right)\right\}
$$

Интеграл (5.12) для модели (5.8) после стандартного интегрирования по Итциксону-Зуберу принимает вид

$$
\mathcal{Z}\left(\left\{t_{n}\right\}\right)=\frac{\operatorname{det}_{\substack{1 \leqslant i \leqslant N \\ 0 \leqslant l \leqslant N-1}}\left\|\xi_{i}^{2 \eta N-l} K_{-2 \eta N-l}\left(N \xi_{i}\right)\right\|}{\prod_{i} e^{-2 N z_{i}}\left(z_{i}+\eta+\frac{1}{2}\right)^{2 \eta N}\left(2 z_{i}\right)^{-\frac{1}{2}} \Delta(z)}, \quad \xi_{i} \equiv \sqrt{\lambda_{i}},
$$

где

$$
K_{\nu}(x)=\int_{-\infty}^{\infty} d s e^{-2 x \operatorname{ch} s+\nu s}
$$

- функции Макдональда (вместо функций Эйри, характерных для ММК).

Введем также новые времена:

$$
t_{n+1}=\frac{1}{2 n-1} \sum_{i} \frac{1}{z_{i}^{2 n-1}}+\delta_{n, 0} \frac{N}{\eta+\frac{1}{2}}, \quad n=0,1, \ldots
$$


получаюшиеся из времен п. 5.1.1 нижнетреугольной заменой, так что эти наборы времен эквивалентны с точки зрения фазовых переходов и критического поведения.

Удобно ввести обозначения КТП, т.е. набор операторов $\left\{\alpha_{k}\right\}_{-\infty}^{\infty}$ :

$$
\begin{aligned}
\left.\alpha_{2 n+1}\right|_{n \geqslant 0} & \equiv \frac{\partial}{\partial t_{n+1}}, \\
\left.\alpha_{-2 n-1}\right|_{n \geqslant 0} & \equiv(2 n+1) t_{n+1}, \\
{\left[\alpha_{-2 k-1}, \alpha_{2 q+1}\right] } & =-(2 k+1) \delta_{k, q},
\end{aligned}
$$

и операторов $V_{q}$ :

$$
V_{q} \equiv \sum_{a, b} \delta_{q, a+b+1}: \alpha_{2 a+1} \alpha_{2 b+1}:+\frac{\delta_{s, 0}}{4}
$$

(нормальное упорядочение : : означает, что все операторы $\alpha_{a}$ с положительными индексами стоят справа от всех операторов $\alpha_{b}$ с отрицательными индексами).

Условия связи на статистическую сумму $\mathcal{Z}\left(\left\{t_{n}\right\}\right)$ имеют вид [67]

$$
L_{s} \mathcal{Z}\left(\left\{t_{n}\right\}\right)=0, \quad s \geqslant-1
$$

где генераторы алгебры Вирасоро

$$
L_{s}=-\left(\eta+\frac{1}{2}\right)^{2} V_{s}+4 \eta N \sum_{k=2}^{\infty} \frac{1}{\left(\eta+\frac{1}{2}\right)^{2 k-3}} \partial_{s+k}
$$

удивительным образом с точностью до члена с одной производной совпадают с условиями связи ММК [47], [38]. Избавиться от этого члена можно, если сдвинуть все старшие времена, оставляя несдвинутыми два первых времени $t_{1}$ и $t_{2}$ :

$$
\tilde{t}_{k} \equiv t_{k}-\frac{4 \eta N}{\left(\eta+\frac{1}{2}\right)^{2 k+1}} \frac{1}{2(2 k+1)}, \quad k \geqslant 3, \quad \tilde{t}_{1,2}=t_{1,2} .
$$

Тогда явное выражение в терминах новых времен можно записать в виде

$$
\begin{aligned}
L_{-1}= & \tilde{t}_{1}^{2}+2 \sum_{m=1}^{\infty}(2 m+1) \tilde{t}_{m+1} \frac{\partial}{\partial \tilde{t}_{m}}-\frac{4 \eta N}{\left(\eta+\frac{1}{2}\right)^{3}} \frac{\partial}{\partial \tilde{t}_{1}} \\
L_{s}= & \sum_{m=1}^{s} \frac{\partial}{\partial \tilde{t}_{m}} \frac{\partial}{\partial \tilde{t}_{s-m}}+2 \sum_{m=1}^{\infty}(2 m-1) \tilde{t}_{m} \frac{\partial}{\partial \tilde{t}_{m+s}}+ \\
& +\frac{1}{4} \delta_{s, 0}-\frac{4 \eta N}{\left(\eta+\frac{1}{2}\right)^{3}} \frac{\partial}{\partial \tilde{t}_{s+2}}, \quad s \geqslant 0,
\end{aligned}
$$

что есть в точности алгебра Вирасоро ММК (3.14). Тем самым доказана эквивалентность модели (5.8) и МMK [9]. 
5.1.3. Выражения для старших родов. Положим константу связи ММК $\gamma=1$. Вклады старших порядков в ММК выражаются через моменты $I_{k}(4.81)$. При $g>1$ вьполнена формула (4.80), которую следует теперь представить через моменты модели (5.8). Введем новые моменты $J_{k}$ :

$$
J_{k}=-(2 k-1) ! ! \frac{1}{N} \sum_{j=1}^{N} \frac{1}{\left(a \lambda_{j}+\eta^{2}\right)^{k+\frac{1}{2}}}, \quad k=0,1,2, \ldots
$$

Интересен лишь закон преобразования для $I_{k}$ при $k>0$, поскольку вся зависимость от момента $I_{0}$ содержится в уравнении связи (4.79). Получим

$$
\left(I_{1}-1\right) \rightarrow a^{\frac{3}{2}}\left(J_{1}+\frac{2}{\eta^{2}}\right), \quad I_{k} \rightarrow a^{k+\frac{1}{2}}\left(J_{k}+\frac{2}{\eta^{2 k}}\right)
$$

и выражение (4.80) тем самым переходит в формулу для разложения по родам модели НБИ:

$$
F_{g}^{\mathrm{NBI}}=\sum_{\sum_{k=2}(k-1) l_{k}=3 g-3}\left\langle\tau_{2}^{l_{2}} \tau_{3}^{l_{3}} \ldots \tau_{3 g-2}^{l_{3 g-2}}\right\rangle_{g} \frac{1}{\left(J_{1}+\frac{2}{\eta^{2}}\right)^{2(g-1)+\sum l_{p}}} \prod_{k=2}^{3 g-2} \frac{\left(J_{k}+\frac{2}{\eta^{2 k}}\right)^{l_{k}}}{l_{k} !}
$$

при $g>1$ и

$$
F_{1}^{\mathrm{NBI}}=\frac{1}{24} \ln \left[a^{\frac{3}{2}}\left(J_{1}+\frac{2}{\eta^{2}}\right)\right] .
$$

Таким образом, выражение (5.10) для рода ноль с учетом нормировочного множителя (5.14) вместе с выражениями (5.28) и (5.27) задают статистическую сумму модели (5.8) для всех родов. Эти выражения, однако, становятся плохо определенными при $\eta \rightarrow 0$, что отвечает случаю $U(N)$-модели, и при $\eta \sim 1 / N$, что отвечает модели [63].

Струнная восприимчивость в пределе больших $N$ получается дифференцированием свободной энергии дважды по струнной константе связи $\eta$. Легко убедиться, что после первого дифференцирования (5.10) условие стационарности все еще выполнено, так что полная производная совпадает с частной производной. В результате получим

$$
\frac{d^{2} \ln Z}{d \eta^{2}}=\frac{\partial^{2} \ln Z}{\partial \eta^{2}}=2 N^{2}(\ln a+3)
$$

5.2. Двухлогарифмическая матричная модель с внешним полем. Исследуем матричный интеграл (2-lnMM), возникаюший в физике, в частности в приложениях к логарифмической модели Казакова-Мигдала [102], [103]:

$$
Z_{2-\ln }=\int D X \exp \{-N \operatorname{tr}[X \Lambda+\alpha \ln (1-X)+\beta \ln (1+X)]\} .
$$

В такой форме этот интеграл имеет наиболее обший вид, поскольку сдвигом и растяжкой полей $X$ и $\Lambda$ можно изменить расположение точек логарифмических особенностей; тем не менее изменить сами константы $\alpha$ и $\beta$ - настоящие заряды модели (5.30) таким образом невозможно. 
Матричный интеграл (5.30), как легко видеть, также относится к классу ОМК и сродни моделям, обсуждавшимся выше. Поэтому для решения этой модели естественно использовать метод уравнений ШД, что и было сделано в работе [68] для произвольных $\alpha$ и $\beta$.

Уравнения ШД для модели (5.30) в терминах собственных значений имеют вид

$$
\begin{array}{r}
{\left[-\frac{1}{N^{2}} \lambda_{i} \frac{\partial^{2}}{\partial \lambda_{i}^{2}}-\frac{1}{N^{2}} \sum_{j \neq i} \lambda_{j} \frac{1}{\lambda_{j}-\lambda_{i}}\left(\frac{\partial}{\partial \lambda_{j}}-\frac{\partial}{\partial \lambda_{i}}\right)+\right.} \\
\left.+\frac{\alpha+\beta-2}{N} \frac{\partial}{\partial \lambda_{i}}+(\beta-\alpha)+\lambda_{i}\right] Z_{2-\ln }(\lambda)=0 .
\end{array}
$$

Остановимся подробнее на способе их решения. Положим

$$
W\left(\lambda_{i}\right)=\frac{1}{N} \frac{\partial}{\partial \lambda_{i}} \ln Z_{2-\ln }, \quad \widetilde{W}(x) \equiv x W(x)-\frac{\alpha+\beta-1}{2}
$$

и введем плотность собственных значений матрицы $\Lambda$

$$
\rho(x)=\frac{1}{N} \sum_{i} \delta\left(x-\lambda_{i}\right),
$$

удовлетворяюшую стандартному условию нормировки

$$
\int d x \rho(x)=1
$$

и переходящую в гладкую функцию в пределе больших $N$.

В пределе больших $N$ производная $W\left(\lambda_{i}\right)$ не дает вклада и оставшиеся члены с учетом нормировочного условия (5.34) дают уравнение для $\widetilde{W}(x)$

$$
\widetilde{W}^{2}(x)+x \int d y \rho(y) \frac{\widetilde{W}(y)-\widetilde{W}(x)}{y-x}=x^{2}+(\beta-\alpha) x+\frac{(\alpha+\beta-1)^{2}}{4},
$$

в котором переменная $\lambda_{i}$ заменена на $x$.

Нелинейное интегральное уравнение (5.35) решается подстановкой комбинации

$$
\widetilde{W}(x)=f(x)+\frac{x}{2} \int d y \frac{\rho(y)}{f(y)} \frac{f(y)-f(x)}{y-x},
$$

в которой $f(x)$ - неизвестная функция, задаваемая подстановкой (5.36) в уравнение (5.35). Асимптотическое поведение $\widetilde{W}(x)$ и $f(x)$ при $x \rightarrow \infty$ следует из уравнения (5.35): $\widetilde{W}(x) \sim x+(\beta-\alpha-1) / 2$, а аналитическое решение с минимальным набором сингулярностей задается функцией

$$
f(x)=\sqrt{a x^{2}+b x+c} .
$$

Введем нулевые моменты внешнего поля:

$$
I_{0}=\int \frac{\rho(x)}{f(x)} d x, \quad J_{0}=\int \frac{\rho(x)}{f(x)} x d x .
$$


Параметры $a, b$ и $c$ однозначно определяются из уравнения (5.35). Сразу получим, что

$$
c=\frac{(\beta+\alpha-1)^{2}}{4}
$$

в то время как параметры $a$ и $b$ должны определяться из следующей функциональной системы уравнений:

$$
\begin{gathered}
1+\frac{1}{2} I_{0}=\frac{1}{\sqrt{a}}, \\
\sqrt{a} J_{0}=\beta-\alpha-\frac{b}{a},
\end{gathered}
$$

или в терминах собственных значений

$$
\begin{gathered}
1+\frac{1}{2 N} \sum_{j} \frac{1}{\sqrt{a \lambda_{j}^{2}+b \lambda_{j}+c}}=\frac{1}{\sqrt{a}}, \\
\sqrt{a} \frac{1}{N} \sum_{j} \frac{\lambda_{j}}{\sqrt{a \lambda_{j}^{2}+b \lambda_{j}+c}}=\beta-\alpha-\frac{b}{a} .
\end{gathered}
$$

Таким образом, получим

$$
W(x)=\frac{\sqrt{a x^{2}+b x+c}}{x}+\frac{1}{2} \int d y \rho(y) \frac{f(y)-f(x)}{f(y)(y-x)}+\frac{\alpha+\beta-1}{2 x} .
$$

Теперь, интегрируя (5.41) по $x$ и проверяя выполнение условий стационарности относительно переменных $a$ и $b$, получим ответ для $2-\ln M М$ в пределе больших $N$ :

$$
\begin{aligned}
\ln Z_{2-\ln }= & N^{2}(\beta-\alpha)^{2}\left[\frac{1}{8} \ln \left(b^{2}-4 a c\right)-\frac{1}{4} \ln a\right]+N^{2}(\beta-\alpha)\left[\frac{1}{4} \ln a-\frac{1}{4} \ln \left(b^{2}-4 a c\right)+\right. \\
& \left.+\sqrt{c} \operatorname{arcth} \frac{2 \sqrt{c a}}{b}-\frac{b}{2 a}\right]+N^{2}\left[\frac{b^{2}}{8 a^{2}}-\frac{c}{2 a}+\frac{2 c}{\sqrt{a}}+\frac{c}{2} \ln \left(b^{2}-4 a c\right)\right]+ \\
& +N \sum_{i}\left[\frac{\alpha+\beta-1}{2} \ln \lambda_{i}+\frac{f\left(\lambda_{i}\right)}{\sqrt{a}}+\frac{1}{2}(\beta-\alpha) \ln \left(\sqrt{a} \lambda_{i}+\frac{b}{2 \sqrt{a}}+f\left(\lambda_{i}\right)\right)-\right. \\
& \left.-\sqrt{c} \operatorname{arcth}\left(\frac{\sqrt{c}+\frac{\lambda_{i} b}{2 \sqrt{c}}}{f\left(\lambda_{i}\right)}\right)\right]-\frac{1}{4} \sum_{i, j}\left[\ln \left(\lambda_{i}-\lambda_{j}\right)+\right. \\
& \left.+\operatorname{arcth}\left(\frac{a \lambda_{i} \lambda_{j}+\frac{\left(\lambda_{i}+\lambda_{j}\right) b}{2}+c}{f\left(\lambda_{i}\right) f\left(\lambda_{j}\right)}\right)\right]+O(1) .
\end{aligned}
$$

Можно проверить явно, что при выполнении условий (5.40)

$$
\frac{\partial}{\partial a} \ln Z_{2-\ln }=\frac{\partial}{\partial b} \ln Z_{2-\ln }=0
$$

и, следовательно,

$$
\frac{1}{N} \frac{\partial}{\partial \lambda_{i}} \ln Z_{2-\ln }=W\left(\lambda_{i}\right)
$$


5.2.1. Условия связи. Произведем сдвиг собственных значений в мастер-уравнении $2-\operatorname{lnMM}\left(\partial_{i} \equiv \partial / \partial \lambda_{i}\right)$ :

$$
\left[-\frac{1}{N^{2}}\left(\lambda_{i}+\xi\right) \partial_{i}^{2}-\frac{1}{N^{2}} \sum_{j \neq i} \frac{\lambda_{j}+\xi}{\lambda_{j}-\lambda_{i}}\left(\partial_{j}-\partial_{i}\right)+\frac{\alpha+\beta-2}{N} \partial_{i}+\beta-\alpha+\lambda_{i}+\xi\right] Z_{2-\ln }=0
$$

При этом учет нормировочного множителя

$$
\prod_{i} \lambda_{i}^{N(\beta-1)} e^{N \lambda_{i}}
$$

который при пронесении через производные дает вклад

$$
\partial_{i} \longrightarrow \partial_{i}+\frac{N(\beta-1)}{\lambda_{i}}+N
$$

в итоге приводит к мастер-уравнению для нормированной статистической суммы

$$
\begin{gathered}
{\left[-\frac{1}{N^{2}}\left(\lambda_{i}+\xi\right) \partial_{i}^{2}-\frac{1}{N^{2}} \sum_{j \neq i} \frac{\lambda_{j}+\xi}{\lambda_{j}-\lambda_{i}}\left(\partial_{j}-\partial_{i}\right)-\frac{2 \lambda_{i}}{N} \partial_{i}+\frac{\alpha-\beta-2 \xi}{N} \partial_{i}-\right.} \\
\left.-\frac{2 \xi(\beta-1)}{N \lambda_{i}} \partial_{i}-\frac{\xi(\beta-1)^{2}}{\lambda_{i}^{2}}+\frac{\beta-1}{\lambda_{i}}\left(\alpha-2 \xi+\frac{\xi}{N} \sum_{j} \frac{1}{\lambda_{j}}\right)\right] \mathcal{Z}_{2-\ln }=0
\end{gathered}
$$

Введем теперь времена 2-lnMM:

$$
t_{n}=\frac{1}{n} \sum_{i} \frac{1}{\lambda_{i}^{n}}
$$

После этого, собирая воедино все множители, стоящие при члене $1 /\left(\lambda_{i}^{k} N^{2}\right)$, сведем уравнения связи для $\mathcal{Z}_{2-\ln }\left(\left\{t_{n}\right\}\right)$, полученные в итоге достаточно объемных вычислений, к следуюшим уравнениям:

$$
L_{k} \mathcal{Z}_{2-\ln }\left(\left\{t_{n}\right\}\right)=0, \quad k \geqslant-1,
$$

где

$$
\begin{aligned}
L_{k}= & V_{k+1}+\xi V_{k}+\xi N(\alpha+\beta-1)\left(\left(1-\delta_{k, 0}-\delta_{k,-1}\right) \frac{\partial}{\partial t_{k}}-N(\beta-1) \delta_{k, 0}\right)+ \\
& +\xi \delta_{k,-1} N(\beta-1)\left(t_{1}-2 N\right) \\
V_{k}= & -\sum_{m=1}^{\infty} m t_{m} \frac{\partial}{\partial t_{m+k}}-\sum_{m=1}^{k-1} \frac{\partial}{\partial t_{m}} \frac{\partial}{\partial t_{k-m}}-N(\alpha-\beta+1)\left(1-\delta_{k, 0}-\delta_{k,-1}\right) \frac{\partial}{\partial t_{k}}+ \\
& +2 N\left(1-\delta_{k,-1}\right) \frac{\partial}{\partial t_{k+1}}+t_{1} \delta_{k,-1} \frac{\partial}{\partial t_{k+1}}+N^{2} \alpha(\beta-1) \delta_{k, 0}
\end{aligned}
$$

В этом выражении производные по $t_{0}$ и $t_{-1}$ несушественны и использованы лишш для сокрашения записи. 
При $k, l \geqslant-1$ генераторы $L_{k}$ удовлетворяют алгебре,

$$
\left[L_{k}, L_{l}\right]=(l-k)\left(L_{k+l+1}+\xi L_{k+l}\right)
$$

Нулевой сдвиг ( $\xi=0)$ привел бы к алгебре Вирасоро-де Витта, в которой бы отсутствовал генератор $L_{-1}$ :

$$
\left[V_{k}, V_{l}\right]=(l-k) V_{k+l}, \quad k, l \geqslant 0 .
$$

Алгебра Вирасоро с минус первым элементом может быть получена из общей алгебры с ненулевым сдвигом с помощью замены

$$
\mathcal{L}_{k}=\sum_{s=0}^{\infty} \frac{(-1)^{s}}{\xi^{s+1}} L_{k+s}, \quad k \geqslant-1,
$$

которая сингулярна при $\xi=0$. Производя эту замену и используя соотношения $\alpha_{\mathrm{KP}}=$ $\beta-1$ и $\varphi=-(\alpha+\beta-1) / 2$, где $\alpha_{\mathrm{KP}}$ - это параметр $\alpha$ ММКП $(2.37)$, получим

$$
\begin{aligned}
\mathcal{L}_{k}= & -\sum_{m=1}^{\infty} m t_{m} \frac{\partial}{\partial t_{m+k}}-\sum_{m=1}^{k-1} \frac{\partial}{\partial t_{m}} \frac{\partial}{\partial t_{k-m}}+2 N \alpha_{\mathrm{KP}} \frac{\partial}{\partial t_{k}}+2 N \frac{\partial}{\partial t_{k+1}}- \\
& -2 \varphi N \sum_{s=1}^{\infty} \frac{1}{(-\xi)^{s}} \frac{\partial}{\partial t_{k+s}}-2 N \alpha_{\mathrm{KP}}\left(\delta_{k, 0}+\delta_{k,-1}\right) \frac{\partial}{\partial t_{k}}-N^{2} \alpha_{\mathrm{KP}}^{2} \delta_{k, 0}+ \\
& +\delta_{k,-1}\left(t_{1}-2 N-\frac{2 \varphi N}{\xi}\right)\left(N \alpha_{\mathrm{KP}}+\frac{\partial}{\partial t_{k+1}}\right) .
\end{aligned}
$$

Возникновение ММКП становится понятным после сдвига времен

$$
t_{n}=\tilde{t}_{n}-2 \varphi \frac{N}{(-\xi)^{n}}+2 N \delta_{n, 1}-\frac{N}{2} \delta_{n, 2}
$$

где

$$
\tilde{t}_{n}=\frac{1}{n} \sum_{i} \frac{1}{\eta_{i}^{n}}
$$

- собственно времена ММКП. После этого сдвига получим

$$
\begin{aligned}
\mathcal{L}_{k}= & -\sum_{m=1}^{\infty} m \tilde{t}_{m} \frac{\partial}{\partial \tilde{t}_{m+k}}-\sum_{m=1}^{k-1} \frac{\partial}{\partial \tilde{t}_{m}} \frac{\partial}{\partial \tilde{t}_{k-m}}+2 N \alpha_{\mathrm{KP}} \frac{\partial}{\partial \tilde{t}_{k}}+N \frac{\partial}{\partial \tilde{t}_{k+2}}- \\
& -2 N \alpha_{\mathrm{KP}}\left(\delta_{k, 0}+\delta_{k,-1}\right) \frac{\partial}{\partial \tilde{t}_{k}}-N^{2} \alpha_{\mathrm{KP}}^{2} \delta_{k, 0}+\tilde{t}_{1} \delta_{k,-1} \frac{\partial}{\partial \tilde{t}_{k+1}}+N \alpha_{\mathrm{KP}} \tilde{t}_{1} \delta_{k,-1}
\end{aligned}
$$

Это в точности алгебра Вирасоро ММКП с нормировочным множителем

$$
\prod_{i} \eta_{i}^{\alpha_{\mathrm{KP}} N} \exp \left\{\frac{N}{2} \eta_{i}^{2}\right\} .
$$


В самом деле, можно произвести ту же операцию в ММКП. Запишем сначала мастер-уравнение для нормированной статистической суммы $\left(\partial_{i} \equiv \partial / \partial \eta_{i}\right)$ :

$$
\left[-\frac{1}{N^{2}} \partial_{i}^{2}-\frac{1}{N^{2}} \sum_{j \neq i} \frac{\partial_{j}-\partial_{i}}{\eta_{j}-\eta_{i}}-\frac{\eta_{i}}{N} \partial_{i}-\frac{2 \alpha_{\mathrm{KP}}}{N \eta_{i}} \partial_{i}+\frac{\alpha_{\mathrm{KP}}}{N \eta_{i}} \sum_{j} \frac{1}{\eta_{j}}-\frac{\alpha_{\mathrm{KP}}^{2}}{\eta_{i}^{2}}\right] \mathcal{Z}_{\mathrm{KP}}=0
$$

Затем, используя времена ММКП $\tilde{t}_{n}$ и собирая все коэффициенты при члене $1 /\left(\eta_{i}^{k} N^{2}\right)$, получим

$$
\mathcal{L}_{k} \mathcal{Z}_{\mathrm{KP}}=0, \quad k \geqslant-1 .
$$

Таким образом, доказана әквивалентность между 2-lnMМ и ММКП в режиме Концевича, т.е. получено точное соотношение между нормированными статистическими суммами двух указанных моделей:

$$
\mathcal{Z}_{2-\ln }\left[\left\{\frac{1}{n} \operatorname{tr} \frac{1}{\lambda^{n}}\right\} ; \alpha, \beta\right]=C(\alpha, \beta) \xi^{2 \varphi(\beta-1) N^{2}} \exp \left[N^{2}(2 \beta-1) \xi\right] \mathcal{Z}_{\mathrm{KP}}\left[\tilde{t}_{n}(\xi, \varphi), \alpha_{\mathrm{KP}}\right],
$$

где

$$
\begin{aligned}
\mathcal{Z}_{2-\ln }\left[\left\{\frac{1}{n} \operatorname{tr} \frac{1}{\lambda^{n}}\right\} ; \alpha, \beta\right] & =\frac{Z_{2-\ln }[\lambda ; \alpha, \beta]}{\prod_{i}\left\{\left(\lambda_{i}-\xi\right)^{N(\beta-1)} \exp \left[N\left(\lambda_{i}-\xi\right)\right]\right\}}, \\
\mathcal{Z}_{\mathrm{KP}}\left[\tilde{t}_{n}(\xi, \varphi), \alpha_{\mathrm{KP}}\right] & =\frac{Z_{\mathrm{KP}}\left[\eta(\xi, \varphi), \alpha_{\mathrm{KP}}\right]}{\prod_{i}\left\{\eta_{i}^{N \alpha_{\mathrm{KP}}} \exp \left(\frac{N}{2} \eta_{i}^{2}\right)\right\}}
\end{aligned}
$$

$\alpha_{\mathrm{KP}}=\beta-1$, a $C(\alpha, \beta)$ - некоторые постоянные, зависящие только от параметров $\alpha$ и $\beta$.

Причина, по которой в формуле (5.64) используется несдвинутое начальное поле $\lambda$ и показана явная зависимость от параметра $\xi$, состоит в том, что для несдвинутого поля $\lambda$ алгебра Вирасоро-де Витта 2-lnMМ не содержит генератор $L_{-1}$. Поэтому возника-

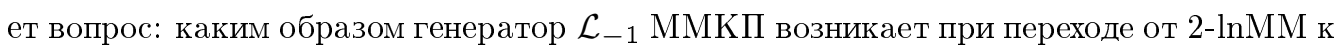
ММКП? Ответ состоит в том, что временной сдвиг (5.56) приводит к тому, что времена ММКП $\tilde{t}_{n}$ становятся зависяшими от $\xi$. Дифференцируя (5.62) по $\xi$ и используя соотношение

$$
\frac{d \tilde{t}_{n}}{d \xi}=(n+1) \tilde{t}_{n+1}-N \delta_{n, 1}
$$

можно получить дополнительное уравнение на статистическую сумму $\mathcal{Z}_{\mathrm{KP}}$

$$
\mathcal{L}_{-1} \mathcal{Z}_{\mathrm{KP}}=0
$$

в котором $\mathcal{L}_{-1}$ и есть недостаюший генератор алгебры Вирасоро ММКП.

5.2.2. Выражения для старших родов. Зададим моменты $2-\ln M M(k \geqslant 0)$ :

$$
\begin{aligned}
& I_{k}^{+}=\frac{1}{N} \sum_{i=1}^{N} \frac{1}{\left(\lambda_{i}-y_{+}\right)^{k+\frac{1}{2}}\left(\lambda_{i}-y_{-}\right)^{\frac{1}{2}}}, \\
& I_{k}^{-}=\frac{1}{N} \sum_{i=1}^{N} \frac{1}{\left(\lambda_{i}-y_{+}\right)^{\frac{1}{2}}\left(\lambda_{i}-y_{-}\right)^{k+\frac{1}{2}}},
\end{aligned}
$$

3 Теоретическая и математическая физика, т. 127, № 2, 2001 г. 
где

$$
y_{ \pm}=-\frac{b}{2 a} \pm \sqrt{\frac{b^{2}}{4 a^{2}}-\frac{c}{a}} .
$$

Помимо переменной $\varphi=-(\alpha+\beta-1) / 2=\sqrt{c}$ удобно ввести переменную $\gamma=(\beta-\alpha) / 2$. Задача состоит в том, чтобы найти соотношение между моментами $I_{k}^{ \pm}$и $M_{k}(4.93)$, $J_{k}(4.94)$ указанных моделей при $k \geqslant 0$ (для $k=0$ соотношение следует из уравнений связи (4.75) и (4.76)). Производя сдвиг собственных значений $\left(y_{ \pm}=x_{ \pm}+\xi\right)$ и делая замену времен, получим $(k \geqslant 1)$

$$
M_{k}=I_{k}^{+}+2 \varphi \frac{(-1)^{k+1}}{y_{+}^{k+\frac{1}{2}} y_{-}^{\frac{1}{2}}}, \quad J_{k}=I_{k}^{-}+2 \varphi \frac{(-1)^{k+1}}{y_{+}^{\frac{1}{2}} y_{-}^{k+\frac{1}{2}}} .
$$

Таким образом, для 2-lnMМ имеем

$$
\ln \mathcal{Z}_{2-\ln }=\sum_{g=0}^{\infty} N^{2-2 g} F_{g}^{2-\ln },
$$

откуда с использованием выражения (4.18) для свободной энергии ММКП получим

$$
\begin{aligned}
F_{g}^{2-\ln }= & \sum_{\alpha_{j}>1, \beta_{j}>1}\left\langle\alpha_{1} \ldots \alpha_{s} ; \beta_{1} \ldots \beta_{l} \mid \alpha \beta \gamma\right\rangle_{g} \prod_{i=1}^{s}\left(I_{\alpha_{i}}^{+}+2 \varphi \frac{(-1)^{\alpha_{i}+1}}{y_{+}^{\alpha_{i}+\frac{1}{2}} y_{-}^{\frac{1}{2}}}\right) \times \\
& \times \prod_{i=1}^{l}\left(I_{\beta_{i}}^{-}+2 \varphi \frac{(-1)^{\beta_{i}+1}}{y_{+}^{\frac{1}{2}} y_{-}^{\beta_{i}+\frac{1}{2}}}\right)\left\{\left(I_{1}^{+}+2 \varphi \frac{1}{y_{+}^{\frac{3}{2}} y_{-}^{\frac{1}{2}}}\right)^{\alpha} \times\right. \\
& \left.\times\left(I_{1}^{-}+2 \varphi \frac{1}{y_{+}^{\frac{1}{2}} y_{-}^{\frac{3}{2}}}\right)^{\beta}\left(y_{+}-y_{-}\right)^{\gamma}\right\}^{-1}, g>1, \\
F_{1}= & -\frac{1}{24} \ln \left\{\left(I_{1}^{+}+2 \varphi \frac{1}{y_{+}^{\frac{3}{2}} y_{-}^{\frac{1}{2}}}\right)\left(I_{1}^{-}+2 \varphi \frac{1}{y_{+}^{\frac{1}{2}} y_{-}^{\frac{3}{2}}}\right)\left(y_{+}-y_{-}\right)^{4}\right\} .
\end{aligned}
$$

Вычислим теперь струнную восприимчивость по отношению к зарядам $\gamma$ и $\varphi$ для выражения (5.42). Из уравнения (5.43) получим, что $d \ln Z / d \gamma=\partial \ln Z / \partial \gamma$, и то же самое верно для $\varphi$. Более того, удивительное обстоятельство состоит в том, что полученные выражения сами оказываются стационарными по отношению к полному дифференцированию по $a$ и $b$. Поэтому полные вторые производные (в том числе смешанные) по $\gamma$ и $\varphi$ совпадают с соответствуюшими частными производными - свойство, присушее всем исследуемым в данной работе моделям. Таким образом, имеем

$$
\begin{aligned}
& \chi_{1}=\frac{1}{N^{2}} \frac{d^{2}}{d \gamma^{2}} \ln Z=\ln \left(b^{2}-4 a c\right)-2 \ln a, \\
& \chi_{2}=\frac{1}{N^{2}} \frac{d^{2}}{d \gamma d \varphi} \ln Z=-2 \operatorname{arcth} \frac{2 \sqrt{a c}}{b}=-\ln \frac{b+2 \sqrt{a c}}{b-2 \sqrt{a c}}, \\
& \chi_{3}=\frac{1}{N^{2}} \frac{d^{2}}{d \varphi^{2}} \ln Z=\ln \left(b^{2}-4 a c\right)+6 .
\end{aligned}
$$

Сравнивая со струнной восприимчивостью ММКП (4.101), получим

$$
\chi_{\mathrm{KP}}=\chi_{1} \text {. }
$$




\section{6. ТОЧНЫЕ СООТНОШЕНИЯ МЕЖДУ МАТРИЧНЫМИ ИНТЕГРАЛАМИ КОНЦЕВИЧА-ПЕННЕРА И КОНЦЕВИЧА}

Обсудим соотношения между ММК (2.17) и ММКП (2.37). В этом разделе предполагается, что асимптотическое разложение модели (2.37) производится по временам ДПМ $T_{2 n}^{ \pm}$. Как и в случае MMK, утверждение об отсутствии зависимости от нечетных времен можно доказать непосредственно, анализируя уравнения связи.

Соотношения ДСП обусловливаются некоторой предельной процедурой, при которой, очевидно, теряется значительная часть информации, заключенной в модели (2.37). Покажем, однако, что существует точное соотношение, связывающее ММКП (2.37) и ММК (2.17).

6.1. Соотношение между моментами и переменными ДПМ. Выразим (4.18) через величины из правой части формулы (2.35). Для этого перепишем как моменты $M_{k}, J_{k}$, так и уравнения связи $(4.75),(4.76)$ через переменные $\lambda$, где $\eta=\sqrt{\alpha}\left(e^{\lambda}+e^{-\lambda}\right)$. Тогда для концевых точек разреза получим

$$
x=2 \sqrt{\alpha}+\xi, \quad y=-2 \sqrt{\alpha}+\beta,
$$

где $\xi$ и $\beta$ сами являются некоторыми полиномами от старших моментов $M_{i}$ и $J_{i} \mathrm{c} i, j \geqslant 0$. Тогда, например, получим, что моменты $M_{k}$ даются следующими выражениями:

$$
M_{k}=\frac{1}{N} \operatorname{tr} \frac{\left(e^{\lambda}\right)^{k+1}}{\sqrt{\alpha} k+1\left(\left(e^{\lambda}-1\right)^{2}-\frac{\xi}{\sqrt{\alpha}} e^{\lambda}\right)^{k+\frac{1}{2}}\left(\left(e^{\lambda}+1\right)^{2}-\frac{\beta}{\sqrt{\alpha}} e^{\lambda}\right)^{\frac{1}{2}}}-\delta_{k, 1} .
$$

(Для $J_{k}$ выражение точно такое же с точностью до перестановки показателей степеней $k+1 / 2$ и $1 / 2$ двух членов в знаменателе.)

В формуле (6.2) разложение идет по членам

$$
H_{a b}=\frac{1}{N} \operatorname{tr} \frac{e^{(a+1) \lambda}}{\left(e^{\lambda}-1\right)^{2 a+1}} \frac{e^{b \lambda}}{\left(e^{\lambda}+1\right)^{2 b+1}},
$$

где $b \geqslant 0, a \geqslant k . H_{a b}$ представляется как линейная сумма слагаемых

$$
L_{a}=\frac{1}{N} \operatorname{tr} \frac{\partial^{a}}{\partial \lambda^{a}} \frac{1}{e^{\lambda}-1}, \quad R_{b}=\frac{1}{N} \operatorname{tr} \frac{\partial^{b}}{\partial \lambda^{b}} \frac{1}{e^{\lambda}+1},
$$

где суммирование ведется лишь по четным степеням производных по $\lambda$ :

$$
H_{a b}=\sum_{i=0}^{a} \alpha_{a b}^{i} L_{2 i}+\sum_{j=0}^{b-1} \beta_{a b}^{j} R_{2 j} .
$$

Это утверждение прямо следует из симметрийных свойств $H_{a b}$ :

$$
\begin{aligned}
H_{a b}(-\lambda) & =-H_{a b}(\lambda), \\
L_{i}(-\lambda) & =(-1)^{i+1} L_{i}(\lambda)-\delta_{i, 0}, \\
R_{i}(-\lambda) & =(-1)^{i+1} R_{i}(\lambda)+\delta_{i, 0} .
\end{aligned}
$$

Тем самым мы доказали следуюшую лемму (связанную с теоремой 4): 
Лемма. Статистическая сумма матричной модели $(2.37), \mathcal{F}_{\mathrm{KP}}\left(\left\{T^{ \pm}\right\}\right)$, зависит только от четньх времен

$$
T_{2 n}^{ \pm}=\frac{1}{(2 n+1) !} \operatorname{tr} \frac{\partial^{2 n}}{\partial \lambda^{2 n}} \frac{1}{e^{\lambda} \pm 1} .
$$

Удерживая только члены нулевого и первого порядков по следам матрицы $\lambda$ в выражениях для моментов, получим

$$
\begin{aligned}
M_{k} & \sim \frac{1}{\sqrt{\alpha} k+1} \frac{1}{N} \operatorname{tr} \frac{e^{\lambda(k+1)}}{\left(e^{\lambda}-1\right)^{2 k+1}\left(e^{\lambda}+1\right)}+\delta_{k 1}, \\
J_{k} & \sim \frac{1}{\sqrt{\alpha} k+1} \frac{1}{N} \operatorname{tr} \frac{e^{\lambda(k+1)}}{\left(e^{\lambda}-1\right)\left(e^{\lambda}+1\right)^{2 k+1}}+\delta_{k 1}, \\
d & \sim \sqrt{\alpha}\left\{4-\frac{1}{\alpha} \frac{1}{N} \operatorname{tr} \frac{2}{\left(e^{\lambda}-1\right)\left(e^{\lambda}+1\right)}\right\} .
\end{aligned}
$$

Члены, остающиеся в ДСП, - это в точности члены, получающиеся из членов без редукций из левой части формулы (2.35). Таким образом,

$$
\left\langle\left\langle\tau_{d_{1}} \ldots \tau_{d_{n}}\right\rangle\right\rangle_{g}=\left\langle\tau_{d_{1}} \ldots \tau_{d_{n}}\right\rangle_{g}
$$

для $d_{1}+\cdots+d_{n}=3 g-3+n$.

6.2. Алгебра времен $t_{2 n}^{ \pm}$. Статистическая сумма модели (2.37) выражается через времена

$$
t_{k}^{ \pm}=\frac{1}{N} \operatorname{tr} \frac{1}{(k+1) !} \frac{\partial^{k}}{\partial \lambda^{k}} \frac{1}{e^{\lambda} \pm 1}
$$

Введем также времена $t_{k}^{ \pm}(\lambda)$, являющиеся вариационными производными $t_{k}^{ \pm}$:

$$
t_{k}^{ \pm}\left(\lambda_{j}\right)=\frac{1}{k !} \frac{\partial^{k}}{\partial \lambda_{j}^{k}} \frac{1}{e^{\lambda_{j}} \pm 1} .
$$

Формула разложения $1 /\left(e^{\lambda}-1\right)$ имеет стандартный вид:

$$
\frac{1}{e^{\lambda}-1}=\frac{1}{\lambda}-\frac{1}{2}+\sum_{m=0}^{\infty} \frac{B_{2 m+2}}{(2 m+2) !} \lambda^{2 m+1},
$$

где $B_{m}$ - числа Бернулли. Разложение $1 /\left(e^{\lambda}+1\right)$ имеет такой же вид в окрестности полюса $\lambda=i \pi$, но нам также понадобится разложение этого выражения по $\lambda$ в его несингулярной нулевой точке. Поскольку

$$
\frac{1}{e^{\lambda}+1}=\frac{1}{e^{\lambda}-1}-\frac{2}{e^{2 \lambda}-1}
$$

получим

$$
\frac{1}{e^{\lambda}+1}=\frac{1}{2}+\sum_{m=0}^{\infty} \frac{B_{2 m+2}}{(2 m+2) !}\left(1-2^{2 m+2}\right) \lambda^{2 m+1} .
$$


Вычисляя производные от этих выражений, получим, что все нечетные времена строго симметричны при изменении знака $\lambda \rightarrow-\lambda$ :

$$
t_{k}^{ \pm}(-\lambda)=t_{k}^{ \pm}(\lambda)(-1)^{k+1} \pm \delta_{k, 0}
$$

Найдем теперь “правила слияния” для времен $t_{k}^{ \pm}(\lambda)$, приняв во внимание тот факт, что “отрицательные" времена $t_{k}^{-}$содержат только чистые полюсы $(k+1)$-го порядка по $\lambda$. Отсюда сразу получим, что для нечетных времен

$$
\begin{aligned}
t_{2 n+1}^{ \pm}(\lambda) t_{2 m+1}^{ \pm}(\lambda)= & \pm t_{2(n+m)+3}^{ \pm}(\lambda) \mp \sum_{k=0}^{m} \frac{B_{2(n+m-k+1)}}{2(n+m-k+1)} \frac{t_{2 k+1}^{ \pm}(\lambda)}{(2 m-2 k) !(2 n+1) !} \mp \\
& \mp \sum_{p=0}^{n} \frac{B_{2(n+m-p+1)}}{2(n+m-p+1)} \frac{t_{2 p+1}^{ \pm}(\lambda)}{(2 n-2 p) !(2 m+1) !}
\end{aligned}
$$

и для смешивающих соотношений

$$
\begin{aligned}
t_{2 n+1}^{-}(\lambda) t_{2 m+1}^{+}(\lambda)= & -\sum_{k=0}^{n} \frac{B_{2(n+m-k+1)}}{2(n+m-k+1)} \frac{2^{2(n+m-k+1)}-1}{(2 n-2 k) !(2 m+1) !} t_{2 k+1}^{-}(\lambda)+ \\
& +\sum_{k=0}^{m} \frac{B_{2(n+m-k+1)}}{2(n+m-k+1)} \frac{2^{2(n+m-k+1)}-1}{(2 m-2 k) !(2 n+1) !} t_{2 k+1}^{+}(\lambda)
\end{aligned}
$$

6.3. Уравнения Швингера-Дайсона в терминах времен $t_{2 n}^{ \pm}$. Начнем с уравнений ШД (3.21) для интеграла (3.18), которые в терминах $\lambda_{i}$ имеют вид

$$
\begin{gathered}
\left\{\frac{1}{\left(\frac{\alpha N}{2}\right)^{2}}\left[\frac{1}{e^{\lambda_{j}}-e^{-\lambda_{j}}} \vec{\partial}_{j} \frac{1}{e^{\lambda_{j}}-e^{-\lambda_{j}}} \vec{\partial}_{j}+\sum_{i \neq j} \frac{\frac{1}{e^{\lambda_{j}}-e^{-\lambda_{j}}} \vec{\partial}_{j}-\frac{1}{e^{\lambda_{i}-e^{-\lambda_{i}}}+\vec{\partial}_{i}}}{\vec{\lambda}_{i}-\left(e^{\lambda_{i}}+e^{-\lambda_{i}}\right)}\right]+\right. \\
\left.+\left(1-\frac{2}{\alpha}\right)-\frac{2}{\alpha N}\left[e^{\lambda_{j}}+e^{-\lambda_{j}}\right] \frac{1}{e^{\lambda_{j}}-e^{-\lambda_{j}}} \vec{\partial}_{j}\right\} \mathcal{Z}_{N}\left(e^{\lambda}\right)=0, \quad \vec{\partial}_{j} \equiv \frac{\partial}{\partial \lambda_{j}}
\end{gathered}
$$

Найдем набор уравнений для интеграла $\mathcal{Z}_{\mathrm{KP}}(\lambda)$, отличающегося от $\mathcal{Z}_{N}(\lambda)$ на нормировочный множитель:

$$
\mathcal{Z}_{N}(\lambda)=\mathcal{Z}_{\mathrm{KP}}(\lambda) \prod_{i, j=1}^{N}\left(e^{\lambda_{i}+\lambda_{j}}-1\right)^{-\frac{1}{2}} \exp \left\{-N\left(\frac{\alpha}{2}-1\right) \sum_{i=1}^{N} \lambda_{i}+\frac{\alpha N}{4} \sum_{i=1}^{N} e^{2 \lambda_{i}}\right\}
$$

Коммутируя дополнительные сомножители с дифференциалами в (6.15), мы получим уравнения ШД в терминах $\lambda_{i}$. Они достаточно громоздки и здесь не приводятся, заметим лишь, что они опять содержат члены с двумя, одной и без производных по $\lambda_{i}$. После длинных вычислений их можно частично переписать в терминах времен (6.9) (см. [104]) и выделить из них часть, стоящую при некотором выбранном $t_{2 k+1}^{ \pm}\left(\lambda_{j}\right)$. Каждая такая 
часть порождает некоторую линейно (но не алгебраически) независимую связь, накладываемую на $\mathcal{Z}_{\mathrm{KP}}(\lambda)$. Точно так же, как и в случае MМК, единственный действительно нетривиальный расчет связан со средним членом, содержащим одну производную, который появляется из “интегрального" члена уравнений ШД. Рассмотрим этот случай более подробно.

Начнем с тождества

$$
\frac{1}{1-e^{-x-y}}\left(1+\partial_{x}+\partial_{y}\right)^{2 k+1} \frac{1}{\left(e^{x} \pm 1\right)\left(e^{y} \pm 1\right)}=\frac{1}{e^{y}-e^{x}}\left(\partial_{y}-\partial_{x}\right)^{2 k+1} \frac{ \pm e^{x+y}}{\left(e^{x} \pm 1\right)\left(e^{y} \pm 1\right)},
$$

которое обусловлено симметриями $x \rightarrow-x$ и $y \rightarrow-y$, выполненными по отдельности для $x$ и $y$. В левой части (6.17) получим

$$
\begin{aligned}
& \frac{1}{1-e^{-x-y}}\left(1+\partial_{x}+\partial_{y}\right)^{2 k+1} \frac{1}{\left(e^{x} \pm 1\right)\left(e^{y} \pm 1\right)}= \\
& \quad=\frac{1}{e^{x}-e^{-y}}\left(\partial_{y}+\partial_{x}\right)^{2 k+1} \frac{ \pm e^{x-y}}{\left(e^{x} \pm 1\right)\left(e^{y} \pm 1\right)}=\sum_{n, m} d_{n, m}^{k} t_{2 n+1}^{ \pm}(x) t_{2 m+1}^{ \pm}(y),
\end{aligned}
$$

где $d_{n, m}^{k} \in \mathbb{C}$ - некоторые пока неизвестные коэффициенты. Умножая правую часть (6.17) и левую часть (6.18) соответственно на $e^{y}-e^{x}$ и $e^{y}-e^{-x}$, чтобы устранить множители перед членами с производными, и вычитая одно выражение из другого, получим

$$
\begin{aligned}
\sum_{n, m} d_{n, m}^{k}\left(e^{y}-e^{-y}\right) t_{2 n+1}^{ \pm}(y) t_{2 m+1}^{ \pm}(x)= & \left(\partial_{y}-\partial_{x}\right)^{2 k+1}\left[-\frac{1}{e^{x} \pm 1}-\frac{e^{x}}{\left(e^{x} \pm 1\right)\left(e^{y} \pm 1\right)}\right]+ \\
& +\left(\partial_{y}+\partial_{x}\right)^{2 k+1} \frac{e^{x}}{\left(e^{x} \pm 1\right)\left(e^{y} \pm 1\right)}
\end{aligned}
$$

Отсюда найдем, что

$$
\begin{aligned}
& -\sum_{\substack{k=0 \\
( \pm)}}^{\infty} \sum_{i=1}^{N} \frac{1}{\left(e^{\lambda_{i}}-e^{-\lambda_{i}}\right)\left(1-e^{-\lambda_{i}-\lambda_{j}}\right)}\left[1+\frac{\partial}{\partial \lambda_{i}}+\frac{\partial}{\partial \lambda_{j}}\right]^{2 k+1} \frac{1}{\left(e^{\lambda_{i}} \pm 1\right)\left(e^{\lambda_{j}} \pm 1\right)}= \\
& = \pm \sum_{i=1}^{N} \frac{2}{\left(e^{\lambda_{i}}-e^{-\lambda_{i}}\right)^{2}} \sum_{n=1}^{k}\left(\begin{array}{c}
2 n \\
2 k+1
\end{array}\right)\left(\frac{\partial}{\partial \lambda_{i}}\right)^{2 n} \frac{1}{e^{\lambda_{i}} \pm 1}\left(\frac{\partial}{\partial \lambda_{j}}\right)^{2(k-n)+1} \frac{1}{e^{\lambda_{j}} \pm 1} \pm \\
& \quad \pm\left(\frac{\partial}{\partial \lambda_{j}}\right)^{2 k+1} \frac{1}{e^{\lambda_{j}} \pm 1}\left(\frac{3}{2} t_{2}^{ \pm}-\frac{1}{8}\left(t_{0}^{+}+t_{0}^{-}\right)\right) .
\end{aligned}
$$

Собирая вместе все искомые члены, получим набор условий на $e^{\mathcal{F}_{\mathrm{KP}}(\lambda)} \equiv \mathcal{Z}_{\mathrm{KP}}(\lambda)$, который имеет вид системы уравнений

$$
\sum_{k=0}^{\infty} t_{2 k+1}^{+}\left(\lambda_{j}\right)\left(L_{2 k+1}^{+} \mathcal{Z}_{\mathrm{KP}}(\lambda)\right)+\sum_{k=0}^{\infty} t_{2 k+1}^{-}\left(\lambda_{j}\right)\left(L_{2 k+1}^{-} \mathcal{Z}_{\mathrm{KP}}(\lambda)\right)=0
$$


выполненной при всех $j$. В асимптотическом пределе мы можем считать следы от всех степеней матрицы $\Lambda$ независимыми.

Все времена (6.9) интерпретируются как независимые переменные. Подобно ММК, времена $\left\{t_{k}^{+}\right\}$(и $\left\{t_{k}^{-}\right\}$) становятся независимыми только при $N \rightarrow \infty$. Более того, имеются соотношения, смешиваюшие эти два набора времен, выполненные (по крайней мере, формально) для всех $N$. Например, учитывая структуру полюсов времен (6.9) по переменным $\lambda$, получим

$$
t_{0}^{+}=\operatorname{tr} \frac{1}{e^{\lambda}+1}=-\operatorname{tr} \frac{1}{e^{\lambda+i \pi}-1}=-\sum_{k=0}^{\infty}(k+1)(i \pi)^{k} t_{k}^{-} .
$$

Заметим, однако, что такие выражения всегда содержат бесконечные суммы, а поскольку ответ (2.35) при конечных $g$ и $n$ - конечный полином, то его вид однозначно определяется в терминах времен $\left\{t_{2 k}^{ \pm}\right\}$(однозначность следует из анализа структуры полюсов в точках $i \pi n)$. Тем самым в асимптотическом разложении по $N$ и $\alpha$ все эти времена могут рассматриваться как независимые переменные. Соответственно все $L_{2 k+1}^{ \pm}$- независимые генераторы алгебры связей для $\mathcal{Z}_{\mathrm{KP}}(\lambda)$. Явный вид $L_{2 k+1}^{ \pm}$достаточно громоздок и не приводится здесь. Более того, как будет показано, этот ответ сам по себе и не нужен.

6.4. Алгебра связей $L_{2 s+1}^{ \pm}$. Ответ для коммутационных соотношений генераторов $L_{2 s+1}^{ \pm}$получается прямым вычислением:

$$
\left[L^{+}, L^{-}\right] \equiv 0
$$

Таким образом, хотя как генераторы $L_{.}^{+}$, так и $L^{-}$. содержат производные и по временам “ $t^{+}$”, и по временам " $t$ "”, две половины алгебры расцепляются.

Рассмотрим для определенности алгебру $L_{.}^{+}$:

$$
\left[L_{2 s+1}^{+}, L_{2 t+1}^{+}\right]=\frac{4(s-t)}{\alpha^{2}}\left(L_{2 s+2 t-1}^{+}-\sum_{m=0}^{\infty} \frac{B_{2 m+2}}{2 m+2} \frac{2^{2 m+2}}{(2 m) !} L_{2(s+t+m)+1}^{+}\right) .
$$

После верхнетреугольного преобразования генераторов

$$
\tilde{L}_{s}^{ \pm}=\sum_{k=0}^{\infty} \frac{2^{2 k+1}}{(2 k+2) !} L_{2(s+k)+3}^{ \pm}, \quad s \geqslant-1,
$$

она становится обычной алгеброй Вирасоро:

$$
\begin{array}{ll}
{\left[\tilde{L}_{s}^{ \pm}, \tilde{L}_{t}^{ \pm}\right]=\frac{4}{\alpha^{2}}(s-t) \tilde{L}_{s+t}^{ \pm},} & s, t \geqslant-1 \\
{\left[\tilde{L}_{s}^{+}, \tilde{L}_{t}^{-}\right]=0,} & s, t \geqslant-1 .
\end{array}
$$

Найдем преобразование времен, отвечающее замене генераторов (6.24). Условия связей (6.21) в преобразованных временах становятся половинами двух алгебр Вирасоро:

$$
\sum_{k=0}^{\infty} \tilde{t}_{k}^{+}\left(\lambda_{j}\right)\left(\tilde{L}_{k-1}^{+} \mathcal{Z}_{\mathrm{KP}}(\lambda)\right)+\sum_{k=0}^{\infty} \tilde{t}_{k}^{-}\left(\lambda_{j}\right)\left(\tilde{L}_{k-1}^{-} \mathcal{Z}_{\mathrm{KP}}(\lambda)\right)
$$


где

$$
\tilde{t}_{n}^{+}(\lambda)=t_{2 n+1}^{+}(\lambda)-\sum_{k=0}^{n-1} \frac{B_{2 k+2}}{2 k+2} \frac{2^{2 k+2}}{(2 k) !} t_{2 n-2 k-1}^{+}(\lambda), \quad n \geqslant 0,
$$

или в терминах многочленов Бернулли

$$
\tilde{t}_{n}^{ \pm}(\lambda)=\frac{2^{2 n+1}}{(2 n+1) !}\left[(2 n+1) \frac{\partial}{2 \partial \lambda} B_{2 n}\left(\frac{\partial}{2 \partial \lambda}\right)-2 n B_{2 n+1}\left(\frac{\partial}{2 \partial \lambda}\right)\right] \frac{1}{e^{\lambda} \pm 1}
$$

где

$$
B_{n}(x)=\sum_{s=0}^{n}\left(\begin{array}{l}
s \\
n
\end{array}\right) B_{s} x^{n-s},
$$

$B_{0}=1, \quad B_{1}=-1 / 2, \quad B_{2}=1 / 6, \quad B_{4}=-1 / 30, \quad B_{6}=1 / 42, \quad B_{8}=-1 / 30, \quad B_{10}=5 / 66$ и т.д.; $B_{2 n+1}=0$ при $n>0$.

Генераторы $\tilde{L}_{s}^{+}, s \geqslant 0$, имеют вид

$$
\begin{aligned}
\tilde{L}_{s}^{+}= & \frac{1}{\alpha^{2} N^{2}}\left\{\sum_{m=0}^{s-1} \frac{\partial}{\partial t_{2 m}^{+}} \frac{\partial}{\partial t_{2(s-m-2)}^{+}}-\right. \\
& -2 \sum_{n, m=0}^{\infty} \frac{B_{2(n+m+1)}}{2(n+m+1)} \frac{1}{(2 n+1) !(2 m) !} \frac{\partial}{\partial t_{2 n}^{+}} \frac{\partial}{\partial t_{2(m+s)}^{+}}+ \\
& \left.+2 \sum_{n, m=0}^{\infty} \frac{B_{2(n+m+1)}}{2(n+m+1)} \frac{2^{2(n+m+1)}-1}{(2 n+1) !(2 m) !} \frac{\partial}{\partial t_{2 n}^{-}} \frac{\partial}{\partial t_{2(m+s)}^{+}}\right\}+ \\
& +\frac{2}{\alpha} \sum_{k=0}^{\infty} \frac{2^{2 k+1}}{(2 k+2) !} \frac{\partial}{\partial t_{2(s+k+1)}^{+}}+\frac{2}{\alpha^{2}} \sum_{n=0}^{\infty}(2 n+1) t_{2 n}^{+} \frac{\partial}{\partial t_{2(n+s)}^{+}}+\frac{\delta_{s, 0}}{4 \alpha^{2}}, \quad s \geqslant 0 .
\end{aligned}
$$

Как и в струнном уравнении, генератор $\tilde{L}_{-1}^{+}$имеет специальный вид:

$$
\begin{aligned}
\tilde{L}_{-1}^{+}= & \frac{1}{\alpha^{2} N^{2}}\left\{-2 \sum_{n, m=0}^{\infty} \frac{B_{2(n+m+2)}}{2(n+m+2)} \frac{1}{(2 n+1) !(2 m+2) !} \frac{\partial}{\partial t_{2 n}^{+}} \frac{\partial}{\partial t_{2 m}^{+}}+\right. \\
& \left.+2 \sum_{n, m=0}^{\infty} \frac{B_{2(n+m+2)}}{2(n+m+2)} \frac{2^{2(n+m+2)}-1}{(2 n+1) !(2 m+2) !} \frac{\partial}{\partial t_{2 n}^{-}} \frac{\partial}{\partial t_{2 m}^{+}}\right\}+ \\
& +\frac{2}{\alpha} \sum_{k=0}^{\infty} \frac{2^{2 k+1}}{(2 k+2) !} \frac{\partial}{\partial t_{2 k}^{+}}+\frac{2}{\alpha^{2}} \sum_{n=0}^{\infty}(2 n+3) t_{2 n+2}^{+} \frac{\partial}{\partial t_{2 n}^{+}}+ \\
& +\frac{N^{2}}{\alpha^{2}}\left[t_{0}^{+}-\frac{1}{N^{2}} \sum_{n=0}^{\infty} \frac{B_{2 n+2}}{(2 n+2) !}\left(\frac{\partial}{\partial t_{2 n}^{+}}-\left(2^{2 n+2}-1\right) \frac{\partial}{\partial t_{2 n}^{-}}\right)\right]^{2} .
\end{aligned}
$$

Генераторы $\tilde{L}_{s}^{-}$получаются из $\tilde{L}_{s}^{+}$при взаимных заменах $t_{2 s}^{+} \leftrightarrow-t_{2 s}^{-}$и $\partial / \partial t_{2 s}^{+} \leftrightarrow$ $-\partial / \partial t_{2 s}^{-}$. Все производные предполагаются действующими направо (это, в частности, исключает постоянный член в $L_{-1}^{ \pm}$). 
Производя каноническое преобразование $\mathcal{A}$ (явный вид которого см. (6.37)) времен и их производных

$$
\begin{gathered}
\widehat{t_{2 n}^{ \pm}}=e^{-\mathcal{A}} t_{2 n}^{ \pm} e^{\mathcal{A}}=t_{2 n}^{ \pm}-\frac{1}{N^{2}} \sum_{m=0}^{\infty} \frac{B_{2(n+m+1)}}{2(n+m+1)} \frac{1}{(2 n+1) !(2 m+1) !} \times \\
\times\left(\frac{\partial}{\partial t_{2 m}^{ \pm}}-\left(2^{2(n+m+1)}-1\right) \frac{\partial}{\partial t_{2 m}^{\mp}}\right) \pm\left(1-\delta_{n, 0}-\delta_{n, 1}\right) \alpha \frac{2^{2 n-1}}{(2 n+1) !}, \\
\frac{\partial}{\partial t_{2 n}^{ \pm}}=e^{-\mathcal{A}} \frac{\partial}{\partial t_{2 n}^{ \pm}} e^{\mathcal{A}}=\frac{\partial}{\partial t_{2 n}^{ \pm}}
\end{gathered}
$$

и делая масштабное преобразование времен

$$
\xi_{n}^{ \pm}=N t_{2 n}^{\widehat{ \pm}}, \quad \frac{\partial}{\partial \xi_{n}^{ \pm}}=N^{-1} \frac{\partial}{\partial \widehat{t_{2 n}^{ \pm}}},
$$

выразим генераторы Вирасоро (6.30) и (6.29) в терминах $\xi^{ \pm}$:

$$
\begin{aligned}
\mathcal{L}_{-1}^{ \pm}= & \frac{\alpha^{2}}{4} \tilde{L}_{-1}^{ \pm}=\frac{1}{2} \sum_{n=0}^{\infty}(2 n+3) \xi_{n+1}^{ \pm} \frac{\partial}{\partial \xi_{n}^{ \pm}}+\frac{\xi_{0}^{ \pm 2}}{4}+\frac{\alpha N}{2} \frac{\partial}{\partial \xi_{0}^{ \pm}}, \\
\mathcal{L}_{s}^{ \pm}= & \frac{\alpha^{2}}{4} \tilde{L}_{s}^{ \pm}=\frac{1}{4} \sum_{m=0}^{s-1} \frac{\partial}{\partial \xi_{m}^{ \pm}} \frac{\partial}{\partial \xi_{s-m-1}^{ \pm}}+\frac{1}{2} \sum_{n=0}^{\infty}(2 n+1) \xi_{n}^{ \pm} \frac{\partial}{\partial \xi_{n+s}^{ \pm}}+ \\
& +\frac{\alpha N}{2} \frac{\partial}{\partial \xi_{s+1}^{ \pm}}+\frac{\delta_{s, 0}}{16}, \quad s \geqslant 0 .
\end{aligned}
$$

Но указанные условия Вирасоро в точности совпадают с условиями Вирасоро матричной модели Концевича (3.14)! Таким образом, получено каноническое преобразование переменных исходной матричной модели (2.37), которое превращает эту модель в две копии ММК, взятые при различных временах. Учитывая инвариантность вакуума относительно этих преобразований, получим для модели (2.37) следующее утверждение.

Теорема 5. Статистические суммы ММКП (2.37) и $M M K$ (2.17) связаны точныцм соотношением

$$
e^{\mathcal{F}_{\mathrm{KP}}\left(\left\{T_{2 n}^{ \pm}\right\}\right)}=e^{C(\alpha N)} e^{-\mathcal{A}} e^{\mathcal{F}_{\mathrm{K}}\left(\left\{\xi_{n}^{+}\right\}\right)+\mathcal{F}_{\mathrm{K}}\left(\left\{\xi_{n}^{-}\right\}\right)} \cdot \mathbf{1},
$$

где $\xi_{n}^{ \pm}= \pm T_{2 n}^{ \pm}$и $\mathcal{A}$ - квадратичный дифференциальный оператор по $\partial / \partial \xi^{ \pm}$:

$$
\begin{aligned}
\mathcal{A}= & \sum_{m, n=0}^{\infty} \frac{B_{2(n+m+1)}}{4(n+m+1)} \frac{1}{(2 n+1) !(2 m+1) !} \times \\
& \times\left\{\frac{\partial}{\partial \xi_{m}^{+}} \frac{\partial}{\partial \xi_{n}^{+}}+\frac{\partial}{\partial \xi_{m}^{-}} \frac{\partial}{\partial \xi_{n}^{-}}-2\left(2^{2(n+m+1)}-1\right) \frac{\partial}{\partial \xi_{m}^{+}} \frac{\partial}{\partial \xi_{n}^{-}}\right\}- \\
& -\sum_{n=2}^{\infty} \alpha N \frac{2^{2 n-1}}{(2 n+1) !}\left(\frac{\partial}{\partial \xi_{n}^{-}}+\frac{\partial}{\partial \xi_{n}^{+}}\right) .
\end{aligned}
$$

Здесь $C(\alpha N)$ - функция, зависящая лишь от $\alpha N$, которая обеспечивает условие $\mathcal{F}_{\mathrm{KP}}\left(\left\{T_{2 n}^{ \pm}\right\}\right)=0$ при $T_{2 n}^{ \pm} \equiv 0 ; B_{2 k}-$ числа Бернулли.

Еще один интересный сюжет - это интерпретация матричных моделей с точки зрения КТП. Некоторые сведения о такой связи для ММКП содержатся в работе [104]. 
Благодарности. Автор благодарен своим соавторам Я. Амбьерну, А. В. Забродину, К. Л. Зарембо, Ш. Кристиансен, Ю. М. Макеенко и К. В. Паламарчуку. Работа была поддержана грантами INTAS № 99-1782 и РФФИ № 99-01-00151 и № 00-15-96046.

\section{Список литературы}

[1] A. M. Polyakov. Phys. Lett. B. 1981. V. 103. P. 207; P. 211.

[2] J. Polchinsky. Nucl. Phys. B. 1989. V. 324. P. 123.

[3] V. Knizhnik, A. Polyakov, A. Zamolodchikov. Mod. Phys. Lett. A. 1988. V. 3. P. 819.

[4] F. David. Mod. Phys. Lett. A. 1988. V. 3. P. 1651.

[5] J. Distler, H. Kawai. Nucl. Phys. B. 1989. V. 312. P. 509.

[6] J. Ambjørn, B. Durhuus, J. Fröhlich, P. Orland. Nucl. Phys. B. 1986. V. 270. P. 457.

[7] E. Witten. Nucl. Phys. B. 1990. V. 340. P. 281.

[8] E. Witten. On the Kontsevich model and other models of two-dimensional gravity. In: Proc. of the XX Int. Conf. on Diff. Geom. Methods in Theor. Phys. (New York, 1991). V. 1. Eds. S. Catto and A. Rocha. River Edge, N.J.: World Scientific, 1992. P. 176.

[9] М. Л. Концевич. Функц. анализ и его прилож. 1991. Т. 25. С. 50; M. L. Kontsevich. Commun. Math. Phys. 1992. V. 147. P. 1.

[10] G.'t Hooft. Nucl. Phys. B. 1974. V. 72. P. 461.

[11] P. Di Francesco, P. Ginsparg, J. Zinn-Justin. Phys. Rep. 1995. V. 254. P. 1; hep-th/9306153.

[12] А.Ю. Морозов. УФН. 1994. Т. 164. С. 3.

[13] M. L. Mehta. Random Matrices. New York: Academic Press, 1991.

[14] J. Ambjørn, B. Durhuus, T. Jonsson. Quantum Geometry. A Statistical Field Theory Approach. Cambridge: Cambridge Univ. Press, 1997.

[15] E. Brézin, C. Itzykson, G. Parisi, J.-B. Zuber. Commun. Math. Phys. 1978. V. 59. P. 35.

[16] E. Brézin, V. Kazakov. Phys. Lett. B. 1990. V. 236. P. 144.

[17] M. Douglas, S. Shenker. Nucl. Phys. B. 1990. V. 335. P. 635.

[18] D. J. Gross, A. Migdal. Phys. Rev. Lett. 1990. V. 64. P. 127.

[19] А. Д. Миронов. $\tau$-Функции и матричные модели. Дисс. докт. ф.-м. н. М.: ФИАН, 1997.

[20] S. Kharchev. Am. Math. Soc. Transl. (2). 1999. V. 191. P. 119.

[21] E. Witten. Surv. Diff. Geom. 1991. V. 1. P. 243.

[22] V. Kazakov, I. Kostov, N. Nekrasov. Nucl. Phys. B. 1999. V. 557. P. 413; hep-th/9810035.

[23] T. Banks, W. Fischler, S. H. Shenker, L. Susskind. Phys. Rev. D. 1997. V. 55. P. 5112; hep-th/9610043.

[24] N. Ishibashi, H. Kawai, Y. Kitazawa, A. Tsuchiya. Nucl. Phys. B. 1997. V. 498. P. 467; hep-th/9612115.

[25] А. Б. Замолодчиков. Письма в ЖЭТФ. 1987. Т. 46. С. 129.

[26] А. Ю. Морозов. УФН. 1992. Т. 162. № 8. С. 83.

[27] В. Книжник. УФН. 1989. Т. 159. № 3. С. 401.

[28] S. Saito. Phys. Rev. Lett. 1987. V. 59. P. 1798.

[29] S. Saito. Phys. Rev. D. 1987. V. 36. P. 1819.

[30] A. Morozov. Phys. Lett. B. 1987. V. 196. P. 325.

[31] M. Fukuma, H. Kawai, R. Nakayama. Int. J. Mod. Phys. A. 1991. V. 6. P. 1385.

[32] A. Mironov, A. Morozov. Phys. Lett. B. 1990. V. 252. P. 47.

[33] J. Ambjørn, J. Jurkiewicz, Yu. Makeenko. Phys. Lett. B. 1990. V. 251. P. 517.

[34] A. Gerasimov, A. Marshakov, A. Mironov, A. Morozov, A. Orlov. Nucl. Phys. B. 1991. V. 357. P. 565.

[35] H. Itoyama, Y. Matsuo. Phys. Lett. B. 1991. V. 255. P. 202.

[36] H.S. La. Commun. Math. Phys. 1991. V. 140. P. 569.

[37] Yu. Makeenko, A. Marshakov, A. Mironov, A. Morozov. Nucl. Phys. B. 1991. V. 356. P. 574. 
[38] C. Itzykson, J.-B. Zuber. Int. J. Mod. Phys. A. 1992. V. 7. P. 5661.

[39] R. Dijkgraaf, E. Verlinde, H. Verlinde. Nucl. Phys. B. 1991. V. 348. P. 435.

[40] D. J. Gross, M. J. Newman. Phys. Lett. B. 1991. V. 266. P. 291; Nucl. Phys. B. 1992. V. 380. P. 168.

[41] A. Marshakov, A. Mironov, A. Morozov. Phys. Lett. B. 1992. V. 274. P. 280.

[42] R. Dijkgraaf, E. Verlinde, H. Verlinde. Nucl. Phys. B. 1991. V. 352. P. 59.

[43] E. Witten. Nucl. Phys. B. 1992. V. 371. P. 191.

[44] S. Kharchev, A. Marshakov, A. Mironov, A. Morozov, A. Zabrodin. Phys. Lett. B. 1992. V. 275. P. 311; Nucl. Phys. B. 1992. V. 380. P. 181.

[45] E. Brézin, D. Gross. Phys. Lett. B. 1980. V. 97. P. 120.

[46] R. C. Brower, M. Nauenberg. Nucl. Phys. B. 1981. V. 180[FS2]. P. 221.

[47] Yu. Makeenko, G. Semenoff. Mod. Phys. Lett. A. 1991. V. 6. P. 3455.

[48] Yu. Makeenko. Mod. Phys. Lett. (Brief Reviews) A. 1991. V. 6. P. 1901.

[49] В. А. Воеводский, Г. Б. Шабат. ДАН СССР. 1989. Т. 304. № 2. С. 265.

[50] Г. Бельй. Изв. АН СССР. Сер. матем. 1980. Т. 14. № 2. С. 247.

[51] P. B. Cohen, C. Itzykson, J. Wolfart. Preprint Saclay T93/048; Commun. Math. Phys. 1994. V. 163. P. 605.

[52] L. Chekhov. Geom. and Phys. 1993. V. 12. P. 153.

[53] J. Ambjørn, L. Chekhov, C. F. Kristjansen, Yu. Makeenko. Nucl. Phys. B. 1993. V. 404. P. 127.

[54] L. Chekhov, Yu. Makeenko. Mod. Phys. Lett. A. 1992. V. 7. P. 1223.

[55] J. Harer, D. Zagier. Invent. Math. 1986. V. 85. P. 457.

[56] R. C. Penner. Commun. Math. Phys. 1987. V. 113. P. 299; J. Diff. Geom. 1988. V. 27. P. 35 .

[57] J. Distler, C. Vafa. Mod. Phys. Lett. A. 1991. V. 6. P. 259.

[58] L. Chekhov, A. Zabrodin. Mod. Phys. Lett. A. 1991. V. 6. P. 3143.

[59] Л. О. Чехов. Матричные модели и геометрия пространства модулей. Дисс. докт. ф.-м. н. М: МИАН, 2000.

[60] J. Ambjørn, L. Chekhov, Yu. Makeenko. Phys. Lett. B. 1992. V. 282. P. 341.

[61] D. Gross, E. Witten. Phys. Rev. D. 1980. V. 21. P. 446.

[62] L. Chekhov, Yu. Makeenko. Phys. Lett. B. 1992. V. 278. P. 271.

[63] A. Fayyazuddin, Y. Makeenko, P. Olesen, D. J. Smith, K. Zarembo. Nucl. Phys. B. 1997. V. 499. P. 159; hep-th/9703038.

[64] C.F. Kristiansen, P. Olesen. Phys. Lett. B. 1997. V. 405. P. 45; hep-th/9704017.

[65] A. Mironov, A. Morozov, G. W. Semenoff. Int. J. Mod. Phys. A. 1996. V. 11. P. 5031.

[66] L. Chekhov, K. Zarembo. Mod. Phys. Lett. A. 1997. V. 12. P. 2331.

[67] J. Ambjørn, L. Chekhov. The NBI matrix model of IIB superstring. Preprint JHEP 9812:007, 1998; hep-th/9805212.

[68] L. Chekhov, K. Palamarchuk. Mod. Phys. Lett. A. 1999. V. 14. № 32. P. 2229.

[69] I. Kostov. Mod. Phys. Lett. A. 1989. V. 4. P. 217.

[70] B. Eynard, J. Zinn-Justin. Nucl. Phys. B. 1992. V. 386. P. 558.

[71] B. Eynard, C. F. Kristjansen. Nucl. Phys. B. 1995. V. 455. P. 577; hep-th $/ 9506193$.

[72] L. Chekhov, C. Kristjansen. Nucl. Phys. B. 1996. V. 479[FS]. P. 683.

[73] K. Ueno, K. Takasaki. Toda lattice hierarchy. In: Group Representation and Systems of Differential Equations (Tokyo, 1982). Adv. Stud. Pure Math. V. 4. Ed. K. Okamoto. Amsterdam: North-Holland, 1984. P. 1.

[74] K. Strebel. Quadratic Differentials. Berlin: Springer, 1984.

[75] J. Harer. The cohomology of the moduli space of curves. In: Lect. Notes in Math. V. 1337. Theory of Moduli (Montecatini Terme, 1985). Ed. S. Sernesi. Berlin: Springer, 1988. P. 138.

[76] P. Deligne, M. Mumford. Publ. IHES. 1969. V. 45. P. 75. 
[77] D. Mumford. Towards an enumerative geometry of the moduli space of curves. In: Arithmetic and Geometry. V. II (Progr. Math. V. 36). Eds. M. Artin, J. Tate. Boston, MA: Birkhäuser, 1983. P. 271.

[78] R. Dijkgraaf. Intersection theory, integrable hierarchies and topological field theory. In: New Symmetry Principles in Quantum Field Theory. Proc. of NATO Adv. Study Inst. Held in Cargese, July 16-27 1991. Eds. J. Frölich, G. 't Hooft, A. Jaffe, G. Mack, P. K. Mitter, R. Stora. New York: Plenum, 1992. P. 95; hep-th/9201003.

[79] V. Kazakov. Mod. Phys. Lett. A. 1989. V. 4. P. 2125.

[80] A. A. Migdal. Phys. Rep. 1983. V. 102. P. 199.

[81] S. Dalley, C. Johnson, T. Morris. Nucl. Phys. B. 1992. V. 368. P. 625; P. 655.

[82] К. Л. Зарембо, Л. О. Чехов. ТМФ. 1992. Т. 93. № 2. С. 354.

[83] F. David. Mod. Phys. Lett. A. 1990. V. 5. P. 1019.

[84] J. Ambjørn, C.F. Kristjansen, Yu. Makeenko. Mod. Phys. Lett. A. 1992. V. 7. P. 3187; hep-th/9207020.

[85] L. Alvarez-Gaumé, J. L. Mañes. Mod. Phys. Lett. A. 1991. V. 6. P. 2039; L. Alvarez-Gaumé, H. Itoyama, J. L. Mañes, A. Zadra. Int. J. Mod. Phys. A. 1992. V. 7. P. 5337; hep-th/9112018.

[86] J. C. Plefka. Nucl. Phys. B. 1995. V. 448. P. 355; hep-th/9504089.

[87] J. Ambjørn, Yu. Makeenko. Mod. Phys. Lett. A. 1990. V. 5. P. 1753.

[88] C. Bachas, P. M.S. Petropoulos. Phys. Lett. B. 1990. V. 247. P. 363.

[89] Yu. Makeenko. Loop equations and Virasoro constraints in matrix models. In: Proc. XXVth Intl. Symp. on the Theory of Elementary Particles. Ed. H. J. Kaiser. Gösen, 1991. P. 275.

[90] L. Motl. Proposals on nonperturbative superstring interactions. hep-th/9701025.

[91] S. Sethi, L. Susskind. Phys. Lett. B. 1997. V. 400. P. 265; hep-th/9702101.

[92] T. Banks, N. Seiberg. Nucl. Phys. B. 1997. V. 497. P. 41; hep-th/9702187.

[93] R. Dijkgraaf, E. Verlinde, H. Verlinde. Nucl. Phys. B. 1997. V. 500. P. 43; hep-th/9703030.

[94] М. Грин, Дж. Шварц, Е. Виттен. Ведение в теорию суперструн. М.: Мир, 1990.

[95] J. Polchinsky. Superstrings. V.1,2. Cambridge: Cambridge Univ. Press, 1998.

[96] T. Yoneya. Progr. Theor. Phys. 1997. V. 97. P. 949; hep-th/9703078.

[97] К. Л. Зарембо, Ю. М. Макеенко. УФН. 1998. Т. 168. № 1. С. 3.

[98] L. Paniak, N. Weiss. J. Math. Phys. 1995. V. 36. P. 2512; hep-th/9501037.

[99] R. Dijkgraaf, G. Moore, R. Plesser. Nucl. Phys. B. 1993. V. 394. P. 356.

[100] C. Imbimbo, S. Mukhi. Nucl. Phys. B. 1995. V. 449. P. 553.

[101] R. C. Brower, P. Rossi, C.-I. Tan. Phys. Rev. D. 1981. V. 23. P. 942.

[102] Yu. Makeenko. Phys. Lett. B. 1993. V. 314. P. 197.

[103] Yu. Makeenko. Int. J. Mod. Phys. A. 1995. V. 10. P. 2615.

[104] L. Chekhov. Acta Appl. Math. 1997. V. 48. P. 33. 